\title{
GUTs and exceptional branes in F-theory - I
}

\section{Citation}

Beasley, Chris, Jonathan J Heckman, and Cumrun Vafa. 2009. “GUTs and Exceptional Branes in F-Theory - I." Journal of High Energy Physics 2009 (1): 058-058. https:// doi.org/10.1088/1126-6708/2009/01/058.

\section{Permanent link}

http://nrs.harvard.edu/urn-3:HUL.InstRepos:41385025

\section{Terms of Use}

This article was downloaded from Harvard University's DASH repository, and is made available under the terms and conditions applicable to Open Access Policy Articles, as set forth at http:// nrs.harvard.edu/urn-3:HUL.InstRepos:dash.current.terms-of-use\#OAP

\section{Share Your Story}

The Harvard community has made this article openly available.

Please share how this access benefits you. Submit a story.

\section{Accessibility}


arXiv:0802.3391

\title{
GUTs and Exceptional Branes in F-theory - I
}

\author{
Chris Beasley*, Jonathan J. Heckman ${ }^{\dagger}$ and Cumrun Vafa \\ Jefferson Physical Laboratory, Harvard University, Cambridge, MA 02138, USA
}

\begin{abstract}
Motivated by potential phenomenological applications, we develop the necessary tools for building GUT models in F-theory. This approach is quite flexible because the local geometrical properties of singularities in F-theory compactifications encode the physical content of the theory. In particular, we show how geometry determines the gauge group, matter content and Yukawa couplings of a given model. It turns out that these features are beautifully captured by a four-dimensional topologically twisted $\mathcal{N}=4$ theory which has been coupled to a surface defect theory on which chiral matter can propagate. From the vantagepoint of the four-dimensional topological theory, these defects are surface operators. Specific intersection points of these defects lead to Yukawa couplings. We also find that the unfolding of the singularity in the F-theory geometry precisely matches to properties of the topological theory with a defect.
\end{abstract}

February, 2008

\footnotetext{
*e-mail: beasley@physics.harvard .edu

$\dagger$ e-mail: jheckman@fas.harvard.edu

$\ddagger$ e-mail: vafa@physics.harvard.edu
} 


\section{Contents}

1 Introduction 3

2 General Overview $\quad 9$

3 Partially Twisted Gauge Theory on a Seven-Brane 12

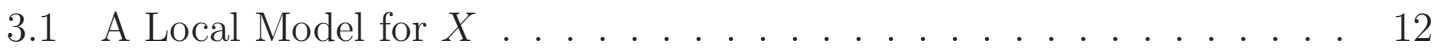

3.2 Twisting on $S \ldots \ldots \ldots \ldots \ldots \ldots \ldots \ldots$

3.3 The Four-Dimensional Effective Theory . . . . . . . . . . . . . . . . 25

3.3 .1 BPS Equations . . . . . . . . . . . . . . 27

3.3.2 Bulk Spectrum of Massless Particles . . . . . . . . . . . . . . 29

3.3.3 Bulk Yukawa Couplings . . . . . . . . . . . . . . . . . 33

3.4 Bulk Toy Models . . . . . . . . . . . . . . . . . . . . . . . . . 34

4 Intersecting Seven-Branes in F-Theory $\quad 37$

4.1 Colliding Singularities and Intersecting Seven-Branes . . . . . . . . . 39

4.2 Topological Field Theory on a Defect . . . . . . . . . . . . . . . . . . 45

4.2.1 Topological Twist on $\Sigma$. . . . . . . . . . . . . . 46

4.2.2 On the Supersymmetric Defect Action . . . . . . . . . . . . . 49

4.2.3 The Defect as a Cosmic String . . . . . . . . . . . . . . . . 54

4.3 Unfolding Singularities via Surface Operators . . . . . . . . . . . . . 59

4.3.1 Brane Recombination . . . . . . . . . . . . . . . . . 60

4.3.2 General Unfolding . . . . . . . . . . . . . . . . . . 64

4.4 Chiral Matter and Yukawa Couplings from $\Sigma \ldots . . . . . . . . .668$

4.4.1 Massless Spectrum . . . . . . . . . . . . . . . . . . 69

4.4.2 More Yukawa Couplings . . . . . . . . . . . . . . . . 70

4.4.3 A Refined Toy Model . . . . . . . . . . . . . . . . . . . . 71

5 Multiple Intersections and More Yukawa Couplings $\quad 74$

5.1 Enhancement to A-Type Singularities . . . . . . . . . . . . . . . . 76 
5.2 Cubic Couplings from Codimension Two . . . . . . . . . . . 80

5.3 Local Cubic Couplings for E-Type Singularities . . . . . . . . . . 81

6 A Final Toy Model $\quad 83$

$\begin{array}{lll}7 & \text { Conclusions } & 87\end{array}$

A Review of Hirzebruch and del Pezzo Surfaces 89

B On-Shell Twisted Supersymmetries of the $\begin{array}{ll}\text { Eight-Dimensional Theory } & 91\end{array}$

C Partially Twisted Action of the Seven-Brane Theory 93

D Partially Twisted Action of the $\begin{array}{ll}\text { Six-Dimensional Defect Theory } & 100\end{array}$

$\begin{array}{ll}\text { E A Vanishing Theorem } & 106\end{array}$

F Explicit Deformations of an $E_{7}$ Singularity 112

G Higher Exotic Singularities $\quad 114$ 


\section{Introduction}

String theory appears to provide a large number of consistent vacua which can accomodate the observed features of the Universe. Given this perhaps embarassment of riches, it is natural to ask whether the deeper understanding of non-perturbative features of string theory obtained in the post-duality era provides some degree of uniqueness or at least some novel concrete predictions for upcoming experiments.

There are encouraging signs that string theory naturally includes many of the qualitative features of the Standard Model such as classical unitary gauge groups and bifundamental chiral matter. Indeed, these are ubiquitous features of D-brane realizations of gauge theories. Reviews of the vast literature of models which attempt to realize the Standard Model via D-branes may be found in $[1,2,3,4]$.

Independent of its connection to string theory, a compelling motivation for low energy supersymmetry is that the particle content of the MSSM improves the unification of the gauge coupling constants observed in the Standard Model. But because the a priori independent volumes of cycles wrapped by D-branes control the values of the gauge coupling constants, D-brane constructions of Standard Model-like vacua tend to obscure this fact. Both gauge coupling unification as well as the matter content of the Standard Model hint at the presence of a unified gauge group structure at high energies. ${ }^{1}$ In fact, this unification naturally suggests the presence of an exceptional gauge group looming in the background. Indeed, there is a natural sequence of $E$-group embeddings ${ }^{2}$ depicted in figure 1 which give the Standard Model gauge group and matter structure in an elegant manner:

$$
E_{3} \times U(1) \subset E_{4} \subset E_{5} \subset E_{6} \subset \ldots
$$

where $E_{3}=S U(3) \times S U(2)$ denotes the non-abelian gauge group of the Standard Model, $E_{4}=S U(5)$ and $E_{5}=S O(10)$. Some early field theory realizations of this paradigm may be found in $[6,7,8]$.

\footnotetext{
${ }^{1}$ This is not to say that the unification of gauge couplings in GUT-like models cannot be realized in D-brane constructions. See [5] for one early realization of a D-brane GUT.

${ }^{2}$ Note that even though in four dimensions only $E_{6}$ and lower rank exceptional groups can contain chiral matter, in the context of higher dimensional theories coming from string theory, all of these groups can have chiral matter. In this sense string theory completes the link between GUT theories and all exceptional groups by bringing the higher dimensions into play.
} 

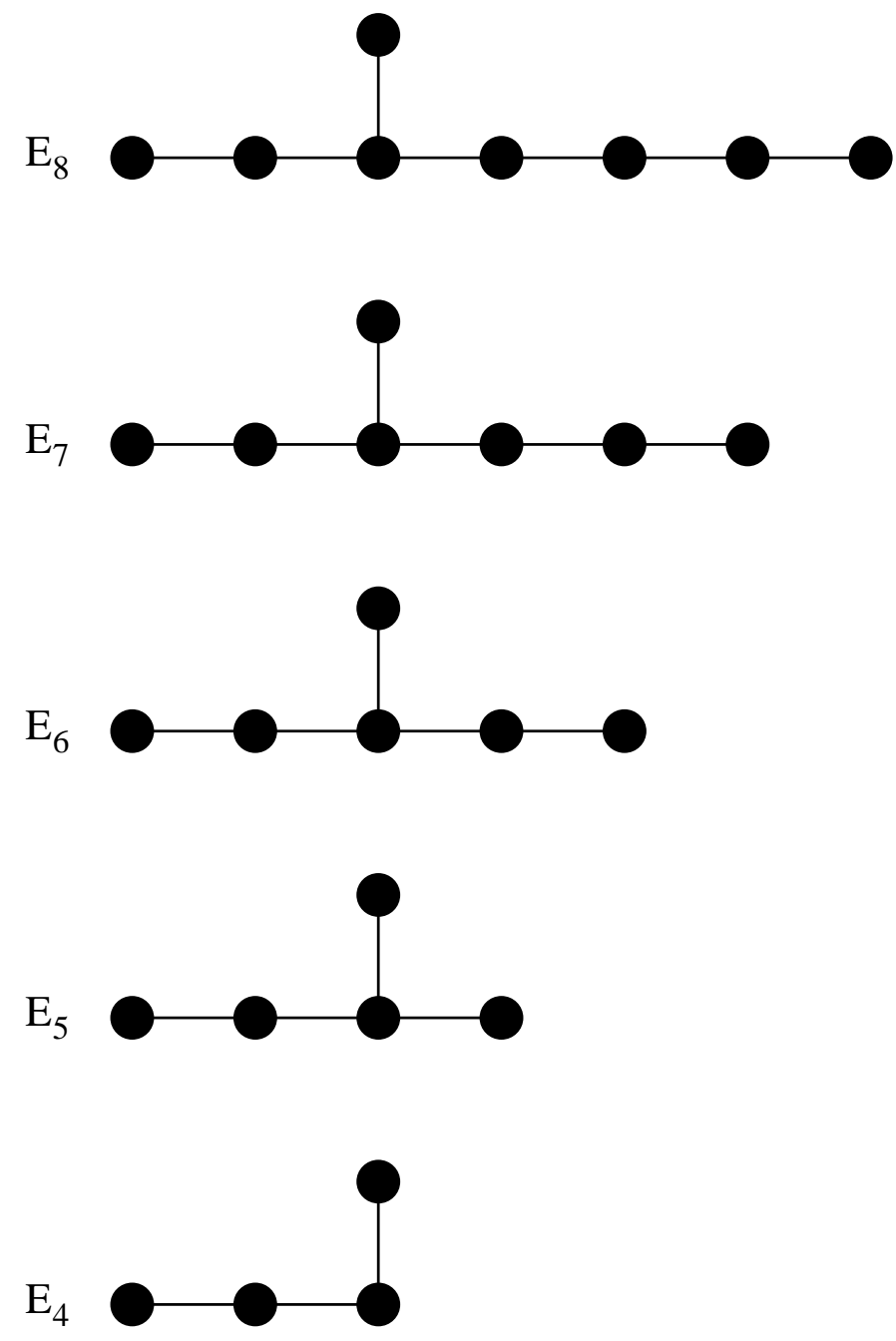

○

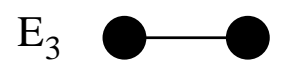

Figure 1: Dynkin diagrams for the $E$-series of Lie Groups. Starting from $E_{8}$, deleting the rightmost node of each successive diagram produces the next entry. The entry $E_{3}=S U(3) \times S U(2)$ is the non-abelian gauge group of the Standard Model. 
Moreover, packaging the field content of the Standard Model into the appropriate GUT multiplet is not always possible in D-brane realizations. Indeed, although the classical groups appear in this sequence of embeddings, only one and two index tensor representations of the gauge groups can appear in D-brane constructions. In particular, for $S O(10)$ GUTs, the matter content of the Standard Model organizes into the $\mathbf{1 6}$ spinor representation. This representation is conspicuously absent from perturbative D-brane setups. Further issues pertaining to D-brane realizations of GUT models are in discussed in [9].

From this perspective, we interpret the unification of the gauge couplings in the minimal supersymmetric extension of the Standard Model as evidence that instead of the infinitely complex variety of classical groups and matter which can appear in string theory, the list of relevant simple gauge groups is limited to the finite number of exceptional gauge groups and their subgroups. ${ }^{3}$ This is a vast simplification!

In this paper we take as given that the gauge groups unify into a GUT and further that the matter content of the Standard Model descends naturally from the representation content of an exceptional gauge group. ${ }^{4}$ For this reason, we now focus on how exceptional gauge groups arise in string theory. Perhaps the most obvious answer is the $E_{8} \times E_{8}$ heterotic string, as was already exploited more than two decades ago [11]. Nevertheless, as reviewed in [12], the simplest approach in this direction does not quite succeed because the perturbative heterotic $E_{8} \times E_{8}$ string compactified on an isotropic Calabi-Yau threefold makes an (incorrect) prediction for the relation between $M_{G U T}$ and $M_{p l}$ :

$$
\frac{M_{G U T}^{2}}{M_{p l}^{2}} \gtrsim \alpha_{G U T}^{4 / 3} .
$$

Witten has shown in [12] that the above bound can be significantly weakened in the strongly coupled regime of the heterotic $E_{8} \times E_{8}$ string described by M-theory compactified on $S^{1} / \mathbb{Z}_{2}$. In the Hořava-Witten description, each $E_{8}$ gauge group

\footnotetext{
${ }^{3}$ Alternatively, even if one views gauge coupling unification as an accident, non-trivial qualitative criteria such as the existence of a self-similar duality cascade structure can emerge from properties natural to D-branes in the large $N$ limit of the MSSM or some minimal extension thereof [10].

${ }^{4}$ By this we do not mean that we will restrict our model building efforts to four-dimensional $E_{6}$ GUTs. Indeed, as we shall argue below, many of the necessary features of even more conventional GUTs require the presence of an $E_{6,7,8}$-type gauge group which may be broken by the geometry of the F-theory compactification.
} 
factor confines to a ten-dimensional "end of the Universe" boundary of the elevendimensional M-theory compactification [13]. As shown in [12], separating the two boundaries far away from one another significantly weakens the above bound. At a pragmatic level this allows us to focus on one of the $E_{8}$ boundary walls as leading to the observed gauge symmetries of the Standard Model.

But many properties of the $E_{8}$ wall are mysterious. In particular, the argument for the choice of the $E_{8}$ gauge group is based primarily on anomaly considerations. This fact presents a hindrance towards a more detailed description of the properties of the matter content and their interactions.

Further insight is possible for exceptional gauge groups which are realized by the geometry of exceptional singularities in string theory. For example, real codimension four gauge theories defined by type IIA in six dimensions or M-theory in seven dimensions can provide a geometric understanding of exceptional gauge groups when the internal compactified directions are local singularities of the type:

$$
\mathbb{C}^{2} / \Gamma
$$

where $\Gamma$ is one of the three exceptional subgroups of $S U(2)$, leading to $E_{6,7,8}$ gauge symmetries. Similarly, compactifications of F-theory on such a singularity descend to eight-dimensional gauge theories. This eight-dimensional theory is interpreted as the worldvolume of a non-perturbative seven-brane in a type IIB compactification. ${ }^{5}$ As opposed to the $E_{8}$ wall of Hořava-Witten theory, in the geometric approach there are a number of analytic tools available for describing the unfolding of geometric singularities.

An $\mathcal{N}=1$ supersymmetric GUT theory with exceptional gauge group derived from a geometric singularity can arise either from M-theory compactified on a sevendimensional manifold of $G_{2}$ holonomy [15] or from F-theory compactified on an elliptic Calabi-Yau fourfold [16]. In the $G_{2}$ case, the absence of holomorphic structure limits the analytic control over detailed properties of the geometry. By contrast, in the Calabi-Yau fourfold case, the complex/Kähler geometry allows a more powerful array of techniques. For this reason, compactifications of F-theory on Calabi-Yau fourfolds preserving $\mathcal{N}=1$ supersymmetry in four dimensions have been extensively

\footnotetext{
${ }^{5}$ In fact, the Hořava-Witten $E_{8}$ wall can also be related to such singularities upon further compactification [14].
} 
studied. A foundational example of this work is [17]. To the best of our knowledge, however, no systematic study of the connection between F-theory and GUTs has been undertaken (see, however, [18] for some progress in this direction). Some work in this direction has been done in connection with models with a heterotic dual. Even in these examples, though, a direct analysis from the vantagepoint of F-theory has not been given yet. However, as we explain below, we do not wish to assume that a given model has a well-defined heterotic dual. Our aim in this work and the followup paper [19] is to take a first step in filling this gap. We note that independent work on extracting the chiral matter content directly from F-theory and matching these results to heterotic duals has recently appeared in [20].

At a foundational level, there is another reason to study models constructed from $E$-type singularities in F-theory in their own right, as opposed to appealing to a potential heterotic dual. Recall that F-theory compactifications with a heterotic dual derive from the basic duality between compactifications of F-theory on an elliptic $K 3$ and its heterotic dual on a $T^{2}$. Extending this duality fiberwise over a complex surface $S$, we achieve a duality between heterotic strings compactified on CalabiYau threefolds elliptically fibered over $S$ and F-theory compactified on a Calabi-Yau fourfold given by an elliptic $K 3$-fibration over $S$. Geometrically, the condition that an elliptic fibration of $S$ yields a Calabi-Yau threefold requires that $S$ be of Fano type. Said differently, there exist a large class of heterotic and F-theory compactifications which may not possess a dual description. In order to maintain maximal flexibility for future model building applications, we shall therefore not limit our considerations to models with a well-defined heterotic dual. ${ }^{6}$

As mentioned previously, gauge groups in F-theory arise from codimension one singularities in the base which are in turn identified with the worldvolume of some seven-branes. In order to maintain a finite gauge coupling constant in the fourdimensional effective theory on $\mathbb{R}^{3,1}$, we assume that the seven-brane wraps a compact complex surface $S$ of real dimension four. Turning on a supersymmetric gauge field configuration on $S$ in some subgroup $H_{S} \subset G_{S}$ breaks the gauge group $G_{S}$ to the commutant of $H_{S}$ in $G_{S}$. This provides an economical way to break the exceptional gauge group to gauge symmetries closer to the MSSM. Moreover, when this supersymmetric gauge field configuration has a non-trivial overall $U(1)$ factor,

\footnotetext{
${ }^{6}$ In particular, in Section 7 we speculate on one possible model building application when $S$ is not a Fano variety.
} 
the resulting spectrum can contain chiral matter originating from the zero modes of fields propagating in the bulk of $S$.

Along a codimension two subspace of the threefold base defined by a Riemann surface $\Sigma \subset S$, the rank of the singularity type can increase. As discussed in [21, 22], this leads to matter living on $\Sigma$. The Riemann surface $\Sigma$ can be viewed as the intersection locus of two singularities fibered over two different complex surfaces $S$ and $S^{\prime}$. From the perspective of the threefold base, $S^{\prime}$ can be viewed as the locus of another seven-brane which may be non-compact. The fields localized on $\Sigma$ are a direct extension of the bi-fundamental fields obtained from the intersection of D-branes to the more general case of colliding singularities. A non-trivial background gauge field configuration on $\Sigma$ can also induce a four-dimensional chiral matter spectrum.

The fields of the MSSM interact via cubic couplings. In the present class of models, Yukawa couplings among chiral matter come about in three different ways. The first type correspond to couplings between three bulk fields on $S$. When $S$ is a Hirzebruch or del Pezzo surface, these couplings all vanish. The second type of coupling comes from the interaction between two fields living on $\Sigma$ and one living on $S(\Sigma \Sigma S)$. The last type of Yukawa coupling occurs when the zero mode wave functions for fields localized on three such $\Sigma$ 's intersect at a point $(\Sigma \Sigma \Sigma)$. This type of coupling turns out to be generic in the case of exceptional singularities. ${ }^{7}$

It turns out that there is a beautiful interplay between the topological field theories which dictate the matter content coming from $S$ and $\Sigma$. The relevant degrees of freedom living on $S$ are captured by an $\mathcal{N}=4$ topologically twisted theory of the type studied in [24]. Furthermore, the matter fields coming from $\Sigma$ are welldescribed by another topological field theory defined on $\Sigma$ which naturally couples to the bulk theory on $S$ as a defect theory. The coupling of these two topological theories leads to sources for some of the fields in the bulk theory on $S$ when the fields on $\Sigma$ develop a vev. This turns out to nicely correspond to the unfolding of the singularity in F-theory.

The primary aim of the present paper is to flesh out these ideas and to set the groundwork for potential applications. Although we shall present some examples of semi-realistic models, more phenomenologically viable constructions of GUTs from

\footnotetext{
${ }^{7}$ In some very special situations studied in [23], a further enhancement in the singularity may not correspond to an $A D E$ type singularity. We discuss some of the physics of this case in Appendix G.
} 
F-theory will appear in the followup paper [19].

The rest of this paper is organized as follows. In Section 2 we describe the basic setup. In Section 3 we describe the simplest F-theory geometry with exceptional singularities localized on an isolated seven-brane. Furthermore, we introduce the eight-dimensional partially twisted topological theory corresponding to the worldvolume of a general seven-brane and show that the geometric unfolding of the singularity exactly matches to degrees of freedom in the gauge theory. We also consider the possibility of turning on supersymmetric background gauge fields on the seven-brane and analyze the resulting (chiral) matter and existence (or absence) of Yukawa couplings among them. In Section 4 we introduce the partially twisted theory describing a more general class of compactifications in which seven-branes intersect along Riemann surfaces in $S$. In this same section, we also discuss how four-dimensional chiral matter can arise on such intersections and compute the Yukawa couplings between pairs of chiral matter fields living on a Riemann surface and bulk gauge fields in $S$. Even in this more general class of models, we observe a harmonious match between gauge theory and geometric degrees of freedom. In Section 5 we study subloci of real codimension four in $S$ along which the singularity type enhances to even higher rank. We find that for certain geometries, such points signal the presence of additional Yukawa couplings among chiral matter fields with wave functions localized on Riemann surfaces. In Section 6 we present a toy model which incorporates many of the ingredients developed in previous Sections. Section 7 presents our conclusions. Additional background and more technical material is included in the Appendices.

\section{General Overview}

In this section we present an overview of the types of models we shall treat. Our setup is locally given by the worldvolume of a seven-brane of generalized $A D E$-type in a compactification on a Calabi-Yau fourfold. Letting $S$ denote a Kähler manifold of complex dimension two which is wrapped by the seven-brane, the resulting local model will reduce to an $\mathcal{N}=1$ supersymmetric theory in four dimensions. In order to work in the limit in which gauge dynamics decouple from gravity, we sometimes restrict our attention to geometries where $S$ can shrink to zero size inside a general threefold base. This is also natural from the viewpoint of the strongly coupled limit of the heterotic $E_{8} \times E_{8}$ string in that the bound of (1.2) can be evaded. In addition, in 
this limit there are no additional massless scalar fields corresponding to the motion of $S$ inside the threefold base of the F-theory compactification. For all of these reasons, we shall sometimes require that the anti-canonical bundle of the surface $S$ is ample so that such a contraction is possible. This amounts to the condition that $S$ is a del Pezzo surface.

As will be discussed in greater detail in later Sections, there are in general two ways in which chiral matter can arise from such a theory. The first corresponds to turning on a gauge field configuration on $S$ with non-trivial first Chern class. Given a field which transforms in a representation of the structure group for the holomorphic gauge bundle on $S$, the number of net generations will be given by an index computation.

Another source of matter originates from six-dimensional chiral fields localized along Riemann surfaces in $S$. In many cases this can be interpreted as the intersection locus of distinct seven-branes. This chirality can be preserved in four dimensions when a suitable background gauge field configuration has been turned on along the Riemann surface. To see how this comes about, recall that an F-theory compactification will generically contain three types of singularities. Over the locus given by the holomorphic surface $S$, the corresponding singularity of $A D E$ type will give rise to a gauge group which we denote by $G_{S}$. This singularity may collide with another singularity with gauge group $G_{S^{\prime}}$ supported on a non-compact complex surface $S^{\prime}$. See figure 2 for a depiction of the intersecting seven-brane locus in the case where $S$ is compact and $S^{\prime}$ is non-compact. Over the Riemann surface $\Sigma$ defined by the intersection of $S$ and $S^{\prime}$, the singularity type enhances to $G_{\Sigma}$ such that:

$$
G_{\Sigma} \supset G_{S} \times G_{S^{\prime}}
$$

For the case of $S U(N+M) \supset S U(N) \times S U(M)$, this corresponds to intersecting D7-branes and the matter localized at the intersection transforms in the $(N, \bar{M})$. More generally, the six-dimensional chiral matter localized on $\Sigma$ transforms under the analogous projection of the adjoint of $G_{\Sigma}$ to $G_{S}$ as in [22] for breaking $G_{\Sigma}$ to a subgroup with some number of $U(1)$ factors. When the bulk gauge field on $\Sigma$ has non-trivial first Chern class, the resulting spectrum in four dimensions can be chiral. The net number of generations localized on $\Sigma$ is given by an index computation on the Riemann surface for fields charged under the given combination of background $U(1)$ gauge fields. 


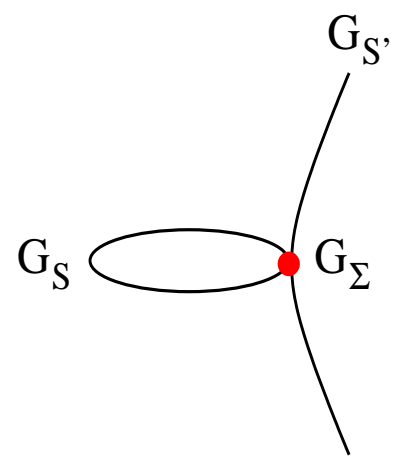

Figure 2: Depiction of intersecting seven-branes wrapping a compact surface $S$ with gauge group $G_{S}$ and a non-compact surface $S^{\prime}$ with non-dynamical gauge group $G_{S^{\prime}}$. In the threefold base of the F-theory compactification, the intersection locus of $S$ and $S^{\prime}$ is a Riemann surface where the singularity type enhances further to $G_{\Sigma} \supset G_{S} \times G_{S^{\prime}}$.

We now explain the origin of Yukawa couplings in this setup. There are a priori three ways in which non-trivial interaction terms can arise. The first possibility corresponds to interactions between three bulk fields of the eight-dimensional theory. As we will argue in Section 3, such interaction terms are identically zero when $S$ is either a Hirzebruch or del Pezzo surface. The second type of interaction term originates from the coupling between a single bulk field on $S$ with two matter fields localized along a Riemann surface $\Sigma$ in $S$. The final type of interaction originates from the triple overlap at a single point in $S$ of matter fields localized along three matter curves. We find that in many cases of interest, such triple overlaps are a generic feature of F-theory compactifications.

One may also wonder if there are instanton corrections to the Yukawa couplings. In F-theory the natural source for such instantons are wrapped Euclidean D3-branes [25], which in the present case means wrapping D3-branes on $S$. Note, however, that this is nothing but the usual contribution from gauge theory instantons. In the regime of interest for GUT models where $1 / g_{Y M}^{2}>>1$, such contributions lead to very small corrections on the order of $\exp \left(-a / g_{Y M}^{2}\right)$ for some $O(1)$ constants $a$, so for the purposes of this paper we shall ignore these effects. 


\section{Partially Twisted Gauge Theory on a Seven- Brane}

As a warmup for the rest of the paper, in this Section we determine the fourdimensional effective theory for a class of particularly simple F-theory compactifications which preserve only $\mathcal{N}=1$ supersymmetry in four dimensions. Specifically, in Section 3.1 we consider local F-theory geometries which describe a small neighborhood of a seven-brane with worldvolume $\mathbb{R}^{3,1} \times S$, where $S$ is a compact Kähler surface and the worldvolume gauge group $G_{S}$ is of arbitrary $A D E$-type.

In Section 3.2 we argue that the dynamics of the low-energy degrees of freedom in F-theory are captured by a partially twisted version of the maximally supersymmetric Yang-Mills theory on $\mathbb{R}^{3,1} \times S$. We also present evidence that the partially twisted Yang-Mills theory correctly describes the moduli of the given background in F-theory.

In Section 3.3 we determine some basic properties of the four-dimensional effective theory for this special class of F-theory models. In particular, we present the BPS equations of motions for the eight-dimensional fields in the partially twisted YangMills theory and subsequently study supersymmetric vacua in the presence of a non-trivial background gauge field configuration on $S$. We find that while many vacua support a chiral matter spectrum in four dimensions, when $S$ is a Hirzebruch or del Pezzo surface, all Yukawa couplings identically vanish.

\subsection{A Local Model for $X$}

To set our notation, we consider F-theory [16, 26, 27] on a background of the form $\mathbb{R}^{3,1} \times X$, where $X$ is a Calabi-Yau fourfold. By assumption, the fourfold $X$ fibers elliptically with a section over a complex threefold $B$,

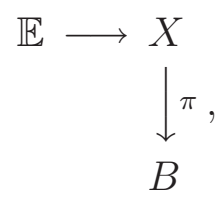


and in general, the elliptic fiber $\mathbb{E}$ of $X$ degenerates over a locus $\Delta$ of complex codimension one in $B$,

$$
\Delta \subset B
$$

Physically, $\Delta$ encodes the location of various seven-branes in $B$, and the nature of the singularities in $\mathbb{E}$ over $\Delta$ determines the worldvolume gauge group on each seven-brane. From the perspective of the present paper, what is extremely interesting about F-theory is that with the appropriate singularity in $\mathbb{E}$, seven-branes wrapping $\Delta$ can carry a worldvolume gauge group of arbitrary ${ }^{8} A D E$-type. While the gauge groups $S U(n+1)$ or $S O(2 n)$ can be realized using D7-branes and (in the latter case) orientifold planes, a seven-brane with worldvolume gauge group $E_{6,7,8}$ is a rather mysterious object that cannot be described perturbatively in string theory. We shall refer to such seven-branes as "exceptional".

In addition to seven-branes, a general F-theory background may also contain spacetime filling D3-branes which sit at points in $B$, as well as supersymmetric fluxes associated to either the bulk supergravity fields on $B$ or to the worldvolume gauge fields on $\Delta$. However, for reasons to become clear momentarily, only the worldvolume fluxes on $\Delta$ will play a role in the F-theory models we study.

F-theory on $X$ typically represents a strongly-coupled background of string theory, and to make progress in even our low-energy analysis, we make two simplifying assumptions. First, we focus attention throughout on local, non-compact models which describe only a small neighborhood of $\Delta$ inside $B$, with neither D3-branes nor bulk fluxes present. ${ }^{9}$ With this assumption, gravity decouples in four dimensions, and modulo exotic possibilities such as that reviewed in Appendix G, we expect to obtain an $\mathcal{N}=1$ supersymmetric gauge theory on $\mathbb{R}^{3,1}$ which captures the effective dynamics of the light worldvolume degrees-of-freedom living on $\Delta$. This gauge theory is the analogue for seven-branes in F-theory of the well-known quiver gauge theories which describe D-branes at singularities in perturbative compactifications of type IIB string theory on Calabi-Yau threefolds.

Even in local models for $B$, the geometry of the seven-brane configuration repre-

\footnotetext{
${ }^{8}$ In the presence of monodromies, even non-simply-laced gauge groups are possible, though we will not exploit this possibility in the present paper.

${ }^{9}$ The D3-branes on $\Delta$ are included in our construction as they would correspond to internal point-like instantons for gauge fields on $\Delta$, which is a special case of what we will study in this paper.
} 
sented by $\Delta$ can be quite complicated. For instance, $\Delta$ might be reducible and hence appear as a union of several components associated to loci of colliding singularities in the elliptic fibration over $B$. As our second simplifying assumption, we suppose that $\Delta$ consists only of an irreducible, smooth, compact, complex surface $S$ embedded in $B$. So in this Section, we study the worldvolume theory on a single seven-brane which wraps $\mathbb{R}^{3,1} \times S$ in F-theory on $X$. Later in Sections 4 and 5, we extend our analysis to the case that $\Delta$ is reducible and multiple seven-branes intersect in $B$.

Before we proceed to a fairly detailed analysis of the worldvolume gauge theory living on a seven-brane of arbitrary $A D E$-type wrapping $\mathbb{R}^{3,1} \times S$, let us first present a local model for the Calabi-Yau fourfold $X$ which describes F-theory in the background of such a brane. Since we are already working with a local model for $B$, we will also work with a local model for $X$. We thus take $X$ to be a local elliptic $K 3$-fibration over $S$ of the form

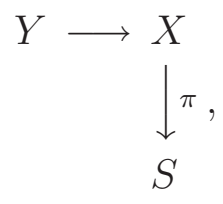

where we model the local elliptically-fibered $K 3$-surface $Y$ on a hypersurface in $\mathbb{C}^{3}$ with an isolated $A D E$ singularity at the origin.

Not suprisingly, the $A D E$ singularities play a prominent role throughout the paper. In terms of coordinates $(x, y, z)$ on $\mathbb{C}^{3}$, we recall that the $A D E$ singularities can be presented canonically as below.

\begin{tabular}{|l|l|}
\hline$A_{n}$ & $y^{2}=x^{2}+z^{n+1}$ \\
$D_{n}$ & $y^{2}=x^{2} z+z^{n-1}$ \\
$E_{6}$ & $y^{2}=x^{3}+z^{4}$ \\
$E_{7}$ & $y^{2}=x^{3}+x z^{3}$ \\
$E_{8}$ & $y^{2}=x^{3}+z^{5}$ \\
\hline
\end{tabular}

In the following work we shall sometimes rescale these coordinates by overall numerical coefficients in order to more easily compare our results with the unfolding of singularities in [28]. 
If $X$ is to be Calabi-Yau, then the hypersurface $Y$ must fiber appropriately over $S$. To describe the fibering, we promote the local coordinates $(x, y, z)$ in (3.4) to transform as sections of a rank three bundle $V$ over $S$ which is given as a sum of tensor powers of the canonical bundle $K_{S}$,

$$
V=K_{S}^{a} \oplus K_{S}^{b} \oplus K_{S}^{c} .
$$

Here $(a, b, c)$ are three integers associated to $(x, y, z)$ which we must determine.

The integers $(a, b, c)$ necessarily satisfy two conditions for the simple local model of $X$ to exist. First, in order that the defining equations for $Y$ in (3.4) make sense, the individual terms in each equation must transform as sections of the same line bundle over $S$. This yields the homogeneity conditions:

$$
\begin{array}{|l|l|}
\hline A_{n} & 2 b=2 a=(n+1) c \\
D_{n} & 2 b=2 a+c=(n-1) c \\
E_{6} & 2 b=3 a=4 c \\
E_{7} & 2 b=3 a=a+3 c \\
E_{8} & 2 b=3 a=5 c \\
\hline
\end{array}
$$

Second, the Calabi-Yau condition on $X$ implies that the holomorphic two-form $(1 / y) d x \wedge d z$ on $Y$ transforms over $S$ as a section of $K_{S}$. Thus $(a, b, c)$ also satisfy

$$
a-b+c=1 .
$$

Together, the equations in (3.6) and (3.7) admit the following unique solutions.

$$
\begin{array}{|l|l|l|l|}
\hline A_{n} & a=\frac{n+1}{2} & b=\frac{n+1}{2} & c=1 \\
D_{n} & a=n-2 & b=n-1 & c=2 \\
E_{6} & a=4 & b=6 & c=3 \\
E_{7} & a=6 & b=9 & c=4 \\
E_{8} & a=10 & b=15 & c=6 \\
\hline
\end{array}
$$

For the cases other than $A_{n}$ with $n$ even, $(a, b, c)$ in (3.8) are integers, so the local model for $X$ which describes a seven-brane of $A D E$-type wrapping $S$ clearly exists. 
To treat the case of $A_{n}$ with $n$ even, for which we see that $a$ and $b$ in (3.8) are only half-integral, we make an elementary change of variables to realize the $A_{n}$ singularity as $x y=z^{n+1}$. Homogeneity of the latter equation implies that $a+b=(n+1) c$, and the Calabi-Yau condition on $X$ now fixes $c=1$. Hence in this form, a local model for $X$ with $A_{n}$ singularity over $S$ exists for arbitrary integers $a$ and $b$ which satisfy $a+b=n+1$.

Now, one interesting fact about the E-type singularities in (3.4) is that these singularities appear automatically in the Weierstrass form:

$$
y^{2}=x^{3}+f(z) x+g(z)
$$

Here $x$ and $y$ can be interpreted as affine coordinates parameterizing the elliptic fiber $\mathbb{E}$ of $X$, and $f(z)$ and $g(z)$ are determined as simple monomials in $z$ by (3.4). In particular, since $x$ and $y$ parameterize $\mathbb{E}$, the coordinate $z$ must parameterize the normal direction to $S$ inside the non-compact threefold $B$. Thus for $E_{6,7,8}$, the base $B$ is given by the total spaces of the respective line bundles $K_{S}^{3}, K_{S}^{4}$, and $K_{S}^{6}$ over $S$. The particular numerology that occurs here will later be quite significant for the worldvolume description of the exceptional seven-brane.

For $A$ - and $D$-type singularities, the coordinate $z$ is similarly distinguished as parameterizing the normal direction to $S$ inside $B$. This assertion can be checked directly by analyzing global Weierstrass models for the elliptic singularities. Alternatively, in the $A_{n}$ case, one can simply note that according to (3.8), $z$ transforms as a section of the canonical bundle $K_{S}$. Hence, if $z$ parameterizes the normal direction to $S$ inside $B$, the threefold $B$ is itself Calabi-Yau, as one expects for seven-branes which carry a perturbative gauge group of type $A_{n}$.

Additionally, in the case of a $D_{n}$ singularity, $B$ is the total space of $K_{S}^{2}$ over $S$. The total space of $K_{S}^{2}$ can be considered as the quotient of the local Calabi-Yau threefold associated to $K_{S}$ by a $\mathbb{Z}_{2}$ involution of the fiber. This involution fixes $S$ as the zero-section of $K_{S}$ and acts freely elsewhere, so we naturally obtain a local orientifold geometry, as expected for seven-branes which carry a gauge group of type $D_{n}$. 


\subsection{Twisting on $S$}

To determine the effective worldvolume description of the seven-brane wrapping $\mathbb{R}^{3,1} \times S$, let us begin with the trivial case that $S=\mathbb{C}^{2}$. In this case, F-theory reduced to $\mathbb{R}^{7,1}=\mathbb{R}^{3,1} \times \mathbb{C}^{2}$ on the hypersurface $Y$ with an isolated $A D E$ singularity is described at low energies by the maximally supersymmetric Yang-Mills theory with gauge group $G_{S}$ of corresponding $A D E$-type. ${ }^{10}$

If we now regard $\mathbb{C}^{2}$ as a local patch of $S$, the standard adiabatic argument suggests that $F$-theory on $X$ is still described at low energies by eight-dimensional Yang-Mills theory on $\mathbb{R}^{3,1} \times S$. However, in order to preserve $\mathcal{N}=1$ supersymmetry, this Yang-Mills theory must be topologically twisted on $S$. By the end of the Section, we will present some very suggestive evidence that the partially twisted Yang-Mills theory on $\mathbb{R}^{3,1} \times S$ does describe the light degrees of freedom of F-theory on $X$.

The idea of studying F-theory on $X$ by means of the topological gauge theory living on the worldvolume of the seven-brane wrapping $\mathbb{R}^{3,1} \times S$ is not new. This idea, including certain elaborations we consider later, was sketched over ten years ago in one of the foundational papers [17] on $\mathcal{N}=1$ F-theory compactifications. However, the details behind some of the ideas in [17] seem never to have been fleshed out, and doing so is one of our goals here.

Actually, to refer to the twisted Yang-Mills theory on $\mathbb{R}^{3,1} \times S$ as "topological" is a bit of a misnomer. In order to twist the eight-dimensional Yang-Mills theory on $S$, we will have to use the fact that $S$ is not a generic Riemannian four-manifold but rather, through the embedding of $S$ into $B$ and hence $X$, carries an induced Kähler structure. As we explain later, the observables of primary interest which determine the effective superpotential in four dimensions will be insensitive to the particular Kähler metric on $S$. On the other hand, these observables will certainly depend on the complex structure of $S$.

For supersymmetric gauge theories in dimensions three and four, many qualitatively distinct possiblities for twisting exist. However, the possibilities for twisting supersymmetric gauge theories in higher dimensions are much more restricted. Given that $S$ is Kähler, the maximally supersymmetric Yang-Mills theory in eight dimensions admits a unique twist on $\mathbb{R}^{3,1} \times S$ which preserves $\mathcal{N}=1$ supersymmetry in four dimensions. As a result, once we argue that F-theory on $X$ is described by

\footnotetext{
${ }^{10}$ Note that because $Y$ is non-compact, gravity decouples in eight dimensions.
} 
a twisted Yang-Mills theory on $\mathbb{R}^{3,1} \times S$, we have no choice about which twist to consider.

The procedure of twisting the maximally supersymmetric Yang-Mills theory on $\mathbb{R}^{3,1} \times S$ is entirely standard, but since this gauge theory and its later elaborations provide our basic tool for studying $\mathrm{F}$-theory on $X$, we now discuss the twist in some detail.

We start with the maximally supersymmetric Yang-Mills theory in ten dimensions on $\mathbb{R}^{9,1}$. In ten dimensions, the super Yang-Mills multiplet consists of a gauge field and an adjoint-valued fermion which transforms under $S O(9,1)$ in the positivechirality spinor representation $\mathbf{1 6}_{+}$. This theory preserves sixteen supersymmetries, which transform in the representation $\mathbf{1 6} \mathbf{6}_{+}$.

Under reduction to $\mathbb{R}^{7,1}$, the Yang-Mills multiplet decomposes into an eightdimensional gauge field which we denote by $A$, two real scalar fields $\Phi_{8}$ and $\Phi_{9}$, and two fermions $\Psi_{ \pm}$. Each of the scalars and fermions transforms in the adjoint representation of the gauge group. For later use, we now introduce the following complex-linear combinations of $\Phi_{8}$ and $\Phi_{9}$,

$$
\varphi=\Phi_{8}+i \Phi_{9}, \quad \bar{\varphi}=\Phi_{8}-i \Phi_{9} .
$$

The eight-dimensional Yang-Mills theory preserves $S O(7,1) \times U(1)_{R}$ as a global symmetry, under which the fermions $\Psi_{ \pm}$transform as respective summands in the reducible representation

$$
\mathbf{1 6}_{+} \longmapsto\left(\mathrm{S}_{+},+\frac{1}{2}\right) \oplus\left(\mathrm{S}_{-},-\frac{1}{2}\right)
$$

Here $\mathbf{S}_{ \pm}$denote the positive and negative chirality spinor representations of $S O(7,1)$. Also, the complex scalar fields $\varphi$ and $\bar{\varphi}$ transform trivially under $S O(7,1)$ and with charges $\mp 1$ under $U(1)_{R}$.

To twist the gauge theory on $\mathbb{R}^{7,1}=\mathbb{R}^{3,1} \times \mathbb{C}^{2}$, we further reduce the global symmetry group from $S O(7,1) \times U(1)_{R}$ to

$$
S O(3,1) \times S O(4) \times U(1)_{R}
$$

Under (3.12), both the fermions $\Psi_{ \pm}$and the corresponding supersymmetry generators 
$\epsilon_{ \pm}$transform as

$$
\begin{aligned}
& \left(\mathrm{S}_{+},+\frac{1}{2}\right) \longmapsto\left[(2, \mathbf{1}),(\mathbf{2}, \mathbf{1}),+\frac{1}{2}\right] \oplus\left[(\mathbf{1}, \mathbf{2}),(\mathbf{1}, \mathbf{2}),+\frac{1}{2}\right] \\
& \left(\mathrm{S}_{-},-\frac{1}{2}\right) \longmapsto\left[(\mathbf{2}, \mathbf{1}),(\mathbf{1}, \mathbf{2}),-\frac{1}{2}\right] \oplus\left[(\mathbf{1}, \mathbf{2}),(\mathbf{2}, \mathbf{1}),-\frac{1}{2}\right] .
\end{aligned}
$$

Here we make use of the standard local isomorphism $S O(4) \cong S U(2) \times S U(2)$, and similarly for $S O(3,1)$, to describe the representation content in $(3.13)$. Thus, $(\mathbf{2}, \mathbf{1})$ describes the left handed chiral spinor of either $S O(4)$ or $S O(3,1)$, and $(\mathbf{1}, \mathbf{2})$ describes the right handed anti-chiral spinor.

If $S$ were an arbitrary Riemannian four-manifold, we would now specify the twist on $S$ by an embedding of the global $U(1)_{R}$ symmetry into $S O(4)$, identified with the structure group of the tangent bundle of $S$. However, at this point we make use of the fact that $S$ is Kähler so that the structure group of the tangent bundle of $S$ actually reduces from $S O(4)$ to $U(2)$. Hence the possible twists on $S$ are specified by embeddings of $U(1)_{R}$ into the smaller group $U(2)$.

Up to isomorphism, a unique topological twist is possible, under which $U(1)_{R}$ is embedded into the central $U(1)$ subgroup of $U(2)$. To present the twist, we let $R$ be the generator of $U(1)_{R}$. Similarly, we let $J$ be the generator of the central $U(1)$ subgroup in $U(2)$. We normalize $J$ so that under the reduction from $S O(4)$ to $U(2)$, the chiral and anti-chiral spinor representations of $S O(4)$ decompose as

$$
(2,1) \rightarrow \mathbf{2}_{0}, \quad(\mathbf{1}, \mathbf{2}) \rightarrow \mathbf{1}_{+1} \oplus \mathbf{1}_{-1},
$$

where the subscripts in (3.14) denote the $U(1)$ charges under the central generator $J$.

Comparing (3.14) to (3.13), we see that in order to obtain four scalar supercharges on $S$ which descend to the standard $\mathcal{N}=1$ supersymmetry generators $Q_{\alpha}$ and $\bar{Q}_{\dot{\alpha}}$ on $\mathbb{R}^{3,1}$, the new central $U(1)$ generator $J_{\text {top }}$ in the twisted $U(2)$ must be given by one of the following linear combinations of the original generators $J$ and $R$,

$$
J_{\text {top }}=J \pm 2 R .
$$

As one can check, either sign in (3.15) leads to an isomorphic twist, so we take 
$J_{\text {top }}=J+2 R$ without loss of generality. This choice leads to somewhat more natural conventions regarding holomorphy in the twisted Yang-Mills theory on $S$.

In the twisted theory, the fermions $\Psi_{ \pm}$and supersymmetry generators $\epsilon_{ \pm}$now transform under $S O(3,1) \times U(2)$ as

$$
\begin{aligned}
& {\left[(\mathbf{2}, \mathbf{1}) \otimes \mathbf{2}_{+1}\right] \oplus\left[(\mathbf{1}, \mathbf{2}) \otimes\left(\mathbf{1}_{+2} \oplus \mathbf{1}_{0}\right)\right],} \\
& {\left[(\mathbf{1}, \mathbf{2}) \otimes \mathbf{2}_{-1}\right] \oplus\left[(\mathbf{2}, \mathbf{1}) \otimes\left(\mathbf{1}_{0} \oplus \mathbf{1}_{-2}\right)\right] .}
\end{aligned}
$$

Here the subscripts in (3.16) denote the charges under the central $U(1)$ generator $J_{\text {top }}$ in $U(2)$. In particular, from the representations $(\mathbf{1}, \mathbf{2}) \otimes \mathbf{1}_{0}$ and $(\mathbf{2}, \mathbf{1}) \otimes \mathbf{1}_{0}$ appearing in (3.16), we see that the twisted theory on $S$ possesses four scalar supercharges $\left(Q_{\alpha}, \bar{Q}_{\dot{\alpha}}\right)$, associated to the fact that F-theory on $X$ preserves $\mathcal{N}=1$ supersymmetry in four dimensions.

By the same token, the complex scalar fields $\varphi$ and $\bar{\varphi}$ now transform under $S O(3,1) \times U(2)$ as:

$$
(\mathbf{1}, \mathbf{1}) \otimes \mathbf{1}_{\mp 2}
$$

To interpret the twisted fermions in (3.16) geometrically, we fix conventions under which the central $U(1)$ in $U(2)$ acts on vectors in the holomorphic tangent bundle $T S$ with charge +1 and dually on covectors in the holomorphic cotangent bundle $\Omega_{S}^{1}$ with charge -1 . The twisted fermions then appear as forms of holomorphic/antiholomorphic type $(p, 0)$ and $(0, p)$ on $S$ for $p=0,1,2$. Corresponding to (3.16), we denote the twisted fermions by:

$$
\begin{array}{ll}
\bar{\eta}_{\dot{\alpha}} & \text { section of } \operatorname{ad}(P), \\
\psi_{\alpha}=\psi_{\alpha} \bar{m} d \bar{s}^{\bar{m}} & \text { section of } \bar{\Omega}_{S}^{1} \otimes \operatorname{ad}(P), \\
\bar{\chi}_{\dot{\alpha}}=\bar{\chi}_{\dot{\alpha} \overline{m n}} d \bar{s}^{\bar{m}} \wedge d \bar{s}^{\bar{n}} & \text { section of } \bar{\Omega}_{S}^{2} \otimes \operatorname{ad}(P),
\end{array}
$$

and

$$
\begin{array}{ll}
\eta_{\alpha} & \text { section of } \operatorname{ad}(P), \\
\bar{\psi}_{\dot{\alpha}}=\bar{\psi}_{\dot{\alpha} m} d s^{m} & \text { section of } \Omega_{S}^{1} \otimes \operatorname{ad}(P), \\
\chi_{\alpha}=\chi_{\alpha m n} d s^{m} \wedge d s^{n} & \text { section of } \Omega_{S}^{2} \otimes \operatorname{ad}(P) .
\end{array}
$$

Here, $s^{m}$ and $\bar{s}^{\bar{m}}$ are local holomorphic and anti-holomorphic coordinates on $S$ which we use to indicate the transformations of the twisted fermions as differential forms on 
$S$. As standard, we also use $\alpha$ and $\dot{\alpha}$ for $\alpha, \dot{\alpha}=1,2$ to indicate the transformations of the twisted fermions as either chiral or anti-chiral spinors on $\mathbb{R}^{3,1}$. Finally, ad $(P)$ is the adjoint bundle associated to a fixed principal $G_{S}$-bundle $P$ over $S$. Here we anticipate the possibility of turning on a background instanton on $S$ associated to the choice of a topologically non-trivial $G_{S}$-bundle $P$.

From (3.17), we also see that the twisted complex scalar $\varphi$ now transforms on $S$ as a section of $\Omega_{S}^{2} \otimes \operatorname{ad}(P)$,

$$
\varphi=\varphi_{m n} d s^{m} \wedge d s^{n},
$$

and the conjugate scalar $\bar{\varphi}$ transforms as a section of $\bar{\Omega}_{S}^{2} \otimes \operatorname{ad}(P)$,

$$
\bar{\varphi}=\bar{\varphi}_{\overline{m n}} d \bar{s}^{\bar{m}} \wedge d \bar{s}^{\bar{n}}
$$

Equivalently, $\varphi$ transforms as a section of $K_{S} \otimes \operatorname{ad}(P)$, and $\bar{\varphi}$ transforms as a section of $\bar{K}_{S} \otimes \operatorname{ad}(P)$.

\section{A Small Paradox, and Its Resolution}

Before delving further into the details of the twisted Yang-Mills theory on $S$, let us discuss the basic physical interpretation of the twist. As we will explain, the transformation of $\varphi$ as a section of $K_{S} \otimes \operatorname{ad}(P)$ presents a small paradox, whose resolution proves to illuminate a basic feature of exceptional seven-branes in F-theory.

In the case that the seven-brane is a D7-brane in a perturbative compactification of type IIB string theory, the transformation of the worldvolume scalar $\varphi$ as a section of $K_{S} \otimes \operatorname{ad}(P)$ is very easy to understand. In that situation, $B$ is a Calabi-Yau threefold, and by the standard adjunction formula, the canonical bundle $K_{S}$ of $S$ is isomorphic to the holomorphic normal bundle $N_{S / B}$ of $S$ inside $B$. Hence the twist of $\varphi$ amounts to the geometric statement that $\varphi$ describes normal motion of the D7-brane wrapping $S$ inside $B$ [29].

More generally, if $B$ is not Calabi-Yau, the adjunction formula provides an isomorphism

$$
K_{S}=\left.K_{B}\right|_{S} \otimes N_{S / B}
$$

where $\left.K_{B}\right|_{S}$ denotes the restriction of the canonical bundle of $B$ to $S$. For a sevenbrane of $D$ - or $E$-type, the threefold $B$ is not Calabi-Yau and $K_{B}$ is non-trivial, so we see from (3.22) that the normal bundle $N_{S / B}$ is generally not isomorphic to the canonical bundle $K_{S}$ in those cases. 
On the other hand, the topological twist of Yang-Mills theory on $S$ uniquely fixes $\varphi$ to transform as a section of $K_{S} \otimes \operatorname{ad}(P)$ for a seven-brane of arbitrary $A D E$ type. So if our geometric intuition for a D7-brane in a perturbative Calabi-Yau compactification is taken at face value, the fact that $\varphi$ generally transforms as a section of $K_{S} \otimes \operatorname{ad}(P)$ as opposed to $N_{S / B} \otimes \operatorname{ad}(P)$ on the exceptional seven-brane presents a small paradox.

The resolution of this paradox turns out to be very instructive and provides immediate evidence that the topologically twisted Yang-Mills theory on $\mathbb{R}^{3,1} \times S$ serves as a valid worldvolume description for exceptional seven-branes in F-theory.

To explain why $\varphi$ generally transforms as a section of $K_{S} \otimes \operatorname{ad}(P)$ as opposed to $N_{S / B} \otimes \operatorname{ad}(P)$, we recall that the moduli for the positions of seven-branes in $F$-theory are encoded by complex structure moduli of the Calabi-Yau fourfold $X$ itself, since these moduli determine the location in $B$ of the discriminant locus $\Delta$ on which the seven-branes wrap. Thus to understand how $\varphi$ describes the position of seven-branes in $B$, we must really ask how $\varphi$ can be used to deform the complex structure of $X$. In our local model, $X$ is a fibration of the $A D E$ hypersurface $Y$ over $S$, so we effectively want to consider how an expectation value for $\varphi$ translates into a deformation of the canonical equations for the $A D E$-singularities in (3.4).

As a simple example, let us consider the case that $Y$ describes an $A_{n}$ singularity over $S$, for which the seven-brane is an ordinary D7-brane. The general deformation of the $A_{n}$ singularity in (3.4) can be parameterized by $n+1$ complex variables $\left(t_{1}, \ldots, t_{n+1}\right)$ subject to the single constraint $t_{1}+\cdots+t_{n+1}=0$, in terms of which the deformation is given by

$$
\begin{aligned}
y^{2} & =x^{2}+\prod_{j=1}^{n+1}\left(z+t_{j}\right) \\
& =x^{2}+z^{n+1}+\sum_{k=2}^{n+1} s_{k}\left(t_{1}, \ldots, t_{n+1}\right) z^{n+1-k}
\end{aligned}
$$

Here $s_{k}$ is the elementary symmetric polynomial which is homogeneous of degree $k$ in the variables $t_{1}, \ldots, t_{n+1}$.

Not coincidentally, the parameters $\left(t_{1}, \ldots, t_{n+1}\right)$ can also be interpreted as coordinates on the Cartan subalgebra of the group $G_{S}=S U(n+1)$, in which form we identify them with the eigenvalues of the adjoint-valued scalar field $\varphi$. Equivalently, 
we identify the degree $k$ symmetric polynomial $s_{k}$ with the degree $k$ Casimir of $\varphi$,

$$
\begin{aligned}
s_{2}\left(t_{1}, \ldots, t_{n+1}\right) & =-\frac{1}{2} \operatorname{Tr}\left(\varphi^{2}\right), \\
& \vdots \\
s_{n+1}\left(t_{1}, \ldots, t_{n+1}\right) & =\operatorname{det}(\varphi) .
\end{aligned}
$$

Now, if the deformation in (3.23) is to make sense when $z$ transforms as a section of $K_{S}$, the degree $k$ polynomial $s_{k}\left(t_{1}, \cdots, t_{n+1}\right)$ must transform as a section of $K_{S}^{k}$, simply so that the deformed equation in (3.23) remains homogeneous as an equation on sections of $K_{S}^{(n+1)}$. Under the identification in (3.24), this requirement is equivalent to the condition that $\varphi$ transform as a section of $K_{S} \otimes \operatorname{ad}(P)$. Thus, as we certainly expect, the automatic twisting of $\varphi$ in the Yang-Mills theory on $\mathbb{R}^{3,1} \times S$ is consistent with the non-trivial fibration of $Y$ over $S$ which defines $X$.

For the $D$ - and $E$-type singularities, precisely the same geometric reasoning implies that $\varphi$ still transforms as a section of $K_{S} \otimes \operatorname{ad}(P)$. Just as for the $A_{n}$ singularities, we recall [28] that the deformations of the general $D$ - or $E$-type singularities are parameterized by coordinates $t_{j}$ for $j=1, \ldots, r$ on the Cartan subalgebra of the group $G_{S}$, where $r=\operatorname{rk}\left(G_{S}\right)$ is the rank of $G_{S}$. In terms of $\left(t_{1}, \cdots, t_{r}\right)$, the deformations of the $A D E$ singularities in (3.4) can be presented as in the table below.

\begin{tabular}{|l|l|}
\hline$A_{n}$ & $y^{2}=x^{2}+z^{n+1}+\sum_{k=2}^{n+1} \alpha_{k} z^{n+1-k}$ \\
\hline$D_{n}$ & $y^{2}=-x^{2} z+z^{n-1}+\sum_{k=1}^{n-1} \delta_{2 k} z^{n-k-1}-2 \gamma_{n} x$ \\
\hline$E_{6}$ & $y^{2}=x^{3}+\frac{z^{4}}{4}+\varepsilon_{2} x z^{2}+\varepsilon_{5} x z+\varepsilon_{6} z^{2}+\varepsilon_{8} x+\varepsilon_{9} z+\varepsilon_{12}$ \\
\hline$E_{7}$ & $y^{2}=-x^{3}+16 x z^{3}+\varepsilon_{2} x^{2} z+\varepsilon_{6} x^{2}+\varepsilon_{8} x z+$ \\
& $+\varepsilon_{10} z^{3}+\varepsilon_{12} x+\varepsilon_{14} z+\varepsilon_{18}$ \\
\hline$E_{8}$ & $y^{2}=x^{3}-z^{5}+\varepsilon_{2} x z^{3}+\varepsilon_{8} x z^{2}+\varepsilon_{12} z^{3}+\varepsilon_{14} x z+$ \\
& $+\varepsilon_{18} z^{2}+\varepsilon_{20} x+\varepsilon_{24} z+\varepsilon_{30}$ \\
\hline
\end{tabular}

Up to shifts of coordinates by an overall constant, the particular choices of minus signs and numerical coefficients in the table above have been chosen in order to conform with the presentation of the singularities of table 3 in [28] so that for example, 
$\alpha_{k}=s_{k} \cdot{ }^{11}$ For ease of comparison, we have retained the conventions of [28] for labelling the deformation parameters of the $D$ - and $E$ - type singularities.

By analogy with the role of the symmetric polynomials $s_{k}$ in (3.23), each of the $\delta_{2 k}, \gamma_{k}$, and $\varepsilon_{k}$ appearing in (3.25) is one of the fundamental invariant polynomials of degree $k$ on the Cartan subalgebra of $G_{S}$. Specifically, for the case of a $D_{n}$ singularity, the polynomials $\delta_{2 k}$ are invariant polynomials of degree $2 k$ on the Cartan subalgebra of $S O(2 n)$, given in terms of the elementary symmetric polynomials by $\delta_{2 k}=s_{k}\left(t_{1}^{2}, \ldots, t_{n}^{2}\right)$, and $\gamma_{n}=t_{1} t_{2} \cdots t_{n}$ is the invariant polynomial of degree $n$ which represents the Pfaffian of $S O(2 n)$. Equivalently in terms of Casimirs of $S O(2 n)$

$$
\begin{aligned}
\delta_{2}\left(t_{1}, \cdots t_{n}\right) & =-\frac{1}{2} \operatorname{Tr}\left(\varphi^{2}\right), \\
& \vdots \\
\gamma_{n}\left(t_{1}, \cdots, t_{n}\right) & =\operatorname{Pf}(\varphi) .
\end{aligned}
$$

For the $E$-type singularities, each $\varepsilon_{k}$ is an invariant polynomial of degree $k$ in the coordinates $t_{i}$ which parameterize the Cartan subalgebra of $E_{6,7,8}$. The invariant polynomials for $E_{6,7,8}$ are notoriously complicated, but thankfully at the moment we only require knowledge of their total degrees, indicated by the subscripts in (3.25).

In order for the deformations in (3.25) to make sense geometrically when the affine coordinates $(x, y, z)$ transform in the specific powers of $K_{S}$ given in (3.8), each invariant polynomial $\delta_{2 k}, \gamma_{k}$, and $\varepsilon_{k}$ must transform in the tensor power of $K_{S}$ whose degree matches the degree of the polynomial in $\left(t_{1}, \ldots, t_{r}\right)$. For instance, in the case of the $E_{8}$ singularity, each term appearing in the deformed equation in (3.25) must transform as a section of $K_{S}^{30}$, since $y^{2}, x^{3}$, and $z^{5}$ all transform as sections of $K_{S}^{30}$ according to (3.8). As one can easily check, this condition implies that the invariant polynomial $\varepsilon_{k}$ of degree $k$ on the Cartan subalgebra of $E_{8}$ must transform as a section of the corresponding tensor power $K_{S}^{k}$, where $k$ runs over

$$
(2,8,12,14,18,20,24,30) \text {. }
$$

\footnotetext{
${ }^{11}$ Here we deviate slightly from [28] by writing $y^{2}=x^{2}+z^{n+1}$ as opposed to $x y=z^{n+1}$. This distinction is unimportant, though, because the deformations of the singularity are all independent of $x$ and $y$. The presentation given here proves convenient for providing a uniform treatment of the Calabi-Yau fourfold condition for all singularities.
} 
Once again, we physically interpret the coordinates $\left(t_{1}, \ldots, t_{r}\right)$ on the Cartan subalgebra of $G_{S}$ as the eigenvalues of the adjoint-valued scalar field $\varphi$. Because

each degree $k$ Casimir of $\varphi$ then transforms as a section of $K_{S}^{k}$, consistency requires that $\varphi$ itself must transform as a section of $K_{S} \otimes \operatorname{ad}(P)$, just as we found from the twisted Yang-Mills theory on $S$.

This simple observation is our first check that the degrees of freedom of the twisted gauge theory on $\mathbb{R}^{3,1} \times S$ agree with the geometric description of F-theory on $X$. Reversing our observation, we also obtain a powerful means to determine the low-energy description of F-theory on more general $X$. Namely, if $X$ is given by an arbitrary unfolding of the singularity over $S$ as in (3.25), then F-theory on $X$ is still described at low-energies by the partially twisted Yang-Mills theory on $\mathbb{R}^{3,1} \times S$, but that Yang-Mills theory is now in a background for which $\varphi$ has a nontrivial, necessarily holomorphic expectation value, determined by the coefficients in the unfolding of the ADE-singularity.

\subsection{The Four-Dimensional Effective Theory}

In this Section we begin our analysis of four-dimensional supersymmetric vacua on $\mathbb{R}^{3,1}$ associated to the partially twisted seven-brane theory. To this end, we first explain the regime of F-theory compactifications for which we expect our analysis to remain valid. In many topological quantum field theories, the primary purpose of the Lagrangian is to enforce the BPS equations of motion when the theory is studied classically at weak-coupling. In the present case, however, the role of the eight-dimensional partially twisted Lagrangian is more central because it determines the Yukawa couplings in the low-energy effective theory on $\mathbb{R}^{3,1}$. Additional features of the partially twisted eight-dimensional theory are presented in Appendices B and C.

In this subsection we extract the massless spectrum and F-terms of the fourdimensional effective theory. Because this information only depends on holomorphic data and not the Kähler metric, general arguments suggest that we can reliably compute this result at large volume and then extrapolate to the regime of small volume, if desired. On the other hand, the D-terms of the four-dimensional effective theory will in general receive quantum corrections. In the regime of large volume, these effects can in principle be computed by integrating out the corresponding massive 
Kaluza-Klein modes which arise from compactification on $S$.

More precisely, the essential point which allows us to perform our analysis at large volume is that we may view the supersymmetry transformation $\bar{\delta}=\bar{\delta}_{\dot{1}}+\bar{\delta}_{\dot{2}}$ as a BRST-operator. Because the states in the cohomology ${ }^{12}$ of $\bar{\delta}$ transform in massless $\mathcal{N}=1$ chiral multiplets, for the purposes of analyzing these states and their superpotential interactions, we are free to take $\omega$ to be arbitrarily large. This observation is very important, since it underlies our classical analysis of the effective theory on $\mathbb{R}^{3,1}$ obtained by compactification of the eight-dimensional Yang-Mills theory on $S$. Indeed, this fact allows us to reliably compute both the massless spectrum and superpotential of the resulting theory.

From the perspective of the four-dimensional effective description in $\mathbb{R}^{3,1}$, the eight-dimensional fields of the partially twisted theory are packaged as two $\mathcal{N}=1$ chiral multiplets $\left(A_{\bar{m}}, \psi_{\alpha \bar{m}}\right)$ and $\left(\varphi_{m n}, \chi_{\alpha m n}\right)$, and a single vector multiplet $\left(A_{\mu}, \eta_{\alpha}\right)$. In rigid $\mathcal{N}=1$ superspace, the chiral multiplets then organize into chiral superfields $\boldsymbol{\Phi}_{m n}$ and $\mathbf{A}_{\bar{m}}$ with lowest bosonic components $\varphi_{m n}$ and $A_{\bar{m}}$, respectively. Letting $F_{S}$ denote the restriction of the eight-dimensional field strength $F$ to $S, F_{S}^{(0,2)}$ corresponds to the lowest bosonic component of an $\mathcal{N}=1$ chiral superfield $\mathbf{F}_{S}^{(0,2)}$ whose remaining components can be determined from the explicit off-shell transformations presented in Appendix C. Summarizing the end result of Appendix C, the partially twisted action in eight dimensions consists of contributions from $\bar{\delta}$ trivial terms and an F-term:

$$
I_{S}=\int_{\mathbb{R}^{3,1} \times S} d^{4} x d^{2} \bar{\theta} \mathcal{O}-\int_{\mathbb{R}^{3,1} \times S} d^{4} x d^{2} \theta \operatorname{Tr}\left(\mathbf{F}_{S}^{(0,2)} \wedge \boldsymbol{\Phi}\right),
$$

where $\mathcal{O}$ is a gauge invariant operator which is $\bar{\delta}$ trivial. Here and throughout, ' $\operatorname{Tr}$ ' generally denotes a negative-definite invariant quadratic form on the Lie algebra of the gauge group $G_{S}$. As standard, we normalize 'Tr' such that for $G_{S}=S U(n)$ this form corresponds to the trace in the fundamental representation. Also, $d^{4} x$ denotes the standard measure on $\mathbb{R}^{3,1}$.

The organization of the rest of this subsection is as follows. We first derive the BPS equations of motion for the fields of the partially twisted eight-dimensional theory. The massless spectrum of particles correspond to zero mode solutions on $S$ in

\footnotetext{
${ }^{12}$ Because we effectively consider cohomology with respect to the pair of supercharges $\bar{\delta}_{\dot{\alpha}}$, this topological field theory is of the "balanced" type discussed in [30].
} 
the presence of a non-trivial supersymmetric background gauge field configuration. These zero modes are represented by elements of an appropriate bundle valued Dolbeault cohomology group. After this analysis, we show how the four-dimensional superpotential descends from the partially twisted eight-dimensional theory. When $S$ is a Hirzebruch or del Pezzo surface, a general vanishing theorem for cohomology groups forbids any classical Yukawa couplings. In subsequent sections we resolve this issue by treating a more general class of F-theory compactifications.

\subsubsection{BPS Equations}

To begin our analysis of four-dimensional supersymmetric vacua, we first present the BPS equations of motion for the eight-dimensional fields of the partially twisted theory. In a supersymmetric vacuum, the variations of all of the eight-dimensional fermions must vanish. These conditions determine the BPS equations of motion. From the viewpoint of the four-dimensional effective theory, these equations follow from the requirement that in a supersymmetric vacuum the corresponding effective potential is both D- and F-flat. In this subsection we present these conditions in the regime of large volume for $S$. Whereas the D-flatness conditions will in general receive perturbative corrections away from the regime of large volume, up to nonperturbative effects, the equations of motion derived from the F-terms will remain unchanged.

The on-shell supersymmetry transformations of the eight-dimensional fields of the partially twisted theory may be found in Appendix B. From the variations of $\eta_{\beta}$ and $\bar{\eta}_{\dot{\beta}}$, we see that both the self-dual and anti-self-dual components of the curvature $F_{\mu \nu}$ on $\mathbb{R}^{3,1}$ must vanish, so that

$$
F_{\mu \nu}=0 .
$$

From the variations of $\psi_{\beta \bar{m}}, \chi_{\beta m n}$ and their conjugates, we also see that the mixed components of the curvature along $\mathbb{R}^{3,1} \times S$ vanish,

$$
F_{\mu m}=F_{\mu \bar{m}}=0 .
$$

Similarly, both $\varphi$ and $\bar{\varphi}$ satisfy

$$
D_{\mu} \varphi=D_{\mu} \bar{\varphi}=0 .
$$


The more interesting BPS equations involve the behavior of the gauge field and the twisted scalars $(\varphi, \bar{\varphi})$ on $S$. First, from the variations of the fermions $\chi_{\alpha}$ and $\bar{\chi}_{\dot{\alpha}}$ in (B.2), we see that the components of the curvature on $S$ of holomorphic/antiholomorphic type $(2,0)$ and $(0,2)$ both vanish,

$$
F_{S}^{(0,2)}=F_{S}^{(2,0)}=0
$$

Hence the $(0,1)$ component $\bar{\partial}_{A}$ of the covariant derivative associated to the gauge field on $S$ endows the $G_{S}$-bundle $P$ with a holomorphic structure, since $\bar{\partial}_{A}^{2}=F_{S}^{(0,2)}=0$. The variation of the fermions in (B.2) also implies a holomorphy condition on $\varphi$ as well as an anti-holomorphy condition on $\bar{\varphi}$,

$$
\bar{\partial}_{A} \varphi=\partial_{A} \bar{\varphi}=0
$$

Finally, from the variations of $\eta_{\alpha}$ and $\bar{\eta}_{\dot{\alpha}}$, the $(1,1)$-component of the curvature on $S$ must be related to the commutator of $\varphi$ and $\bar{\varphi}$ as:

$$
\omega \wedge F_{S}+\frac{i}{2}[\varphi, \bar{\varphi}]=0
$$

where $\omega$ is the Kähler form on $S$.

Now, as the discussion in Appendix C shows, the holomorphy conditions in (3.32) and (3.33) are both associated to the vanishing of F-terms in the language of $\mathcal{N}=1$ supersymmetry. Neither of these F-term conditions involves the Kähler metric on $S$, and we do not expect them to receive corrections, at least in perturbation theory. On the other hand, the supersymmetry condition in (3.34) involves the vanishing of a D-term, and this condition manifestly depends upon the Kähler metric on $S$. In general, the D-term condition in (3.34) can therefore receive perturbative corrections as we integrate out massive Kaluza-Klein modes on $S$. However, if the volume of $S$ is sufficiently large, we still expect solutions to the classical D-term equation in (3.34) to determine supersymmetric configurations for $A, \varphi$, and $\bar{\varphi}$ on $S$.

In much of this paper we shall primarily consider vacua such that $\varphi=0$. In this case, equations (3.32) and (3.34) correspond to the usual Hermitian Yang-Mills equations, which are satisfied when the connection on $P$ is anti-self-dual and the gauge field describes an instanton on $S$.

We now discuss one immediate consequence of (3.34) which is especially helpful 
for explicit computations with line bundles. Given a holomorphic gauge bundle $\mathcal{T}$ transforming in a unitary representation $T$ of some subgroup of $G_{S}$ which we denote by $H_{S}$, we note that the first Chern class of $\mathcal{T}$ admits a Chern-Weil representation as $c_{1}(\mathcal{T})=\frac{i}{2 \pi} \operatorname{Tr}_{T}\left(F_{S}\right)$. When $\varphi=0$, tracing over equation (3.34) in the representation $T$ yields the condition:

$$
\omega \wedge c_{1}(\mathcal{T})=0 \in H^{2}(S ; \mathbb{R})
$$

where $\omega$ denotes the Kähler form on $S$. In the special case where $\mathcal{T}$ is a line bundle and $\varphi=0$, equation (3.34) is equivalent to equation (3.35). Because $c_{1}(\mathcal{T})$ is also quantized as an element in the integral cohomology of $S$, equation (3.35) can only be solved for generic $\omega$ if $c_{1}(\mathcal{T})=0$. Conversely, if (3.35) is to admit a solution with $c_{1}(\mathcal{T}) \neq 0$, we must assume that $\omega$ is non-generic. Said differently, a supersymmetric gauge field configuration stabilizes a Kähler modulus in the compactification. Some examples of supersymmetric line bundles for $S$ a Hirzebruch or del Pezzo surface are presented in Appendix E.

Before closing this subsection, we note that the BPS equations in (3.32), (3.33), and (3.34) have certainly been considered before. These equations on $S$ are precisely $^{13}$ the equations which arise from the topological twist of four-dimensional, $\mathcal{N}=4$ supersymmetric Yang-Mills theory studied in [24] when that theory is specialized to a Kähler four-manifold. Indeed, the partial twisting of the eight-dimensional theory we are considering is identical to the theory of [24] in the Kähler case.

\subsubsection{Bulk Spectrum of Massless Particles}

In direct analogy with heterotic Calabi-Yau compactifications, the massless spectrum of the seven-brane theory wrapping $S$ originates from a potentially non-trivial background gauge field configuration on $S$ with values in some subgroup $H_{S}$. More precisely, the spectrum in four dimensions is determined by bundle valued Dolbeault cohomology groups $H \frac{i}{\partial}$ on $S$ where $i=0,1,2$. This fact allows us to express the total massless spectrum in a given representation of the unbroken gauge group in terms of a topological index formula. The end result of this analysis is that the number of

\footnotetext{
${ }^{13}$ Due to the fact that we follow slightly different conventions from [24], a factor of ' $i / 2$ ' appears in (3.34) which is otherwise absent in [24].
} 
generations minus anti-generations in a representation $\tau$ is:

$$
n_{\tau}-n_{\tau^{*}}=-\int_{S} c_{1}(\mathcal{T}) c_{1}(S)
$$

where $\tau^{*}$ denotes the dual representation to $\tau$. Letting $\Gamma_{S}$ denote the maximal subgroup of $G_{S}$ such that $G_{S} \supset \Gamma_{S} \times H_{S}$, in the above, $\mathcal{T}$ denotes a bundle transforming in a representation $T$ of $H_{S}$ such that the decomposition of the adjoint representation of $G_{S}$ to $\Gamma_{S} \times H_{S}$ contains the representation $(\tau, T)$.

We now describe in further detail the massless spectrum. Our starting point is a supersymmetric background configuration for the gauge field and the twisted scalars $(\varphi, \bar{\varphi})$ on $S$. For our applications, we shall primarily be interested in configurations with $\varphi=0$ or more generally configurations such that $[\varphi, \bar{\varphi}]=0$. Under these assumptions, the BPS equations state that the principal $G_{S^{-}}$bundle $P$ carries an anti-self-dual connection, corresponding to an instanton on $S$. While the case of non-vanishing commutator for the internal four dimensional $\mathcal{N}=4$ twisted theory on $S$ has been studied in [24], it would be interesting to interpret such vacua from the perspective of the seven-brane theory. ${ }^{14}$

The representation content of the particle spectrum is fixed by decomposing the adjoint representation of $G_{S}$ into irreducible representations of $\Gamma_{S} \times H_{S}$ :

$$
\operatorname{ad}\left(G_{S}\right) \cong \bigoplus_{j} \tau_{j} \otimes T_{j}
$$

A similar decomposition holds for the bundle $\operatorname{ad}(P)$. In the obvious notation, we let $\mathcal{T}_{j}$ denote the corresponding bundle which transforms as a representation $T_{j}$ of $H_{S}$.

Given an instanton on $S$ with structure group $H_{S}$, the unbroken low energy gauge group is given by the commutant subgroup of $H_{S}$ in $G_{S}$. We note that in some cases such as when $H_{S}$ contains semi-simple $U(1)$ factors, $\Gamma_{S}$ corresponds to a proper subgroup of the four-dimensional gauge group. For example, when $G_{S}=E_{6}$ and $H_{S}=U(1)$, the commutant is $G_{4 d}=S O(10) \times U(1)$. On the other hand, in many cases this $U(1)$ factor is anomalous and therefore decouples from the low energy dynamics via the Green-Schwarz mechanism.

\footnotetext{
${ }^{14}$ When this commutator does not vanish, it is tempting to speculate that the seven-brane experiences an analogue of the Myers effect. We thank M. Wijnholt for suggesting this possibility.
} 
The massless spectrum on $\mathbb{R}^{3,1}$ is given by zero mode solutions on $S$ in the presence of a potentially non-trivial background gauge field configuration. Because the partial twisting on $S$ automatically produces a covariantly constant supercurrent along $S$, each fermionic zero mode possesses a bosonic counterpart. It therefore suffices to specify the spectrum of massless fermions in the four-dimensional effective theory. By inspection of the topological action, in a background with $\varphi=\bar{\varphi}=0$, the equations of motion for the zero modes state that the fermions $\bar{\eta}_{\dot{\alpha}}, \psi_{\alpha}$, and $\bar{\chi}_{\dot{\alpha}}$ in (3.18) are annihilated by the operators $\bar{\partial}_{A}$ and $\bar{\partial}_{A}^{\dagger}$ and hence by the Laplacian $\triangle_{\bar{\partial}}=\bar{\partial}_{A}^{\dagger} \bar{\partial}_{A}+\bar{\partial}_{A} \bar{\partial}_{A}^{\dagger}$.

Via the standard Hodge isomorphism, we then obtain massless fermions on $\mathbb{R}^{3,1}$ from the following Dolbeault cohomology groups on $S$,

$$
H \frac{0}{\partial}(S, \operatorname{ad}(P)) \oplus H \frac{1}{\partial}(S, \operatorname{ad}(P)) \oplus H_{\frac{2}{\partial}}(S, \operatorname{ad}(P))
$$

where the massless modes for $\bar{\eta}_{\dot{\alpha}}, \psi_{\alpha}$ and $\bar{\chi}_{\dot{\alpha}}$ are associated to elements of $H_{\frac{0}{\partial}}^{0}, H_{\bar{\partial}} \frac{1}{}$ and $H_{\frac{2}{\partial}}$, respectively. Note that the natural even/odd grading on the cohomology correlates with the the chirality of the corresponding massless fermions on $\mathbb{R}^{3,1}$. Labelling the representation content of a four-dimensional massless chiral field by an additional subscript, the decomposition of $\operatorname{ad}(P)$ in the analogue of equation (3.37) implies that the zero modes in a representation $\tau_{j}$ of $\Gamma_{S}$ belong to the $\mathcal{T}_{j}$ bundle valued cohomology groups:

$$
\begin{aligned}
\bar{\eta}_{\dot{\alpha} \tau_{j}} & \in H \frac{0}{\partial}\left(S, \mathcal{T}_{j}\right) \\
\psi_{\alpha \tau_{j}} & \in H \frac{1}{\partial}\left(S, \mathcal{T}_{j}\right) \\
\bar{\chi}_{\dot{\alpha} \tau_{j}} & \in H \frac{2}{\partial}\left(S, \mathcal{T}_{j}\right)
\end{aligned}
$$

Further, when $\tau_{j}$ is a complex representation, the CPT conjugates of $\bar{\eta}_{\dot{\alpha} \tau_{j}}$ and $\bar{\chi}_{\dot{\alpha} \tau_{j}}$ correspond to chiral spinors in the complex conjugate representation $\tau_{j}^{*}$.

The same remarks hold for the fermions $\eta_{\alpha}, \bar{\psi}_{\dot{\alpha}}$, and $\chi_{\alpha}$ in (3.19) which are related to the zero modes described above by CPT conjugation. Because the zeromode wavefunctions for $\eta_{\alpha}, \bar{\psi}_{\dot{\alpha}}$, and $\chi_{\alpha}$ are naturally anti-holomorphic on $S$, we identify those wavefunctions with elements in the conjugate to (3.38):

$$
\overline{H_{\bar{\partial}}^{0}(S, \operatorname{ad}(P))} \oplus \overline{H_{\frac{1}{\partial}}(S, \operatorname{ad}(P))} \oplus \overline{H_{\frac{2}{\partial}}^{2}(S, \operatorname{ad}(P))} .
$$


Because algebraic geometry typically deals with holomorphic objects, this is slightly awkward. A holomorphic description for the zero modes described by (3.42) is obtained using the isomorphism of vector spaces ${ }^{15}$ :

$$
\overline{H_{\bar{\partial}}^{p}(S, \operatorname{ad}(P))} \cong H_{\bar{\partial}}^{p}(S, \operatorname{ad}(P))^{*}
$$

Physically, dualization corresponds to CPT conjugation. It is now immediate that the chiral and anti-chiral spectrum for fields in a representation $\tau_{j}$ of $\Gamma_{S}$ is given by the cohomology groups:

$$
\begin{aligned}
\text { chiral: } & H_{\frac{0}{\partial}}\left(S, \mathcal{T}_{j}^{*}\right)^{*} \oplus H \frac{1}{\partial}\left(S, \mathcal{T}_{j}\right) \oplus H_{\frac{2}{\partial}}\left(S, \mathcal{T}_{j}^{*}\right)^{*} \\
\text { anti-chiral: } & H_{\frac{0}{\partial}}\left(S, \mathcal{T}_{j}\right) \oplus H \frac{1}{\partial}\left(S, \mathcal{T}_{j}^{*}\right)^{*} \oplus H_{\frac{2}{\partial}}\left(S, \mathcal{T}_{j}\right) .
\end{aligned}
$$

Note in particular that the resulting spectrum is automatically CPT-invariant.

In principle, the spectrum is now determined entirely in terms of the Dolbeault cohomology groups of (3.44) for the bundle $\mathcal{T}_{j}$ associated with a representation $\tau_{j}$ which appear in the decomposition of $\operatorname{ad}(P)$. In general, these cohomology groups are not topological objects because they depend holomorphically upon the complex structure moduli for $S$ and the vector bundles $\mathcal{T}_{j}$ and $\mathcal{T}_{j}^{*}$. Nevertheless, returning to (3.44), the analogous expression for $\tau_{j}^{*}$ interchanges all instances of $\mathcal{T}_{j}$ and $\mathcal{T}_{j}^{*}$. The number of massless four-dimensional chiral fields in the representation $\tau_{j}$ minus the number in $\tau_{j}^{*}$ is therefore:

$$
\begin{aligned}
n_{\tau_{j}}-n_{\tau_{j}^{*}} & =h^{0}\left(S, \mathcal{T}_{j}^{*}\right)+h^{1}\left(S, \mathcal{T}_{j}\right)+h^{2}\left(S, \mathcal{T}_{j}^{*}\right) \\
& -\left(h^{0}\left(S, \mathcal{T}_{j}\right)+h^{1}\left(S, \mathcal{T}_{j}^{*}\right)+h^{2}\left(S, \mathcal{T}_{j}\right)\right) \\
& =\chi\left(S, \mathcal{T}_{j}^{*}\right)-\chi\left(S, \mathcal{T}_{j}\right)
\end{aligned}
$$

where as usual, $h^{i}=\operatorname{dim}_{\mathbb{C}} H^{i}$ and the Euler character $\chi=h^{0}-h^{1}+h^{2}$ is a topological invariant.

The explicit values of the above Euler characters are determined by an index

\footnotetext{
${ }^{15}$ This isomorphism is obtained as follows. We first observe that the Kähler metric on $S$ and the invariant form ' $T r$ ' on the Lie algebra of $G_{S}$ together define the obvious inner product between elements in (3.38) and (3.42). The $p$-form indices are contracted along $S$ using the Kähler metric with all Lie algebra indices contracted using the form ' $\mathrm{Tr}$ '.
} 
formula:

$$
\begin{aligned}
\chi(S, \mathcal{T}) & =\int_{S} \operatorname{ch}(\mathcal{T}) \operatorname{Td}(S) \\
& =\int_{S}\left(\frac{\operatorname{rk}(\mathcal{T})}{12}\left[c_{1}(S)^{2}+c_{2}(S)\right]+\frac{1}{2} c_{1}(\mathcal{T}) c_{1}(S)+\frac{1}{2}\left[c_{1}(\mathcal{T})^{2}-2 c_{2}(\mathcal{T})\right]\right) .
\end{aligned}
$$

Because $c_{1}(\mathcal{T})=-c_{1}\left(\mathcal{T}^{*}\right)$ and $c_{2}(\mathcal{T})=c_{2}\left(\mathcal{T}^{*}\right)$, the number of chiral generations minus anti-generations in a representation $\tau_{j}$ is:

$$
n_{\tau_{j}}-n_{\tau_{j}^{*}}=-\int_{S} c_{1}\left(\mathcal{T}_{j}\right) c_{1}(S) .
$$

In the rigid case where $S$ is either a Hirzebruch of del Pezzo surface, the number of generations and anti-generations are each computed by a distinct index. This is a consequence of the general vanishing theorem established in Appendix E which shows that for an arbitrary supersymmetric gauge field configuration, $H_{\bar{\partial}}\left(S, \mathcal{T}_{j}\right)=0 .{ }^{16}$ Moreover, when the holomorphic bundle $\mathcal{T}_{j}$ is irreducible and non-trivial (meaning $\left.\mathcal{T}_{j} \neq \mathcal{O}_{S}\right)$, the discussion in Appendix E also shows that $H_{\bar{\partial}}\left(S, \mathcal{T}_{j}\right)=0$. Combining these facts yields:

$$
\begin{gathered}
n_{\tau_{j}}=h^{1}\left(S, \mathcal{T}_{j}\right)=-\chi\left(S, \mathcal{T}_{j}\right) \\
n_{\tau_{j}^{*}}=h^{1}\left(S, \mathcal{T}_{j}^{*}\right)=-\chi\left(S, \mathcal{T}_{j}^{*}\right) .
\end{gathered}
$$

\subsubsection{Bulk Yukawa Couplings}

In this section we summarize the Yukawa couplings of the four-dimensional effective theory for a seven-brane wrapping a general complex surface $S$. We also find that when $S$ is either a Hirzebruch or del Pezzo surface, all of the classical Yukawa couplings vanish.

\footnotetext{
${ }^{16} \mathrm{~A}$ related vanishing theorem is as follows. Note that because $\varphi$ transforms as a section of $K_{S} \otimes \operatorname{ad}(P)$, a zero mode solution for $\varphi$ would imply that $H \frac{i}{\partial}\left(S, K_{S}^{n}\right)$ is non-zero for some $n>0$. Because $K_{S}$ is a strictly negative line bundle, the Kodaira vanishing theorem establishes the absence of such solutions.
} 
When $S$ is a general complex surface, the four-dimensional Yukawa couplings for the zero mode solutions catalogued by (3.44) originate from the last term in the action of equation (3.28). Labelling the zero mode solutions by $\alpha, \beta$ and $\gamma$, the Yukawa couplings of the four-dimensional effective are therefore determined by the superpotential:

$$
d_{\alpha \beta \gamma}=-\int_{S} h_{i j k} \mathbf{A}^{\beta, i} \wedge \mathbf{A}^{\gamma, j} \wedge \boldsymbol{\Phi}^{\alpha, k},
$$

where $i, j, k$ and $h_{i j k}$ respectively denote group theory indices and the structure constants of $G_{S}$ associated with breaking $G_{S}$ to the subgroup $\Gamma_{S} \times H_{S}$.

Using the isomorphism of equation (3.43), a non-trivial Yukawa coupling between three representations $\tau_{1}, \tau_{2}, \tau_{3}$ of $\Gamma_{S}$ corresponds to a tri-linear map:

$$
H \frac{1}{\partial}\left(S, \mathcal{T}_{1}\right) \otimes H \frac{1}{\partial}\left(S, \mathcal{T}_{2}\right) \otimes H_{\frac{\partial}{\partial}}\left(S, \mathcal{T}_{3}^{*}\right)^{*} \rightarrow \mathbb{C}
$$

Note in particular that when $S$ is either a Hirzebruch or del Pezzo surface, the vanishing theorem used in the previous section to constrain the zero mode content also requires all Yukawa couplings to vanish. This is unacceptable from the viewpoint of phenomenology. In subsequent sections we shall rectify this deficiency by treating a more general class of F-theory compactifications which couple the partially twisted theory to a six-dimensional defect theory with matter localized along Riemann surfaces in $S$.

\subsection{Bulk Toy Models}

Using the above results, we now present some toy models which illustrate how both non-chiral and chiral matter can originate from a supersymmetric gauge field configuration on $S$. Initially, we take $S$ to be a general del Pezzo surface. We first show that instanton solutions on $S$ with structure group $S U(n)$ can generate a non-trivial massless spectrum in four dimensions. Nevertheless, we find that in such cases the resulting spectrum is always non-chiral because the corresponding instanton solutions have vanishing first Chern class. We next present an $S O(10)$ model with three chiral generations transforming in the $\mathbf{1 6}$ spinor representation.

To generate zero modes on $S$ which can descend to massless fields in four dimensions, we introduce an instanton solution with structure group $S U(n) \subset G_{S}$ on $S$ 
with instanton number $k$. As an example, suppose that the decomposition of the adjoint of $G_{S}$ to $\Gamma_{S} \times S U(n)$ contains the representation $(\tau, n)$. Assuming that a non-trivial instanton solution on $S$ exists, the resulting number of massless chiral four-dimensional fields in the representation $\tau$ is:

$$
n_{\tau}=k-n
$$

The existence of zero mode solutions thus imposes the condition $k \geq n$.

While the explicit parameterization of such an instanton solution is quite nontrivial, it is enough to check that the moduli space $\mathcal{M}_{k}$ of solutions with instanton number $k$ is non-trivial. The dimension of $\mathcal{M}_{k}$ is:

$$
\operatorname{dim} \mathcal{M}_{k}=4 k n-\left(n^{2}-1\right) \frac{(\chi+\sigma)}{2}
$$

where $\chi$ and $\sigma$ respectively denote the Euler character and signature of $S$. When $S$ is a del Pezzo surface, this becomes:

$$
\operatorname{dim} \mathcal{M}_{k}=4 k n-2\left(n^{2}-1\right)
$$

The condition $n_{\tau} \geq 0$ implies:

$$
\operatorname{dim} \mathcal{M}_{k}=4 k n-2\left(n^{2}-1\right) \geq 2 n^{2}+2>0
$$

so that there exists a moduli space of solutions in this case which can generate massless chiral four-dimensional fields in the representation $\tau$ of $\Gamma_{S}$. A similar analysis shows that when $\tau$ is a complex representation, the number of massless chiral fourdimensional fields in the complex conjugate representation $\tau^{*}$ precisely matches the number transforming in $\tau$, so that the resulting spectrum is non-chiral.

In fact, we now show that for arbitrary $S$, an $S U(n)$ instanton solution cannot generate a chiral spectrum in four dimensions. Indeed, because the adjoint of $G_{S}$ is a real representation, the decomposition to $\Gamma_{S} \times S U(n)$ will contain the representations $\left(\tau_{j}, T_{j}\right)$ and $\left(\tau_{j}^{*}, T_{j}^{*}\right)$ when the representation $\tau_{j}$ is complex. Next recall from equation (3.36) that the net number of generations in $\tau_{j}$ minus the number in $\tau_{j}^{*}$ is $-c_{1}(S)$. $c_{1}\left(\mathcal{T}_{j}\right)$. Because all unitary representations of $S U(n)$ are traceless, $c_{1}\left(\mathcal{T}_{j}\right)$ vanishes.

We now present an explicit toy model with chiral matter spectrum induced by a 
supersymmetric gauge field configuration such that tensor powers of the gauge bundle have non-zero first Chern class. To this end, we take a seven-brane wrapping the Hirzebruch surface $S=\mathbb{F}_{1}$ with bulk gauge group $G_{S}=E_{6}$. By turning on an appropriate supersymmetric $U(1)$ gauge field configuration defined by a line bundle $\mathcal{L}$ on $S, E_{6}$ will break to $S O(10) \times U(1)$. To determine the chiral spectrum of this theory, we note that the adjoint of $E_{6}$ decomposes as:

$$
\begin{aligned}
& E_{6} \supset S O(10) \times U(1) \\
& \mathbf{7 8} \rightarrow \mathbf{4 5}_{0}+\mathbf{1}_{0}+\mathbf{1 6}_{-3}+\overline{\mathbf{1 6}}_{+3}
\end{aligned}
$$

where we have chosen an integral normalization of the $U(1)$ charge. Invoking the vanishing theorem proved in Appendix E, the zero modes of the Dolbeault operator for each representation are classified by:

$$
\begin{aligned}
& \mathbf{1 6}_{-3} \in H \frac{1}{\partial}\left(S, \mathcal{L}^{-3}\right) \\
& \overline{\mathbf{1 6}}_{+3} \in H \frac{1}{\partial}\left(S, \mathcal{L}^{+3}\right) .
\end{aligned}
$$

All supersymmetric line bundles of the Hirzebruch surfaces are classified in Appendix E. The result is that a line bundle is supersymmetric for some Kähler class if and only if there exist integers $a$ and $b$ such that $a b<0$ so that $\mathcal{L}$ is given by:

$$
\mathcal{L}=\mathcal{O}_{\mathbb{F}_{1}}(a f+b \sigma),
$$

with $f$ and $\sigma$ as in Appendix A. Using the fact that $c_{1}\left(\mathbb{F}_{1}\right)=3 f+2 \sigma$, the number of four-dimensional massless fields in the $\mathbf{1 6}$ now follows from equations (3.50) and (3.52):

$$
\begin{aligned}
n_{\mathbf{1 6}} & =-\left(\operatorname{rk}\left(\mathcal{L}^{-3}\right)+\frac{1}{2} c_{1}\left(\mathcal{L}^{-3}\right) \cdot(3 f+2 \sigma)+\frac{1}{2} c_{1}\left(\mathcal{L}^{-3}\right) \cdot c_{1}\left(\mathcal{L}^{-3}\right)\right) \\
& =-\left(1-\frac{3}{2}(2 a+b)+\frac{9}{2}\left(2 a b-b^{2}\right)\right)
\end{aligned}
$$

where in the above we have used the fact that the Todd genus of $S$ is unity for a 
Hirzebruch surface. A similar computation for the number of $\overline{\mathbf{1 6}}$ 's yields:

$$
n_{\overline{\mathbf{1 6}}}=-\left(1+\frac{3}{2}(2 a+b)+\frac{9}{2}\left(2 a b-b^{2}\right)\right) .
$$

It is amusing that the net number of $\mathbf{1 6}$ 's is always a multiple of three:

$$
n_{16}-n_{\overline{16}}=3(2 a+b)
$$

so that when $a=1$ and $b=-1$, we have precisely three generations in the $\mathbf{1 6}$ of $S O(10)$.

Let us now explicitly check that the zero mode content contains no contribution from $H_{\frac{0}{\partial}}^{0}$ and $H_{\bar{\partial}}$. Because $f$ and $\sigma$ generate the cone of all effective classes in $H_{2}(S, \mathbb{Z})$, when $a$ and $b$ have opposite signs, any purported global section of $\mathcal{L}$ will have a pole along either $\sigma$ or $f$. This implies $H_{\frac{0}{\partial}}\left(S, \mathcal{L}^{n}\right)$ is trivial for all $n$ a non-trivial integer. Moreover, by Serre duality we have:

$$
H \frac{2}{\partial}\left(S, \mathcal{O}_{\mathbb{F}_{1}}(n a f+n b \sigma) \simeq H \frac{0}{\partial}\left(S, \mathcal{O}_{\mathbb{F}_{1}}(-(n a+3) f-(n b+2) \sigma)^{*}\right.\right.
$$

In order for $H_{\frac{2}{\partial}}\left(S, \mathcal{L}^{n}\right)$ to be non-trivial, both $n a$ and $n b$ must be negative. Because this is not possible, it follows that the massless spectrum is completely characterized by $H \frac{1}{\partial}$.

\section{Intersecting Seven-Branes in F-Theory}

So far, we have analyzed the low energy effective description for F-theory on a class of very special elliptic Calabi-Yau fourfolds $X$ given by canonical $A D E$ singularities over a smooth, compact Kähler surface $S$. We began by considering such models not because they are particularly realistic, but because they are particularly simple: F-theory on the local Calabi-Yau fourfold $X$ is described at low energies by the partially twisted Yang-Mills theory which lives on the worldvolume of the sevenbrane wrapping $\mathbb{R}^{3,1} \times S$.

In this Section, our goal is to study the low-energy effective description for $F$ theory on a much more general class of local elliptic Calabi-Yau fourfolds. From a physical perspective, these new F-theory backgrounds will contain intersecting seven- 
branes of various $A D E$-types. From a mathematical perspective, we will allow the singular locus $\Delta \subset B$ of the elliptic fibration of $X$ to be reducible, so that $\Delta$ consists of several components of colliding singularities in the threefold $B$.

For the sake of concreteness, we start in Section 4.1 by presenting simple local models for elliptic Calabi-Yau fourfolds associated to intersecting seven-branes in Ftheory. Of course, as noted long ago [22] in the context of F-theory on elliptic CalabiYau threefolds, when two seven-branes intersect along a common six-dimensional subspace, one finds additional light degrees of freedom localized at the intersection and charged under the worldvolume gauge group carried by each seven-brane, analogous to the bifundamental matter that arises at the intersection of ordinary D-branes. Somewhat surprisingly, the effective description of this light charged matter seems never to have been worked out for $\mathcal{N}=1$ supersymmetric F-theory models.

To remedy that gap, we consider in Section 4.2 the case that our original sevenbrane wrapping the surface $S$ intersects another seven-brane wrapping a surface $S^{\prime}$ transversely along a smooth complex curve $\Sigma=S \cap S^{\prime}$. As we explain, the effective dynamics of the light, charged degrees of freedom living on the intersection $\mathbb{R}^{3,1} \times \Sigma$ are captured by a partially twisted six-dimensional defect theory which is jointly coupled to the bulk, eight-dimensional Yang-Mills theories living on the two sevenbranes. If $S^{\prime}$ is non-compact, as will often be the case in our local models, then the fields in the twisted Yang-Mills theory on $\mathbb{R}^{3,1} \times S^{\prime}$ are non-dynamical background fields, and we simply consider a twisted defect theory on $\mathbb{R}^{3,1} \times \Sigma$ coupled to the twisted Yang-Mills theory on $\mathbb{R}^{3,1} \times S$ introduced in Section 3 .

We next present evidence that this more involved theory accurately describes the corresponding compactification in F-theory. To this end, in Section 4.3 we match the additional moduli of the defect theory on $\Sigma$ to the moduli of F-theory on $X$. This match is quite interesting, since it relies upon the interpretation of the defect theory on $\Sigma$ as inducing a surface operator for the bulk gauge theory on $S$.

Having argued that the partially twisted theory does indeed describe a more genral class of F-theory compactifications, in Section 4.4 we study the resulting spectrum of massless charged matter and effective superpotential in four dimensions. Once again, the spectrum of massless charged matter is determined by certain Dolbeault cohomology groups on $\Sigma$, and the net chirality in four dimensions is given by a corresponding index. We also find generically non-zero Yukawa couplings involving two chiral superfields derived from $\Sigma$ and one from the bulk theory on $S$. 


\subsection{Colliding Singularities and Intersecting Seven-Branes}

In this Section we present geometries for a more general class of F-theory vacua which contain intersecting seven-branes. To describe these intersections geometrically in terms of colliding singularities, we begin by generalizing the local model for the elliptically-fibered Calabi-Yau fourfold $X$ given in Section 3.1.

Once again, $X$ will be the total space of an elliptic $K 3$-fibration over the compact surface $S$,

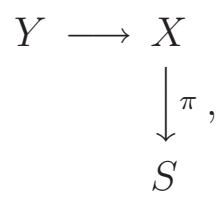

where $Y$ remains a local, elliptically-fibered $K 3$ given as a hypersurface in $\mathbb{C}^{3}$. However, we now allow the singularities in $Y$ to vary from point to point over $S$. At points where the generic singularity on $S$ enhances, two seven-branes intersect.

Intersecting Seven-Branes of A-Type

As a first example, we exhibit a local model for $X$ that describes a compact 7brane of type $A_{n}$ which wraps $S$ and intersects another, non-compact seven-brane of type $A_{m}$ along an effective divisor $\Sigma \subset S$. The fibering of $Y$ over $S$ is necessarily more complicated in this situation, so we generalize (3.5) by allowing the affine coordinates $(x, y, z)$ on $\mathbb{C}^{3}$ to transform over $S$ as sections of the rank three bundle:

$$
V=L \oplus L \oplus K_{S}
$$

At the moment, $L$ is an arbitrary line bundle over $S$, and the motivation behind this particular ansatz for $V$ will be clear momentarily.

We now take $Y$ to be the hypersurface in $V$ given by

$$
y^{2}=x^{2}+\alpha^{m+1} z^{n+1}, \quad \alpha \in H \frac{0}{\partial}\left(S, \mathcal{O}_{S}(\Sigma)\right)
$$

Here $\alpha$ is a holomorphic section of the line bundle $\mathcal{O}_{S}(\Sigma)$ associated to the divisor $\Sigma$, along which we assume $\alpha$ vanishes to first-order. The equation (4.3) is homogeneous if we take $L^{2}=\mathcal{O}_{S}((m+1) \Sigma) \otimes K_{S}^{(n+1)}$. Note that the holomorphic two-form $(1 / y) d x \wedge d z$ on $Y$ automatically transforms as a section of $K_{S}$, so that $X$ is Calabi- 
Yau. $^{17}$

Away from $\Sigma, \alpha$ is non-vanishing, so (4.3) describes a generic $A_{n}$ singularity along $S$, identified as the zero-section in $V$. Therefore we have a seven-brane of type $A_{n}$ wrapping $S$. On the other hand, away from $S$ itself, the normal coordinate $z$ to $S \subset B$ is non-vanishing. Because $\alpha$ vanishes to first-order along $\Sigma$, we still find a generic $A_{m}$ singularity in the fiber of $K_{S}$ over $\Sigma$. Thus we also have a seven-brane of type $A_{m}$ extending in the fiber over $\Sigma$.

Over $\Sigma \subset S$, the $A_{m}$ and $A_{n}$ singularities collide and enhance to an $A_{m+n+1}$ singularity. That is, we have a transversal intesection of one seven-brane carrying the worldvolume gauge group $S U(n+1)$ with another seven-brane carrying the worldvolume gauge group $S U(m+1)$. As usual, we expect to find bifundamental matter localized along $\Sigma$ and transforming under $S U(n+1) \times S U(m+1)$ as

$$
(\mathbf{n}+\mathbf{1}, \overline{\mathbf{m}+\mathbf{1}}) \oplus(\overline{\mathbf{n}+\mathbf{1}}, \mathbf{m}+\mathbf{1}) .
$$

The local model for $X$ determined by (4.3) is only the simplest in a wide class of examples. For instance, given holomorphic sections

$$
\begin{aligned}
& t_{k} \in H \frac{0}{\partial}\left(S, K_{S}\right), \quad k=1, \ldots, n+1, \quad \sum_{k=1}^{n+1} t_{k}=0, \\
& u_{\ell} \in H \frac{0}{\partial}\left(S, \mathcal{O}_{S}(\Sigma)\right), \quad \ell=1, \ldots, m+1, \quad \sum_{\ell=1}^{m+1} u_{\ell}=0 \text {, }
\end{aligned}
$$

we can deform (4.3) to

$$
y^{2}=x^{2}+\prod_{\ell=1}^{m+1}\left(\alpha+u_{\ell}\right) \prod_{k=1}^{n+1}\left(z+t_{k}\right) .
$$

The 'unfolding' of the respective $A_{n}$ and $A_{m}$ singularities in equation (4.6) can be interpreted physically as a separation of the seven-branes in the original stacks wrapping $S$ and the fiber over $\Sigma$. Indeed, equation (4.6) describes seven-branes which

\footnotetext{
${ }^{17} \mathrm{As}$ in (3.8), the potentially half-integral nature of $L$ is an artifact associated to our particular parametrization for the $A_{n}$ singularity. If we instead take $x y=\alpha^{m+1} z^{n+1}$, no square-roots are necessary.
} 
now wrap the divisors in the threefold $B$ given by $z+t_{k}=0$ and $\alpha+u_{\ell}=0$.

Of course, if the canonical bundle $K_{S}$ has no holomorphic sections, or if $\alpha$ is the only holomorphic section of $\mathcal{O}_{S}(\Sigma)$ up to scale, then the local model for $X$ in (4.3) cannot be deformed as in (4.6). However, we can still generalize (4.6) as follows.

To simplify the notation, let us focus on the case $m=0$, for which we expand (4.6) as

$$
\begin{aligned}
y^{2} & =x^{2}+\alpha \prod_{k=1}^{n+1}\left(z+t_{k}\right) \\
& =x^{2}+\alpha\left[z^{n+1}+s_{2} z^{n-1}+\cdots+s_{n+1}\right] .
\end{aligned}
$$

where $s_{k}$ denotes the elementary symmetric polynomial of degree $k$ in the $n+1$ variables $t_{1}, \cdots, t_{n+1}$. If $m=0$ as in (4.7), then the hypersurface $Y$ is actually nonsingular along the locus where $\alpha$ vanishes, but the corresponding elliptic fibration has a singular fiber of Kodaira type $I_{1}$. A singularity of type $I_{1}$ is the generic singularity in an elliptic fibration, and corresponds to a seven-brane with worldvolume gauge group $U(1)$.

Each coefficient $\alpha \cdot s_{k}$ in (4.7) transforms as a section of the line bundle $\mathcal{O}_{S}(\Sigma) \otimes$ $K_{S}^{k}$. Even if $K_{S}$ itself has no holomorphic sections, such as occurs when $K_{S}$ is strictly negative (the del Pezzo case), the tensor product $\mathcal{O}_{S}(\Sigma) \otimes K_{S}^{k}$ might admit holomorphic sections, depending upon our choices for $\Sigma$ and $k$. Thus, we can further generalize (4.7) by taking

$$
\begin{aligned}
& y^{2}=x^{2}+\alpha z^{n+1}+\widehat{\alpha}_{2} z^{n-1}+\cdots+\widehat{\alpha}_{n+1}, \\
& \widehat{\alpha}_{k} \in H \frac{0}{\partial}\left(S, \mathcal{O}_{S}(\Sigma) \otimes K_{S}^{k}\right)
\end{aligned}
$$

where the $\widehat{\alpha}_{k}$ are the generalizations of the deformation parameters $\alpha_{k}$ given in (3.25) in the special case where $\alpha$ transforms as a section of a trivial bundle. As we discuss in subsection 4.3, the low-energy physics of F-theory on the local elliptic Calabi-Yau fourfold determined by (4.8) turns out to be quite rich, despite the fact that (4.8) describes the unfolding of only an $A_{n}$ singularity. In fact, from equation (4.7), we notice that the Calabi-Yau geometry remains regular even when the $s_{i}$ have first order poles along the locus $\alpha=0$. We will interpret this effect as the coupling of certain light modes living on $\Sigma$ which source the bulk field $\varphi$. In particular, the 
residue of $\varphi$ along the $\alpha=0$ pole will be identified with a condensate of these modes on $\Sigma$.

\section{Intersecting Seven-Branes of Arbitrary ADE-Type}

Since this paper is primarily concerned with exceptional singularities in F-theory, we must also consider intersecting seven-branes of arbitrary $A D E$-type. By analogy to the unfolding of the $A_{n}$ singularity over $S$ in (4.8), we thus consider local models for $X$ which are based on the unfolding of the general $A D E$-singularity.

In all cases, the essential feature of these models is that the coefficients corresponding to the analogues of the $\widehat{\alpha}_{k}$ in equation (4.8) are also holomorphic sections of appropriate line bundles over $S$. As these coefficients vary over $S$, the singularity along $S$ can enhance, at which point another seven-brane intersects the seven-brane wrapping $S$.

We now present our local models for $X$ which are based on the general unfolding of the $D$ - and $E$-type singularities. Again, some important numerology will be at play in the various line bundles which appear in the constructions below.

In the case that $X$ is associated to an unfolding of a $D$-type singularity, we take the coordinates $(x, y, z)$ on $Y$ to transform as sections of the rank three bundle

$$
V=\left(L \otimes K_{S}^{-1}\right) \oplus L \oplus K_{S}^{2}
$$

Here $L$ is an arbitrary line bundle over $S$. This ansatz for $V$ has been taken so that the equation for the $D_{n}$ singularity will be homogeneous in $(x, y, z)$ and so that the Calabi-Yau condition on $X$ will be satisfied.

With this ansatz for $V$, we then consider an equation for the hypersurface $Y$ of the form

$$
D_{n}: \quad y^{2}=-x^{2} z+\alpha^{2} z^{n-1}, \quad \alpha \in H \frac{0}{\partial}\left(S, L \otimes K_{S}^{-(n-1)}\right) .
$$

In this equation, $\alpha$ is an arbitrary holomorphic section of $L \otimes K_{S}^{-(n-1)}$ which we introduce as part of the defining data for the local Calabi-Yau fourfold $X$. If $L$ is sufficiently positive, then a non-trivial $\alpha$ always exists. Of course, we have made a special choice in assuming that $\alpha$ appears quadratically in (4.10). This choice is related to a 'splitness' condition for the singularity discussed in [31]. We will 
motivate our choice from the perspective of topological field theory later, but for now it is simply part of the model for $X$.

As in the preceding discussion of intersecting $A_{n}$ singularities, we let $\Sigma$ be the effective divisor in $S$ along which $\alpha$ vanishes, so that

$$
\mathcal{O}_{S}(\Sigma)=L \otimes K_{S}^{-(n-1)}
$$

Away from $\Sigma$, the equation in (4.10) thus describes a $D_{n}$ singularity over $S$. In precise analogy to (4.8), we now unfold the $D_{n}$ singularity in (4.10) as

$$
D_{n}: \quad y^{2}=-x^{2} z+\alpha^{2} z^{n-1}+\sum_{k=1}^{n-1} \widehat{\delta}_{2 k} z^{n-k-1}-2 \widehat{\gamma}_{n} x
$$

where:

$$
\widehat{\delta}_{2 k} \in H \frac{0}{\partial}\left(S, \mathcal{O}_{S}(2 \Sigma) \otimes K_{S}^{2 k}\right), \quad \widehat{\gamma}_{n} \in H_{\frac{0}{\partial}}\left(S, \mathcal{O}_{S}(\Sigma) \otimes K_{S}^{n}\right)
$$

Like $\alpha$, the sections $\widehat{\delta}_{2 k}$ and $\widehat{\gamma}_{n}$ are parameters which define the local model for $X$, and the line bundles appearing in (4.13) are just determined by homogeneity of (4.12).

Similarly, for the E-type singularities, we take $(x, y, z)$ to transform in the rank three bundle:

$$
V=L^{2} \oplus L^{3} \oplus\left(L \otimes K_{S}\right)
$$

where $L$ is again an arbitrary line bundle on $S$. As before, this ansatz for $V$ is fixed by homogeneity and the Calabi-Yau condition on $X$.

To describe a generic $E_{6,7,8}$ singularity over $S$, we consider the hypersurface equations for $Y$,

$$
\begin{array}{lll}
E_{6}: & y^{2}=x^{3}+\alpha^{2} z^{4} & \alpha \in H_{\bar{\partial}}^{0}\left(S, L \otimes K_{S}^{-2}\right) \\
E_{7}: & y^{2}=-x^{3}+16 \alpha x z^{3} & \alpha \in H_{\bar{\partial}}^{0}\left(S, L \otimes K_{S}^{-3}\right) \\
E_{8}: & y^{2}=x^{3}-\alpha z^{5} & \alpha \in H_{\bar{\partial}}^{0}\left(S, L \otimes K_{S}^{-5}\right) .
\end{array}
$$

As explained previously, the particular choice of numerical coefficients have been chosen for ease of comparison with the results of [28]. In each case, away from the divisor $\Sigma$ on which $\alpha$ vanishes, $Y$ has an exceptional singularity along $S$. In the case 
of the $E_{6}$ singularity, we have again made a special choice associated to the quadratic appearance of $\alpha$ in (4.15) and related to the splitness condition in [31].

We now unfold each exceptional singularity as:

$$
\begin{aligned}
E_{6}: \quad y^{2}= & x^{3}+\alpha^{2} z^{4}+\widehat{\varepsilon}_{2} x z^{2}+\widehat{\varepsilon}_{5} x z+\widehat{\varepsilon}_{6} z^{2}+\widehat{\varepsilon}_{8} x+\widehat{\varepsilon}_{9} z+\widehat{\varepsilon}_{12}, \\
E_{7}: \quad y^{2}= & -x^{3}+16 \alpha x z^{3}+\widehat{\varepsilon}_{2} x^{2} z+\widehat{\varepsilon}_{6} x^{2}+\widehat{\varepsilon}_{8} x z+ \\
& +\widehat{\varepsilon}_{10} z^{3}+\widehat{\varepsilon}_{12} x+\widehat{\varepsilon}_{14} z+\widehat{\varepsilon}_{18}, \\
E_{8}: \quad y^{2}= & x^{3}-\alpha z^{5}+\widehat{\varepsilon}_{2} x z^{3}+\widehat{\varepsilon}_{8} x z^{2}+\widehat{\varepsilon}_{12} z^{3}+\widehat{\varepsilon}_{14} x z+ \\
& +\widehat{\varepsilon}_{18} z^{2}+\widehat{\varepsilon}_{20} x+\widehat{\varepsilon}_{24} z+\widehat{\varepsilon}_{30} .
\end{aligned}
$$

Here the coefficients $\widehat{\varepsilon}_{k}$ transform as holomorphic sections of various line bundles which are determined by the homogeneity of the equations in (4.18). We collect the bundle assignments for these deformation parameters in the following tables:

\begin{tabular}{|l|l|l|l|}
\hline$E_{6}$ & & & \\
\hline & $\widehat{\varepsilon}_{2} \in \mathcal{O}_{S}(2 \Sigma) \otimes K_{S}^{2}$ & $\widehat{\varepsilon}_{5} \in \mathcal{O}_{S}(3 \Sigma) \otimes K_{S}^{5}$ & $\widehat{\varepsilon}_{6} \in \mathcal{O}_{S}(4 \Sigma) \otimes K_{S}^{6}$ \\
\hline & $\widehat{\varepsilon}_{8} \in \mathcal{O}_{S}(4 \Sigma) \otimes K_{S}^{8}$ & $\widehat{\varepsilon}_{9} \in \mathcal{O}_{S}(5 \Sigma) \otimes K_{S}^{9}$ & $\widehat{\varepsilon}_{12} \in \mathcal{O}_{S}(6 \Sigma) \otimes K_{S}^{12}$ \\
\hline
\end{tabular}

\begin{tabular}{|l|l|l|l|}
\hline$E_{7}$ & & & \\
\hline & $\widehat{\varepsilon}_{2} \in \mathcal{O}_{S}(\Sigma) \otimes K_{S}^{2}$ & $\widehat{\varepsilon}_{6} \in \mathcal{O}_{S}(2 \Sigma) \otimes K_{S}^{6}$ & $\widehat{\varepsilon}_{8} \in \mathcal{O}_{S}(3 \Sigma) \otimes K_{S}^{8}$ \\
\hline & $\widehat{\varepsilon}_{10} \in \mathcal{O}_{S}(3 \Sigma) \otimes K_{S}^{10}$ & $\widehat{\varepsilon}_{12} \in \mathcal{O}_{S}(4 \Sigma) \otimes K_{S}^{12}$ & $\widehat{\varepsilon}_{14} \in \mathcal{O}_{S}(5 \Sigma) \otimes K_{S}^{14}$ \\
\hline & $\widehat{\varepsilon}_{18} \in \mathcal{O}_{S}(6 \Sigma) \otimes K_{S}^{18}$ & & \\
\hline
\end{tabular}

\begin{tabular}{|l|l|l|l|}
\hline$E_{8}$ & & & \\
\hline & $\widehat{\varepsilon}_{2} \in \mathcal{O}_{S}(\Sigma) \otimes K_{S}^{2}$ & $\widehat{\varepsilon}_{8} \in \mathcal{O}_{S}(2 \Sigma) \otimes K_{S}^{8}$ & $\widehat{\varepsilon}_{12} \in \mathcal{O}_{S}(3 \Sigma) \otimes K_{S}^{12}$ \\
\hline & $\widehat{\varepsilon}_{14} \in \mathcal{O}_{S}(3 \Sigma) \otimes K_{S}^{14}$ & $\widehat{\varepsilon}_{18} \in \mathcal{O}_{S}(3 \Sigma) \otimes K_{S}^{18}$ & $\widehat{\varepsilon}_{20} \in \mathcal{O}_{S}(4 \Sigma) \otimes K_{S}^{20}$ \\
\hline & $\widehat{\varepsilon}_{24} \in \mathcal{O}_{S}(5 \Sigma) \otimes K_{S}^{24}$ & $\widehat{\varepsilon}_{30} \in \mathcal{O}_{S}(6 \Sigma) \otimes K_{S}^{30}$ & \\
\hline
\end{tabular}

A non-trivial feature of the above bundle assignments is that the power of $K_{S}$ ap- 
pearing in each entry again precisely matches to the degrees of the primitive Casimir invariant. The discrepancy by an overall factor of $\mathcal{O}_{S}(n \Sigma)$ anticipates the beautiful match we shall find between condensates of fields localized on $\Sigma$ and the geometric unfolding of the singularity.

The class of local, elliptic Calabi-Yau fourfolds described by equations (4.8), (4.12), and (4.18) is quite broad, and serves as a significant extension over the very restricted class of models studied in Section 3. Given such a local Calabi-Yau fourfold $X$, one of our primary goals in the rest of the paper will be to determine the lowenergy effective description for F-theory compactified on $X$.

\subsection{Topological Field Theory on a Defect}

The local geometries for the elliptically-fibered Calabi-Yau fourfold $X$ introduced in Section 4.1 correspond in F-theory to rather complicated configurations of intersecting seven-branes inside the threefold $B$ sitting at the base of $X$. So if we wish to analyze F-theory on $X$, we clearly need to know something about the low-energy effective description for intersecting seven-branes in F-theory.

For concreteness, let us consider two seven-branes wrapping smooth complex surfaces $S$ and $S^{\prime}$ in $B$ such that $S$ and $S^{\prime}$ intersect transversely along a complex curve $\Sigma=S \cap S^{\prime}$. In general, $\Sigma$ might be reducible and consist of several components which themselves intersect at points inside $S$. However, to keep matters simple, we suppose that $\Sigma$ is an irreducible, smooth curve. In Section 5, we consider what happens when $\Sigma$ becomes reducible.

As explained in Section 3, on each of $S$ and $S^{\prime}$ is a twisted Yang-Mills theory which captures the effective dynamics for the low-energy degrees of freedom living on a seven-brane in F-theory. But just as for ordinary D7-branes, we now expect additional light degrees of freedom to be localized along the subspace $\mathbb{R}^{3,1} \times \Sigma$ where the seven-branes wrapping $S$ and $S^{\prime}$ intersect. These light degrees of freedom on $\mathbb{R}^{3,1} \times \Sigma$ are then described by an effective defect theory coupled to the bulk YangMills theories on $\mathbb{R}^{3,1} \times S$ and $\mathbb{R}^{3,1} \times S^{\prime}$.

If the defect theory on $\mathbb{R}^{3,1} \times \Sigma$ is to preserve $\mathcal{N}=1$ supersymmetry in four dimensions, the defect theory must be twisted along $\Sigma$ for the same reason that the bulk Yang-Mills theory on $\mathbb{R}^{3,1} \times S$ is twisted along $S$. Though the corresponding defect theories that live on the intersections of D-branes in Minkowski and Anti-de 
Sitter space have been studied for example in [32, 33, 34], little attention has been paid to their twisted relatives. For this reason, we now establish some basic facts about the structure of the partially twisted theory on $\mathbb{R}^{3,1} \times \Sigma$.

\subsubsection{Topological Twist on $\Sigma$}

We first identify the light defect degrees of freedom that propagate on $\mathbb{R}^{3,1} \times \Sigma$. The simplest case to consider is $\Sigma=\mathbb{C}$, corresponding to F-theory compactified to $\mathbb{R}^{5,1}$ on an elliptically-fibered Calabi-Yau threefold. Such an F-theory background preserves $\mathcal{N}=2$ supersymmetry in four dimensions, and the massless charged matter arising from intersecting seven-branes in that situation was analyzed in [22].

To recall the result of [22], we suppose that the seven-brane wrapping $S$ carries a worldvolume gauge group $G_{S}$, and the seven-brane wrapping $S^{\prime}$ carries a worldvolume gauge group $G_{S^{\prime}}$. Both $G_{S}$ and $G_{S^{\prime}}$ are simply-laced Lie groups associated to $A D E$ singularities along $S$ and $S^{\prime}$. We also allow the possibility that either $G_{S}$ or $G_{S^{\prime}}$ is $U(1)$, corresponding to a Kodaira fiber of type $\mathrm{I}_{1}$ over $S$ or $S^{\prime}$. At the intersection of $S$ and $S^{\prime}$ over $\Sigma$, the singularities generically enhance to a new singularity associated to a simply-laced Lie group $G_{\Sigma}$, where

$$
G_{S} \times G_{S^{\prime}} \subset G_{\Sigma}
$$

According to [22], a massless hypermultiplet charged under $G_{S} \times G_{S^{\prime}}$ then propagates along the seven-brane intersection.

In the case $G_{S}=S U(n+1), G_{S^{\prime}}=S U(m+1)$, and $G_{\Sigma}=S U(m+n+2)$, the charged hypermultiplet decomposes into a pair of $\mathcal{N}=1$ chiral multiplets which transform as bifundamentals of $G_{S} \times G_{S^{\prime}}$. To extend the notion of "bifundamental" matter to the general $A D E$ setting, we decompose the adjoint representation of $G_{\Sigma}$ under $G_{S} \times G_{S^{\prime}}$ as

$$
\operatorname{ad}\left(G_{\Sigma}\right)=\operatorname{ad}\left(G_{S}\right) \oplus \operatorname{ad}\left(G_{S^{\prime}}\right) \oplus\left[\bigoplus_{j} U_{j} \otimes U_{j}^{\prime}\right]
$$

Here $U_{j}$ and $U_{j}^{\prime}$ are irreducible representations of $G_{S}$ and $G_{S^{\prime}}$, and ' $j$ ' is a dummy index running over whatever summands appear in the decomposition above. Under the decomposition of the hypermultiplet into $\mathcal{N}=1$ chiral multiplets, the light 
"bifundamental" matter localized along $\Sigma$ then transforms in the representation of $G_{S} \times G_{S^{\prime}}$ given by the non-adjoint summand of (4.23), namely

$$
\bigoplus_{j} U_{j} \otimes U_{j}^{\prime}
$$

If $\Sigma$ is not $\mathbb{C}$ but rather a compact complex curve, we apply the adiabatic argument as in Section 3 to conclude that the massless fields which propagate on $\mathbb{R}^{3,1} \times \Sigma$ correspond to a twisted version of the charged hypermultiplet propagating on $\mathbb{R}^{5,1}$. Once again, the requirement of $\mathcal{N}=1$ supersymmetry in four dimensions leaves us with no choice about how to twist the hypermultiplet.

To specify the twist, we recall that the untwisted hypermultiplet on $\mathbb{R}^{5,1}$ contains a pair of complex bosons $\left(\sigma, \bar{\sigma}^{c}\right)$ and a negative-chirality ${ }^{18}$ Weyl fermion, which transforms in the $4^{\prime}$ of $S O(5,1)$. Under the gauge group $G_{S} \times G_{S^{\prime}}$ in $(4.22)$, we take the bosons and fermions in the hypermultiplet to transform in the representation $U \otimes U^{\prime}$, corresponding to one of the summands in (4.24). If all kinetic terms are canonical, the hypermultiplet also respects a global $S U(2)_{R}$ symmetry under which $\left(\sigma, \bar{\sigma}^{c}\right)$ transform as a doublet, and the Weyl fermion transforms as a singlet. Finally, the supersymmetry generator $\epsilon$ transforms under $S O(5,1) \times S U(2)_{R}$ in the representation $\mathbf{4}^{\prime} \otimes \mathbf{2 .}^{19}$

To twist the hypermultiplet on $\Sigma$, we reduce the global $S O(5,1)$ symmetry to $S O(3,1) \times U(1)$, where the $U(1)$ factor is to be identified with the structure group of the tangent bundle on $\Sigma$. Under the reduction to $S O(3,1) \times U(1)$, the negativechirality spinor of $S O(5,1)$ decomposes as

$$
4^{\prime} \longmapsto\left(2,1,-\frac{1}{2}\right) \oplus\left(\mathbf{1}, \mathbf{2},+\frac{1}{2}\right) .
$$

To specify the bundle assignments of the twisted theory, note that the $\mathbf{2}$ of $S U(2)_{R}$ decomposes to the Cartan $U(1)_{R}$ subgroup as:

$$
\mathbf{2} \longmapsto \mathbf{1}_{+1} \oplus \mathbf{1}_{-1}
$$

\footnotetext{
${ }^{18}$ Our choice of chirality here may seem a bit strange, but it makes certain conventions about holomorphy more natural later.

${ }^{19}$ The generator $\epsilon$ also obeys a reality condition which will play no role in the discussion to follow.
} 
The subscripts above indicate the charges under the generator $R$ of $U(1)_{R}$. Equivalently, the distinguished $U(1)_{R}$ subgroup of $S U(2)_{R}$ can be identified with the R-symmetry used to partially twist the bulk eight-dimensional Yang-Mills theory described in Section 3.

The twist of the hypermultiplet is now specified by a homomorphism from $U(1)_{R}$ to the $U(1)$ factor in $S O(3,1) \times U(1)$. Let $J$ be the generator of the $U(1)$ factor in $S O(3,1) \times U(1)$ normalized according to (4.25). In order to preserve $\mathcal{N}=1$ supersymmetry on $\mathbb{R}^{3,1}$, half of the original eight supersymmetries generated by $\epsilon$ must transform as scalars on $\Sigma$ once we twist. As one can check, this requirement implies that the generator $J_{t o p}$ of the twisted $U(1)$ must be

$$
J_{\text {top }}=J \pm \frac{1}{2} R .
$$

Either choice of sign above leads to an isomorphic twist, so we take $J_{\text {top }}=J-\frac{1}{2} R$ without loss of generality.

Because the fermions in the hypermultiplet transform trivially under $S U(2)_{R}$, the twist by $U(1)_{R} \subset S U(2)_{R}$ does not alter their geometric interpretation on $\Sigma$. According to (4.25), the twisted hypermultiplet on $\mathbb{R}^{3,1} \times \Sigma$ therefore contains fermions $\left(\lambda_{\alpha}, \bar{\lambda}_{\dot{\alpha}}^{c}\right)$ transforming as spinors on the curve $\Sigma$,

$$
\begin{array}{ll}
\lambda_{\alpha} & \text { section of } K_{\Sigma}^{1 / 2} \otimes \mathscr{U} \otimes \mathscr{U}^{\prime}, \\
\bar{\lambda}_{\dot{\alpha}}^{c} & \text { section of } \bar{K}_{\Sigma}^{1 / 2} \otimes \mathscr{U} \otimes \mathscr{U}^{\prime} .
\end{array}
$$

In (4.28), $K_{\Sigma}^{1 / 2}$ denotes a square-root of the canonical bundle $K_{\Sigma}$ on $\Sigma$. In general, the choice of a square-root for $K_{\Sigma}$ is not unique. If $\Sigma$ has genus $g$, then $\Sigma$ admits $2^{2 g}$ distinct spin structures, and we must identify precisely which one we pick to define $K_{\Sigma}^{1 / 2}$. As we explain in Section 4.2.3, $\Sigma$ inherits a distinguished spin structure from its embedding in the surface $S$ (or equally well from its embedding in $S^{\prime}$ ), and the distinguished spin structure defines $K_{\Sigma}^{1 / 2}$. Also, $\mathscr{U}$ and $\mathscr{U}^{\prime}$ are vector bundles on $\Sigma$ associated to the representations $U$ and $U^{\prime}$. As one expects, $\mathscr{U}$ and $\mathscr{U}^{\prime}$ are determined by the restriction to $\Sigma$ of principal bundles $P$ and $P^{\prime}$ over the respective surfaces $S$ and $S^{\prime}$.

Even though the fermions in the hypermultiplet are not affected by the twist on $\Sigma$, the bosons in the hypermultiplet are. Under the generator $J_{\text {top }}, \sigma$ and $\bar{\sigma}^{c}$ carry 
respective charges $\mp \frac{1}{2}$, inherited from their charges under $U(1)_{R}$. As a result, $\sigma$ and $\bar{\sigma}^{c}$ also transform as spinors on $\Sigma$,

$$
\begin{array}{ll}
\sigma & \text { section of } K_{\Sigma}^{1 / 2} \otimes \mathscr{U} \otimes \mathscr{U}^{\prime}, \\
\bar{\sigma}^{c} & \text { section of } \bar{K}_{\Sigma}^{1 / 2} \otimes \mathscr{U} \otimes \mathscr{U}^{\prime} .
\end{array}
$$

Finally, as is often useful when we discuss the hypermultiplet in the language of $\mathcal{N}=1$ supersymmetry, we introduce the complex boson $\sigma^{c}$ and the fermion $\lambda_{\alpha}^{c}$ which are the CPT conjugates of $\bar{\sigma}^{c}$ and $\bar{\lambda}_{\dot{\alpha}}^{c}$,

$$
\begin{array}{ll}
\sigma^{c} & \text { section of } K_{\Sigma}^{1 / 2} \otimes \mathscr{U}^{*} \otimes\left(\mathscr{U}^{\prime}\right)^{*}, \\
\lambda_{\alpha}^{c} & \text { section of } K_{\Sigma}^{1 / 2} \otimes \mathscr{U}^{*} \otimes\left(\mathscr{U}^{\prime}\right)^{*} .
\end{array}
$$

Here $\mathscr{U}^{*}$ and $\left(\mathscr{U}^{\prime}\right)^{*}$ are the bundles associated to the dual representations $U^{*}$ and $\left(U^{\prime}\right)^{*}$. Of course, if $U \otimes U^{\prime}$ appears as a summand in (4.24), then so too does $U^{*} \otimes\left(U^{\prime}\right)^{*}$, since the representation in (4.24) is necessarily real.

\subsubsection{On the Supersymmetric Defect Action}

We are still left to determine the supersymmetric action for the twisted defect theory on $\mathbb{R}^{3,1} \times S$. In particular, we need to consider how the defect fields on $\Sigma$ couple to the bulk Yang-Mills fields on $S$ and $S^{\prime}$. As will be clear, supersymmetry and gauge-invariance leave us little choice as to how the coupling can be done.

Like the partially twisted Yang-Mills theory discussed in Section 3, the twisted fields in the hypermultiplet on $\mathbb{R}^{3,1} \times \Sigma$ naturally arrange themselves into the standard representations of the $\mathcal{N}=1$ supersymmetry algebra on $\mathbb{R}^{3,1}$. Thus from the field content in (4.28), (4.29), and (4.30), we clearly obtain $\mathcal{N}=1$ chiral multiplets

$$
\left(\sigma, \lambda_{\alpha}\right), \quad\left(\sigma^{c}, \lambda_{\alpha}^{c}\right)
$$

along with the CPT-conjugate $\mathcal{N}=1$ anti-chiral multiplets, which will not play a role in the following.

We are now in a slightly unusual situation. In the most common topological field theories, the on-shell supersymmetries of the ten-dimensional Yang-Mills theory immediately determine the on-shell supersymmetries of the lower-dimensional 
twisted theory. From the on-shell twisted supersymmetries, one then tries to write an appropriately twisted version of the super Yang-Mills action.

But in the case at hand, we do not know a priori how the on-shell supersymmetries should act on the chiral multiplets in (4.31), since we do not yet know precisely how the defect theory will couple to the bulk Yang-Mills theories on $S$ and $S^{\prime}$. However, we do have an (essentially) off-shell formulation for the $\mathcal{N}=1$ supersymmetry algebra in our problem. Inverting the usual order of analysis, in Appendix $\mathrm{D}$ we therefore construct the most general supersymmetric off-shell action for the six-dimensional defect theory. We then integrate out auxiliary fields to determine the on-shell supersymmetry transformations and associated BPS equations for the system. The details of the analysis in Appendix D are mostly unimportant, but the analysis does yield two important results.

\section{The Defect Superpotential}

First, the off-shell defect action, including couplings to the bulk Yang-Mills fields on $S$ and $S^{\prime}$, can be written concisely in superspace as

$$
I_{\Sigma}=\int_{\mathbb{R}^{3,1} \times \Sigma} d^{4} x d^{2} \bar{\theta} \mathcal{O}+\int_{\mathbb{R}^{3,1} \times \Sigma} d^{4} x d^{2} \theta W_{\Sigma},
$$

where

$$
W_{\Sigma}=\left\langle\boldsymbol{\Lambda}^{c}, \bar{\partial}_{\mathbf{A}+\mathbf{A}^{\prime}} \boldsymbol{\Lambda}\right\rangle
$$

The notation of the above expression is explained below. In $(4.32), \mathcal{O}$ is a gaugeinvariant operator whose particular form does not much matter once we restrict attention to the cohomology of $\bar{Q}_{\dot{\alpha}}$. As we demonstrate in Appendix D, the terms in $I_{\Sigma}$ derived from $\mathcal{O}$ are the standard kinetic terms on $\mathbb{R}^{3,1}$ for the defect fields, along with couplings conjugate to those derived from the superpotential in (4.32).

The superpotential $W_{\Sigma}$ is clearly the object of interest in $I_{\Sigma}$. To explain our notation, $\boldsymbol{\Lambda}$ and $\boldsymbol{\Lambda}^{c}$ are chiral superfields associated to the chiral multiplets in (4.31), so that

$$
\begin{aligned}
\boldsymbol{\Lambda} & =\sigma+\sqrt{2} \theta \lambda+\theta \theta \mathcal{K}+\cdots, \\
\boldsymbol{\Lambda}^{c} & =\sigma^{c}+\sqrt{2} \theta \lambda^{c}+\theta \theta \mathcal{K}^{c}+\cdots .
\end{aligned}
$$


Here $\mathcal{K}$ and $\mathcal{K}^{c}$ are auxiliary bosonic fields that transform like $\sigma$ and $\sigma^{c}$ as sections of $K_{\Sigma}^{1 / 2} \otimes \mathscr{U} \otimes \mathscr{U}^{\prime}$ and $K_{\Sigma}^{1 / 2} \otimes \mathscr{U}^{*} \otimes\left(\mathscr{U}^{\prime}\right)^{*}$, and the '...' indicate additional terms involving $\bar{\theta}^{\dot{\alpha}}$ which do not play a role in our discussion of $W_{\Sigma}$. The pairing $\langle\cdot, \cdot\rangle$ between $\Lambda$ and $\Lambda^{c}$ in $W_{\Sigma}$ is the canonical pairing between sections of $\mathscr{U} \otimes \mathscr{U}^{\prime}$ and the dual. Recall that from the four-dimensional perspective, the $(0,1)$ component of the gauge field along $S$ appears as the lowest bosonic component of the chiral superfield described in Section 3,

$$
\mathbf{A}_{\bar{m}}=A_{\bar{m}}+\sqrt{2} \theta \psi_{\bar{m}}+\theta \theta \mathcal{G}_{\bar{m}}+\cdots
$$

where $\mathcal{G}_{\bar{m}}$ is an auxiliary bosonic field transforming on $S$ as a section of $\bar{\Omega}_{S}^{1} \otimes \operatorname{ad}(P)$. Finally, the covariant derivative $\bar{\partial}_{\mathbf{A}+\mathbf{A}^{\prime}}$ appearing in (4.32) is meant to be interpreted literally in superspace as

$$
\begin{aligned}
\bar{\partial}_{\mathbf{A}+\mathbf{A}^{\prime}} & =\bar{\partial}+\mathbf{A}+\mathbf{A}^{\prime}, \\
& =\bar{\partial}_{A+A^{\prime}}+\sqrt{2} \theta\left(\psi+\psi^{\prime}\right)+\theta \theta\left(\mathcal{G}+\mathcal{G}^{\prime}\right)+\cdots
\end{aligned}
$$

Given (4.34), (4.35), and (4.36), we can immediately work out the component expansion for the superpotential in $I_{\Sigma}$. However, before we do so, let us note that $W_{\Sigma}$ is quite special. Due to the twisting of $\boldsymbol{\Lambda}$ and $\boldsymbol{\Lambda}^{c}$ as spinors on $\Sigma$, the superpotential in (4.33) itself transforms as a differential form of type $(1,1)$ on $\Sigma$. Hence $W_{\Sigma}$ can be naturally integrated over $\Sigma$, without reference to a metric on the curve. As a result, when we compactify the defect theory to $\mathbb{R}^{3,1}$ in Section $4.4, W_{\Sigma}$ leads immediately to effective Yukawa couplings in four dimensions which involve only holomorphic data on $\Sigma$.

\section{BPS Equations}

The second important result obtained in Appendix D is a derivation of the conditions for unbroken supersymmetry when the defect theory on $\mathbb{R}^{3,1} \times \Sigma$ is coupled to the bulk Yang-Mills theories on $\mathbb{R}^{3,1} \times S$ and $\mathbb{R}^{3,1} \times S^{\prime}$ via the action in (4.32).

From the perspective of the present paper, the most interesting BPS equations are the F-term equations associated to the auxiliary fields which enter $W_{\Sigma}$. Explicitly, 
we expand in components

$$
\begin{aligned}
\int_{\mathbb{R}^{3,1} \times \Sigma} d^{4} x d^{2} \theta W_{\Sigma}= & \int_{\mathbb{R}^{3,1} \times \Sigma} d^{4} x\left[\left\langle\mathcal{K}^{c}, \bar{\partial}_{A+A^{\prime}} \sigma\right\rangle+\left\langle\sigma^{c}, \bar{\partial}_{A+A^{\prime}} \mathcal{K}\right\rangle+\left\langle\sigma^{c},\left(\mathcal{G}+\mathcal{G}^{\prime}\right) \cdot \sigma\right\rangle\right. \\
& \left.-\left\langle\lambda^{c}, \bar{\partial}_{A+A^{\prime}} \lambda\right\rangle-\left\langle\sigma^{c},\left(\psi+\psi^{\prime}\right) \cdot \lambda\right\rangle-\left\langle\lambda^{c},\left(\psi+\psi^{\prime}\right) \cdot \sigma\right\rangle\right] .
\end{aligned}
$$

Here in expressions such as $\mathcal{G} \cdot \sigma$, we indicate the linear action by elements in the Lie algebra of the group $G_{S}$ on the representation $U$, and similarly for the action of $G_{S^{\prime}}$ on the representation $U^{\prime}$.

Because $\mathcal{K}$ and $\mathcal{K}^{c}$ are the auxiliary bosonic fields appearing in the defect chiral superfields $\boldsymbol{\Lambda}$ and $\boldsymbol{\Lambda}^{c}$, the linear terms involving $\mathcal{K}$ and $\mathcal{K}^{c}$ in (4.37) immediately imply the F-term supersymmetry conditions

$$
\bar{\partial}_{A+A^{\prime}} \sigma=\bar{\partial}_{A+A^{\prime}} \sigma^{c}=0
$$

Thus $\sigma$ and $\sigma^{c}$ must be holomorphic as sections of the respective bundles $K_{\Sigma}^{1 / 2} \otimes$ $\mathscr{U} \otimes \mathscr{U}^{\prime}$ and $K_{\Sigma}^{1 / 2} \otimes \mathscr{U}^{*} \otimes\left(\mathscr{U}^{\prime}\right)^{*}$ on $\Sigma$.

The essentially new ingredient in (4.37) is the coupling of the auxiliary bulk fields $\mathcal{G}$ and $\mathcal{G}^{\prime}$ appearing in $\mathbf{A}$ and $\mathbf{A}^{\prime}$ to the defect fields $\sigma$ and $\sigma^{c}$. In the absence of the defect, the F-term supersymmetry condition associated to $\mathcal{G}$ (and similarly to $\mathcal{G}^{\prime}$ ) merely states that

$$
\bar{\partial}_{A} \varphi=0
$$

so that $\varphi$ is holomorphic as a section of $K_{S} \otimes \operatorname{ad}(P)$ on $S$.

However, in the presence of the defect, the linear coupling to $\mathcal{G}$ in equation (4.37) induces a source term for the BPS equation in (4.39), so that the new supersymmetry condition on $\varphi$ becomes

$$
\bar{\partial}_{A} \varphi=\delta_{\Sigma}\left\langle\left\langle\sigma^{c}, \sigma\right\rangle\right\rangle_{\operatorname{ad}(P)} .
$$

Here $\delta_{\Sigma}$ is a two-form on $S$ with delta-function support along $\Sigma$ which represents the Poincaré dual of $\Sigma$. In particular, because $\Sigma$ is a holomorphic curve, $\delta_{\Sigma}$ has holomorphic/anti-holomorphic type $(1,1)$ on $S$, so that both sides of $(4.40)$ are associated to differential forms of type $(2,1)$ on $S$. 
We also introduce in (4.40) the natural 'outer-product' determined by the action of $G$ on $U$,

$$
\langle\langle\cdot, \cdot\rangle\rangle_{\operatorname{ad}(P)}:\left.\left[\mathscr{U}^{*} \otimes\left(\mathscr{U}^{\prime}\right)^{*}\right] \otimes\left[\mathscr{U} \otimes \mathscr{U}^{\prime}\right] \longrightarrow \operatorname{ad}(P)\right|_{\Sigma},
$$

obtained from the individual pairings

$$
\left.\mathscr{U}^{*} \otimes \mathscr{U} \longrightarrow \operatorname{ad}(P)\right|_{\Sigma}, \quad\left(\mathscr{U}^{\prime}\right)^{*} \otimes \mathscr{U}^{\prime} \longrightarrow \mathcal{O}_{\Sigma}
$$

Explicitly, if $\left(T^{I}\right)_{a^{\prime}}^{a}$ for $I=1, \ldots, \operatorname{dim}\left(G_{S}\right)$ represent the generators of $G_{S}$ acting on $U$ in a given basis $\left\{u^{a}\right\}$, then locally $\left\langle\left\langle\sigma^{c}, \sigma\right\rangle\right\rangle_{\operatorname{ad}(P)}=\sigma_{a}^{c}\left(T^{I}\right)_{a^{\prime}}^{a} \sigma^{a^{\prime}}$.

As will be very important in Section 4.3, configurations of $\varphi$ which satisfy equation (4.40) have a very natural interpretation in algebraic geometry. Namely, the deltafunction source appearing on the right in equation (4.40) implies that $\varphi$ is now a meromorphic section of $K_{S} \otimes \operatorname{ad}(P)$ which has a first-order pole along $\Sigma$, with residue given by $\left\langle\left\langle\sigma^{c}, \sigma\right\rangle\right\rangle_{\operatorname{ad}(P)}$. Thus, if $\Sigma$ is determined by the vanishing of $\alpha$ as in Section 4.1 , then $\varphi$ appears locally near $\Sigma$ as

$$
\varphi=\frac{\left\langle\left\langle\sigma^{c}, \sigma\right\rangle\right\rangle_{\mathrm{ad}(P)} d s^{1} \wedge d s^{2}}{\alpha}+\cdots,
$$

where $\left(s^{1}, s^{2}\right)$ are local holomorphic coordinates near $\Sigma \subset S$, and the '...' indicate terms which are regular in $\varphi$.

We will have quite a bit more to say about equation (4.40) in Section 4.3, but for now let us record the rest of the BPS equations in the coupled defect and Yang-Mills theories. The presence of the defect on $\Sigma$ does not change the conditions

$$
F_{S}^{(0,2)}=F_{S}^{(2,0)}=0
$$

Thus the gauge field on $S$ still endows the principal $G_{S}$-bundle $P$ with a holomorphic structure in the presence of the defect. However, the defect does induce source terms in the D-term equation for the gauge field, so that the new D-term equation becomes

$$
\omega \wedge F_{S}+\frac{i}{2}[\varphi, \bar{\varphi}]=\frac{1}{2} \omega \wedge \delta_{\Sigma}\left[\mu(\bar{\sigma}, \sigma)-\mu\left(\bar{\sigma}^{c}, \sigma^{c}\right)\right]
$$

In (4.45), we have introduced the moment map $\mu$ associated to the action of $G_{S}$ 
on the representation $U$. Thus,

$$
\mu(\cdot, \cdot):\left.\left[\overline{\mathscr{U}} \otimes \overline{\mathscr{U}^{\prime}}\right] \otimes\left[\mathscr{U} \otimes \mathscr{U}^{\prime}\right] \longrightarrow \operatorname{ad}(P)\right|_{\Sigma}
$$

and dually when $\mu$ is evaluated on $\mathscr{U}^{*} \otimes\left(\mathscr{U}^{\prime}\right)^{*}$. The moment map $\mu$ is closely related to the canonical outer-product $\langle\langle\cdot, \cdot\rangle\rangle_{\mathrm{ad}(P)}$ that we introduced earlier, but $\mu$ is defined using a hermitian metric on the bundles $\mathscr{U}$ and $\mathscr{U}^{\prime}$. This hermitian metric is also used to define the kinetric terms on $\mathbb{R}^{3,1}$ for $\sigma$ and $\sigma^{c}$, as in Appendix D. Explicitly, in terms of the local generators $\left(T^{I}\right)_{a^{\prime}}^{a}$ which we introduced to describe the outer-product $\langle\langle\cdot, \cdot\rangle\rangle_{\mathrm{ad}(P)}$, the moment map is given as usual by $\mu(\bar{\sigma}, \sigma)=\bar{\sigma}^{\bar{a}}\left(T^{I}\right)_{\bar{a} a} \sigma^{a}$.

Finally, for each BPS equation involving the gauge field $A$ or scalar $\varphi$ in (4.40), (4.44), and (4.45), we obtain completely parallel BPS equations for the gauge field $A^{\prime}$ and the scalar $\varphi^{\prime}$ on the surface $S^{\prime}$.

\subsubsection{The Defect as a Cosmic String}

So far, we have used indirect arguments to determine the structure of the defect theory on $\mathbb{R}^{3,1} \times \Sigma$. Those arguments were based upon the adiabatic extension of older ideas [22] about the charged matter present in F-theory compactifications on Calabi-Yau threefolds, along with the requirement of $\mathcal{N}=1$ supersymmetry in four dimensions. However, with a bit more work, the structure of the defect theory on $\mathbb{R}^{3,1} \times \Sigma$ can also be determined directly from the partially twisted Yang-Mills theory on $\mathbb{R}^{3,1} \times S$ itself. Furthermore, as we explain, the analysis from the perspective of $S$ identifies the proper spin structure on $\Sigma$ with which to define the bundle $K_{\Sigma}^{1 / 2}$.

The analysis we are about to perform is not really new. In the context of F-theory compactifications on Calabi-Yau threefolds, precisely the same arguments were originally used in [22] to deduce the presence of charged hypermultiplets on $\mathbb{R}^{5,1}$, a result which is the basic ingredient in our previous adiabatic analysis. Essentially the same ideas have also appeared in [35], where a twisted version of four-dimensional, $\mathcal{N}=1$ supersymmetric Yang-Mills theory on a Kähler manifold is considered. Nonetheless, the following observations do serve to illuminate the relationship between the bulk Yang-Mills theory on $S$ and the defect theory on $\Sigma$, and therefore they seem worthwhile to review. 
To start, let us assume that the holomorphic surface $S$ satisfies $h^{2,0}(S) \neq 0$, so that $K_{S}$ admits holomorphic sections and $S \subset B$ is not rigid. In this case, if we start with a seven-brane with worldvolume gauge group $G_{S}$ which wraps $S$, then to obtain a configuration of intersecting seven-branes in $B$, we can simply turn on a holomorphic expectation value for the twisted scalar field $\varphi$.

Specifically, we assume that $\varphi$ takes the abelian form

$$
\varphi=\varphi_{0} t, \quad \varphi_{0} \in H_{\bar{\partial}}^{0}\left(S, K_{S}\right), \quad t \in \operatorname{ad}\left(G_{S}\right) .
$$

Here $\varphi_{0}$ is a non-trivial element in $H \frac{0}{\partial}\left(S, K_{S}\right)$, and $t$ is a fixed generator in the Lie algebra of $G_{S}$. In this very simple background, we also take the connection on $P$ to be trivial, so that $F_{S}=0$ and the D-term equation on $S$ is automatically satisfied.

Simple though the background described by (4.47) may be, it still describes a configuration of intersecting seven-branes in $B$. The expectation value in (4.47) generically breaks the simply-laced group $G_{S}$ to a product

$$
\Gamma_{S} \times U(1) \subset G_{S},
$$

where the $U(1)$ factor is generated by $t$, and $\Gamma_{S}$ is generated by the other elements in the Lie algebra of $G_{S}$ which commute with $t$. Equivalently, from the perspective of the local Calabi-Yau fourfold $X$ introduced in Section 3 to describe the original seven-brane wrapping $\mathbb{R}^{3,1} \times S$ with gauge group $G_{S}$, the expectation value for $\varphi$ determines a deformation of $X$ as in (3.25), such that the generic singularity along $S$ unfolds to the singularity corresponding to the simply-laced group $\Gamma_{S}$. Finally, from the physical perspective, the expectation value for $\varphi$ implies that some seven-branes have been moved away from the original stack of seven-branes wrapping $S$, such that the gauge group $G_{S}$ is generically broken as in (4.48).

The qualifier "generic" in the preceding statement is essential. Unless $K_{S}$ is trivial, $\varphi$ vanishes over a curve $\Sigma \subset S$ representing the canonical divisor of $S$. At points in $\Sigma$ where $\varphi$ vanishes, the gauge group $G_{S}$ is unbroken, the generic singularity along $S$ enhances, and the seven-brane represented by the $U(1)$ factor in (4.48) intersects the other seven-branes associated to the generic singularity of type $\Gamma_{S}$ along $S$. Hence a configuration of intersecting seven-branes with respective worldvolume gauge groups $\Gamma_{S}$ and $U(1)$ can be described purely in terms of the twisted Yang-Mills theory on $\mathbb{R}^{3,1} \times S$ with gauge group $G_{S}$, such that $\varphi$ has the expectation value in 
(4.47).

To determine the structure of the defect theory along $\Sigma$, we therefore want to consider what happens in the eight-dimensional Yang-Mills theory when $\varphi$ has a nontrivial, holomorphic expectation value. Certainly away from the curve $\Sigma \subset S$ where $\varphi$ vanishes, the usual Higgs mechanism operates, and the bifundamental components of the Yang-Mills multiplet on $S$ are massive. Here we use "bifundamental" in the general sense that it is defined in (4.23) and (4.24).

Over $\Sigma$, something more interesting happens. As discussed in [35], $\Sigma$ appears from the four-dimensional perspective of $S$ as a kind of global cosmic string associated to the vanishing of the holomorphic mass term induced by $\varphi$ for the bifundamental components of the Yang-Mills multiplet. In precisely this situation, one expects to find bifundamental boson and fermion zero-modes which are trapped along $\Sigma$ and which lead to massless, charged matter on $\mathbb{R}^{3,1}$.

We naturally want to identify the massless modes trapped along $\Sigma$ with the defect degrees of freedom that we initially introduced by hand on $\Sigma$. To do so, let us identify which fermion zero-modes in the twisted Yang-Mills theory are actually trapped along $\Sigma$. Of course, once we identify which fermionic zero-modes are trapped along $\Sigma$, supersymmetry determines which bosonic zero-modes are trapped.

For the following analysis, we need only work in a small neighborhood of $\Sigma \subset S$, which we parametrize with local holomorphic coordinates $\left(s^{1}, s^{2}\right)$. Since $\varphi$ vanishes to first-order on $\Sigma$ by assumption, $\varphi$ takes the local form

$$
\varphi=t s^{2} d s^{1} \wedge d s^{2}
$$

According to (4.49), $\Sigma$ corresponds to the locus $s^{2}=0$. Hence $s^{1}$ is a coordinate along $\Sigma$, and $s^{2}$ is a coordinate on the normal bundle $N_{\Sigma / S}$ to $\Sigma$ inside $S$. Similarly, the conjugate $\bar{\varphi}$ is given by

$$
\bar{\varphi}=t \bar{s}^{2} d \bar{s}^{\overline{1}} \wedge d \bar{s}^{2}
$$

We now want to solve the equations of motion for the twisted fermions locally near $\Sigma$ in the background described by (4.49). According to Appendix C, the relevant terms in the twisted Yang-Mills action which determine the fermionic equations of 
motion on $S$ are

$$
\begin{aligned}
I_{S}= & \int_{\mathbb{R}^{3,1} \times S} d^{4} x \operatorname{Tr}\left(\chi^{\alpha} \wedge \bar{\partial}_{A} \psi_{\alpha}+\bar{\chi}_{\dot{\alpha}} \wedge \partial_{A} \bar{\psi}^{\dot{\alpha}}+2 i \sqrt{2} \omega \wedge \partial_{A} \eta^{\alpha} \wedge \psi_{\alpha}-2 i \sqrt{2} \omega \wedge \bar{\partial}_{A} \bar{\eta}_{\dot{\alpha}} \wedge \bar{\psi}^{\dot{\alpha}}\right. \\
& \left.-\frac{1}{2} \bar{\psi}_{\dot{\alpha}}\left[\bar{\varphi}, \bar{\psi}^{\dot{\alpha}}\right]+\frac{1}{2} \psi^{\alpha}\left[\varphi, \psi_{\alpha}\right]+\sqrt{2} \eta^{\alpha}\left[\bar{\varphi}, \chi_{\alpha}\right]+\sqrt{2} \bar{\eta}_{\dot{\alpha}}\left[\varphi, \bar{\chi}^{\dot{\alpha}}\right]\right)+\cdots
\end{aligned}
$$

Hence the fermionic wavefunctions near $\Sigma$ satisfy

$$
\begin{aligned}
\bar{\partial}_{A} \psi^{\alpha}-\sqrt{2}\left[\bar{\varphi}, \eta^{\alpha}\right] & =0, & \partial_{A} \bar{\psi}^{\dot{\alpha}}+\sqrt{2}\left[\varphi, \bar{\eta}^{\dot{\alpha}}\right] & =0, \\
\omega \wedge \bar{\partial}_{A} \bar{\eta}^{\dot{\alpha}}+\frac{i}{2 \sqrt{2}}\left[\bar{\varphi}, \bar{\psi}^{\dot{\alpha}}\right] & =0, & \omega \wedge \partial_{A} \eta^{\alpha}+\frac{i}{2 \sqrt{2}}\left[\varphi, \psi^{\alpha}\right] & =0,
\end{aligned}
$$

as well as

$$
\begin{aligned}
\omega \wedge \partial_{A} \psi^{\alpha}+\frac{i}{2}\left[\bar{\varphi}, \chi^{\alpha}\right] & =0, & \omega \wedge \bar{\partial}_{A} \bar{\psi}^{\dot{\alpha}}-\frac{i}{2}\left[\varphi, \bar{\chi}^{\dot{\alpha}}\right] & =0, \\
\bar{\partial}_{A} \chi^{\alpha}-\left[\varphi, \psi^{\alpha}\right] & =0, & \partial_{A} \bar{\chi}^{\dot{\alpha}}-\left[\bar{\varphi}, \bar{\psi}^{\dot{\alpha}}\right] & =0 .
\end{aligned}
$$

To look for fermion zero-modes trapped along $\Sigma$, we take all derivatives in (4.52) and (4.53) to act in the direction normal to $\Sigma$. We also assume that $\omega$ takes the canonical Euclidean form near $\Sigma$. As a result, in (4.52) only the components of $\psi^{\alpha}$ and $\bar{\psi}^{\dot{\alpha}}$ which are tangent to $\Sigma$ appear, and in (4.53) only the components of $\psi^{\alpha}$ and $\bar{\psi}^{\dot{\alpha}}$ which are normal to $\Sigma$ appear.

If $\Psi$ and $\widetilde{\Psi}$ represent any pair of fermions appearing in (4.52) and (4.53), then $\Psi$ and $\widetilde{\Psi}$ will have a zero-mode trapped along $\Sigma$ if these fermions satisfy classical equations of the schematic form (we ignore irrelevant constants)

$$
\begin{aligned}
& \frac{\partial \Psi}{\partial s^{2}}+\bar{s}^{2} \widetilde{\Psi}=0 \\
& \frac{\bar{\partial} \widetilde{\Psi}}{\bar{\partial} \bar{s}^{2}}+s^{2} \Psi=0
\end{aligned}
$$

implying that $\Psi$ and $\widetilde{\Psi}$ behave near $\Sigma$ as $\exp \left(-\left|s^{2}\right|^{2}\right)$. Given the local expressions for $\varphi$ and $\bar{\varphi}$ in (4.49) and (4.50), one can then check that the fermions in (4.52) 
do not have zero-modes localized along $\Sigma$. In contrast, each bifundamental fermion in (4.53) has precisely one such zero-mode, with Gaussian decay along the normal direction to $\Sigma$. In fact, since we are free to scale the Kähler metric near $\Sigma$ as we wish, the Gaussian decay away from $\Sigma$ can be made arbitrarily fast.

We thus obtain massless bifundamental matter localized along $\Sigma$ and associated to the following pairs of twisted fermions,

$$
\left(\begin{array}{c}
\psi_{\frac{\alpha}{2} d \bar{s}^{2}} \\
\chi_{12}^{\alpha} d s^{1} \wedge d s^{2}
\end{array}\right), \quad\left(\begin{array}{c}
\bar{\psi}_{2}^{\dot{\alpha}} d s^{2} \\
\bar{\chi} \frac{\dot{\alpha}}{12} d \bar{s}^{\overline{1}} \wedge d \bar{s}^{2}
\end{array}\right) .
$$

By appropriately raising or lowering indices using the Kähler metric on $S$, we naturally regard the fermion zero-modes derived from $\left(\psi_{\overline{2}}^{\alpha}, \bar{\chi}_{\overline{12}}^{\dot{\alpha}}\right) \equiv\left(\psi^{\alpha 2}, \bar{\chi}_{\overline{1}}^{\dot{\alpha} 2}\right)$ as transforming along $\Sigma$ in the bundles $N_{\Sigma / S}$ and $\bar{\Omega}_{\Sigma}^{1} \otimes N_{\Sigma / S}$, and similarly for the CPTconjugates in (4.55).

At first glance, this observation presents a small puzzle, since the corresponding fermions $\lambda_{\alpha}$ and $\bar{\lambda}_{\dot{\alpha}}^{c}$ in the defect theory transform as sections of $K_{\Sigma}^{1 / 2}$ and $\bar{K}_{\Sigma}^{1 / 2}$. However, we now recall that $\Sigma$ is itself defined by the vanishing of $\varphi_{0}$, a section of $K_{S}$. Thus the normal bundle $N_{\Sigma / S}$ is isomorphic to $\left.K_{S}\right|_{\Sigma}$,

$$
N_{\Sigma / S}=\left.K_{S}\right|_{\Sigma}
$$

We also recall that the adjunction formula implies

$$
K_{\Sigma}=\left.K_{S}\right|_{\Sigma} \otimes N_{\Sigma / S}
$$

Hence by (4.56) and (4.57),

$$
N_{\Sigma / S}=K_{\Sigma}^{1 / 2}
$$

As a result, the massless fermions localized along $\Sigma$ in the eight-dimensional YangMills theory on $S$ can be identified with the defect fermions $\lambda_{\alpha}$ and $\bar{\lambda}_{\dot{\alpha}}^{c}$ on $\Sigma$ which we initially introduced by hand.

Another important consequence of the isomorphism in (4.58) is that it establishes a canonical choice of spin structure on $\Sigma$. Namely, we use the spin structure associated to the normal bundle $N_{\Sigma / S}$ to define the defect fields on $\Sigma$. 


\subsection{Unfolding Singularities via Surface Operators}

As shown in Section 3, when the singularity type remains constant over all of $S$, the primitive Casimir invariants constructed from the vevs of $\varphi$ exactly match all possible ways that a singularity of general $A D E$ type can unfold. In this Section we demonstrate that the gauge theory degrees of freedom of the intersecting sevenbrane theory match to the possible unfoldings of a more general class of F-theory compactifications where the singularity type enhances along a real codimension two subspace of $S$ corresponding to a matter curve.

Given the precise match between gauge theory and geometry in the absence of a matter curve, it is natural to suspect that the primitive Casimir invariants of $\varphi$ still describe all possible ways to unfold the singularity type for more general F-theory compactifications. Indeed, as seen in Section 4.1, the possible deformations are locally identical to the case of the pure seven-brane theory. An immediate objection to this proposal follows from inspection of tables 4.19, 4.20 and 4.21 as well as the analogous results for the $A$ - and $D$ - type singularities. Indeed, the bundle assignments for the primitive Casimir invariants only match to those of the deformation parameters of the geometry up to tensoring by $\mathcal{O}_{S}(n \Sigma)$ for some $n>0$. In this Section we give an explanation for this apparent mismatch. The essential ingredient in this analysis is that a non-zero vev for a six-dimensional field localized along the matter curve $\Sigma$ can source a surface operator in the eight-dimensional partially twisted theory. In particular, as shown in equation (4.43) the bulk field $\varphi$ develops a pole along $\Sigma$ whose residue is given by the condensate of massless fields living on $\Sigma$ :

$$
\varphi=\frac{\left\langle\left\langle\sigma^{c}, \sigma\right\rangle\right\rangle_{\mathrm{ad}(P)} d s^{1} \wedge d s^{2}}{\alpha}+\cdots
$$

This will effectively shift the bundles which the casimirs of $\varphi$ belong to. Moreover, even though we will not be using it in this paper, it follows from equation (4.45) that generic values of $\sigma$ and $\sigma^{c}$ can also cause the bulk gauge field to develop singularities along $\Sigma$.

We interpret these singularities as a surface operator. As noted in [17], the reduction of equations (4.40) and (4.45) in the directions normal to $\Sigma$ in $S$ corresponds to Hitchin's equations in the presence of a source term. This is quite similar to the operative definition of surface operators given in [36]. While the topological twist 
of the four-dimensional gauge theory in [36] does not contain a $(2,0)$ form, an analogous singularity develops in the one-forms of that twisted theory. In the present context, a surface operator corresponds to a pole in $\varphi$ along the curve $(\alpha=0)$. Because the pole in $\varphi$ arises from an F-term supersymmetry condition, the path integral over arbitrary field configurations for $\varphi$ on $S$ automatically localizes onto those configurations of the form (4.59), so we obtain a disorder operator associated to $\Sigma \subset S$.

In the rest of this subsection we further elaborate on the connection between surface operators of the partially twisted theory and the unfolding of singularities in an F-theory compactification which includes intersecting seven-branes. As a warmup, we first show how surface operators in the partially twisted eight-dimensional theory coupled to a six-dimensional defect describe seven-brane recombination in both perturbatively and non-perturbatively realized compactifications. Next, we show that when the corresponding complex deformation exists, there is an exact match between meromorphic vevs of $\varphi$ with a simple pole structure and an arbitrary unfolding of all $A D E$ singularities other than $E_{8}$. In the $E_{8}$ case, the matter curve, where the $E_{8}$ singularity enhances to a higher singularity, will lead to exotic matter structure, which has not been analyzed. ${ }^{20}$ We thus will not have anything to say about the unfolding of the $E_{8}$ singularity because this requires further knowledge of the exotic physics living on the "matter curve".

\subsubsection{Brane Recombination}

In this Section we demonstrate that surface operators in both perturbative and nonperturbatively realized intersecting brane configurations correspond to brane recombination in the compactification. In the context of perturbatively realized intersecting D-brane configurations, it is well-known that vevs for bifundamental matter trigger brane recombination. For example, a four-dimensional gauge theory with gauge group $U(1) \times U(1)$ and two Higgs fields with opposite $U(1)$ charges will break to the diagonal $U(1)$ subgroup for appropriate Higgs vevs. In string theory this change in rank is interpreted as brane recombination. See figure 3 for a depiction of this process.

To interpret brane recombination in terms of surface operators, first recall that

\footnotetext{
${ }^{20} \mathrm{In}$ appendix $\mathrm{G}$ we will discuss some related questions.
} 


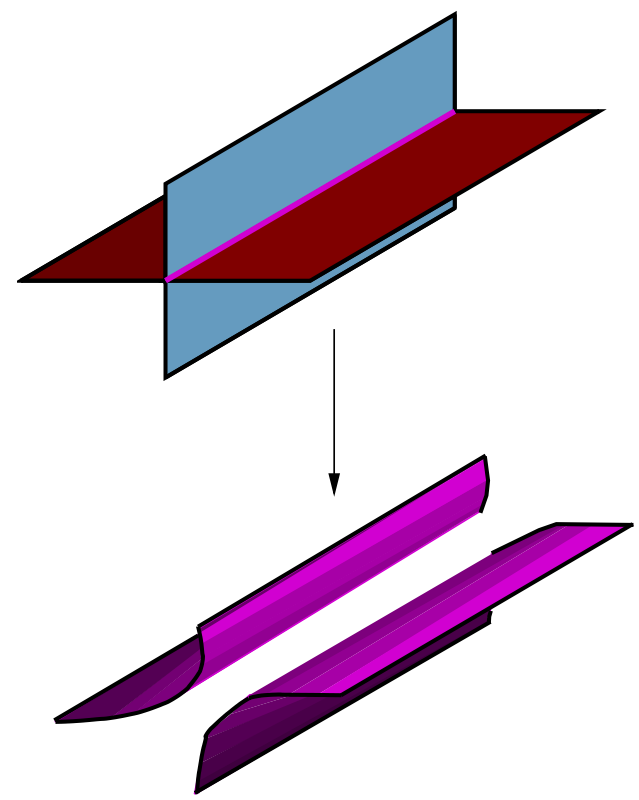

Figure 3: Prior to brane recombination, the common locus of two stacks of intersecting seven-branes lead to additional light degrees of freedom which propagate along a six-dimensional defect theory (top). When these light degrees of freedom condense, the branes recombine (bottom). 
the transverse intersection of $n+1$ D7-branes along $(z=0)$ with another stack of $m+1$ D7-branes along $(\alpha=0)$ is given by equation (4.6) with all $u_{l}$ and $t_{k}$ zero. For simplicity, we take the divisor $(\alpha=0)$ in the threefold base to be noncompact so that we may effectively treat the fields of this theory as non-dynamical constants. Returning to equation (4.40), note that the vev $\left\langle\left\langle\sigma^{c}, \sigma\right\rangle\right\rangle_{\operatorname{ad}(P)}$ corresponds to an $(n+1) \times(n+1)$ matrix of rank:

$$
l \leq \min (m+1, n+1)
$$

By a shift of coordinates, we may assume without loss of generality that the deformation in the $t_{i}$ is:

$$
\vec{t}=\left(\frac{b_{1}}{\alpha}+O\left(\alpha^{0}\right), \ldots, \frac{b_{l}}{\alpha}+O\left(\alpha^{0}\right), 0^{n+1-l}\right)
$$

so that the geometry now changes as:

$$
y^{2}=x^{2}+\alpha^{m+1} z^{n+1} \mapsto y^{2}=x^{2}+\alpha^{m+1} z^{n+1-l} \prod_{i=1}^{l}\left(z+\frac{b_{i}}{\alpha}\right)
$$

or,

$$
y^{2}=x^{2}+\alpha^{m+1-l} z^{n+1-l} \prod_{i=1}^{l}\left(\alpha z+b_{i}\right) .
$$

which corresponds to brane recombination of $l$ branes of the $(z=0)$ stack with $l$ branes of the $(\alpha=0)$ stack to form up to $l$ distinct stacks at $\left(\alpha z+b_{i}=0\right)$ for $i=1, \ldots, l$. Note that here $b_{i}$ is the $i^{\text {th }}$ eigenvalue of the matrix $\left\langle\left\langle\sigma^{c}, \sigma\right\rangle\right\rangle_{\operatorname{ad}(P)}$. Physically, the bound of (4.60) corresponds to the fact that once all of the branes of a stack have combined with other branes, there are none left over.

Starting from the local model:

$$
y^{2}=x^{2}+\alpha z^{n+1},
$$

a similar analysis establishes that the geometry:

$$
y^{2}=x^{2}+\alpha z^{n+1}+\sum_{i=2}^{n} \widehat{\alpha}_{i} z^{n-i}
$$


can be interpreted as a sequence of successive brane recombinations.

Because a similar analysis holds for brane recombination for $D$-type singularities, we now proceed to examples involving $E$-type branes. In fact, it is in principle possible that a similar analysis of $A$ - and $D$-type singularities will not be possible in the $E$-type case because this is an intrinsically non-perturbative feature of F-theory. Nevertheless, we now show that a sequential Higgsing of fields on the matter curve still produces a general deformation of lower singularity type.

In the next subsection we will present a more general analysis of unfolding of singularities in terms of surface operators, so for now we confine our remarks to some representative examples. The geometry:

$$
y^{2}=x^{3}+\alpha^{2} z^{4}
$$

corresponds to an $E_{6}$ singularity at $z=0$ and a non-compact $A_{2}$ singularity at $\alpha=0$. With conventions for deformations of $E$-type singularities as in $[28,22]$, breaking to $S O(10)$ will occur when the vev of $\varphi$ lies in the direction $(t,-2 t, t, t, t, t)$ of the Cartan subalgebra of $E_{6}$. After a suitable change of variables, the geometry is given by rescaling the result in [22] by factors of $\alpha$ :

$$
y^{2}=\alpha^{2} z^{4}-(4 \alpha z t-x)(2 \alpha z t+x)^{2}
$$

We note that when $t$ develops a first order pole along $\alpha$ with residue $b$, the geometry is now given by:

$$
y^{2}=\alpha^{2} z^{4}-(4 b z-x)(2 b z+x)^{2}
$$

so that the resulting matter in the $\mathbf{1 6}$ of $S O(10)$ is localized at $(b=0)$.

As a final example of brane recombination, the local geometry:

$$
y^{2}=x^{3}+\alpha x z^{3}
$$

corresponds to an $E_{7}$ singularity at $z=0$ and a non-compact $A_{1}$ singularity at $\alpha=0$. Breaking to $E_{6}$ will occur when the vev of $\varphi$ lies in the direction $(0,0,0,0,0, t, 0)$ of the Cartan subalgebra. Rescaling the result of [22] by powers of $\alpha$ yields:

$$
y^{2}=x^{3}+\alpha\left(x z^{3}+\alpha t^{2} z^{4}\right)
$$


where we have performed a shift in the $y$ and $z$ coordinates in order to cast the geometry in the above form. Note that when $t$ develops a first order pole along $\alpha=0$ with residue $b$, the resulting geometry is:

$$
y^{2}=x^{3}+\alpha x z^{3}+b^{2} z^{4}
$$

Proceeding step by step, a general geometry can be realized by further Higgsing the matter localized on Riemann surfaces.

\subsubsection{General Unfolding}

In the previous Section we presented a number of examples showing that general deformations of colliding singularities can be understood in terms of a sequence of brane recombinations induced by surface operators in the eight-dimensional partially twisted theory. In this Section we show that the general unfolding of a singularity again precisely matches to properties of the surface operator. To present this more general analysis, we shall assume throughout that the resulting deformation corresponds to a holomorphic section of an appropriate bundle on $S$. For example, when $S$ corresponds to a del Pezzo surface, $K_{S}$ is a strictly negative line bundle, so $K_{S}^{n}$ admits no holomorphic sections for $n>0$. Under these assumptions, we now match meromorphic vevs of $\varphi$ with a simple pole structure to an arbitrary unfolding of all $A D E$ singularities other than $E_{8}$.

We begin by describing the unfolding of a general $A_{n}$ singularity of the form:

$$
y^{2}=x^{2}+\alpha z^{n+1} .
$$

When the vev of $\varphi$ lies in a general direction of the Cartan subalgebra of $A_{n}$, the singularity deforms to equation (4.7) which we reproduce here:

$$
y^{2}=x^{2}+\alpha\left[z^{n+1}+s_{2} z^{n-1}+\cdots+s_{n+1}\right] .
$$

By definition of the $s_{i}$, when one of the $t_{i}$ 's develops a simple pole along $\alpha=0$, each $s_{i}$ will also contain a simple pole. In particular, this implies that each $\alpha \cdot s_{i}$ is a regular section which does not have to vanish at $\alpha=0$. Solving for a general deformation in terms of these regular sections, we see that the vevs of $\varphi$ indeed match to a general deformation of an $A_{n}$ singularity. 
Similarly, a general unfolding of the $D_{n}$ singularity:

$$
y^{2}=x^{2} z+\alpha^{2} z^{n-1}
$$

by the vev of $\varphi$ in a general direction of the Cartan subalgebra is the same as in [28] up to non-trivial powers of $\alpha$ which are required in order to preserve the overall homogeneity of the defining hypersurface equation:

$$
y^{2}=-x^{2} z+\alpha^{2} \frac{\prod_{i=1}^{n}\left(z+t_{i}^{2}\right)-\prod_{i=1}^{n} t_{i}^{2}}{z}+2 \alpha x \prod_{i=1}^{n} t_{i} .
$$

We note that when a single $t_{i}$ develops a pole along $\alpha=0$, the overall $\alpha$ dependence of all lower order deformations again cancels out. In this way, the vevs of $\varphi$ again match to the general class of deformations presented in equation (4.12).

While the above match between geometry and surface operators in the eightdimensional partially twisted theory is already non-trivial, we now show that this connection persists for the unfolding of $E_{6}$ and $E_{7}$ singularities. Consider first the unfolding of an $E_{6}$ singularity of the form:

$$
y^{2}=x^{3}+\alpha^{2} z^{4}
$$

by a vev of $\varphi$ in an arbitrary direction $\left(t_{1}, \ldots, t_{6}\right)$ of the Cartan subalgebra of $E_{6}$. The end result of this deformation is the same as in [28] up to scaling by powers of $\alpha$ :

$$
\begin{aligned}
y^{2} & =x^{3}+\alpha^{2} z^{4}+\varepsilon_{2}(2 \alpha)^{2} x z^{2}+\varepsilon_{5}(2 \alpha)^{3} x z+\varepsilon_{6}(2 \alpha)^{4} z^{2} \\
& +\varepsilon_{8}(2 \alpha)^{4} x+\varepsilon_{9}(2 \alpha)^{5} z+\varepsilon_{12}(2 \alpha)^{6}
\end{aligned}
$$

where the explicit functions $\varepsilon_{i}$ as functions of the $t_{i}$ are defined in Appendix 1 of [28] as functions of the elementary symmetric polynomials $s_{i}$ introduced earlier. The particular numerical coefficients have been chosen to conform with the conventions of [28].

Our expectation is that when the $\mathbf{2 7}$ localized along the matter curve in $S$ develops a vev, a pole in $\varphi$ will remove the overall $\alpha$ dependence of each type of deformation. In the present case, however, it follows from Appendix 1 of [28] that 
the leading order behavior of the $\varepsilon_{j}$ is:

$$
\varepsilon_{j} \propto\left(s_{1}\right)^{j}+O\left(t_{i}^{j-1}\right)
$$

where $O\left(t_{i}^{j-1}\right)$ denotes contributions of lower degree in each parameter $t_{i}$. In particular, when a single $t_{i}$ develops a pole along $\alpha=0, \varepsilon_{j}$ has a pole of order $j$ along $\alpha=0$. Returning to equation (4.77), it would at first appear that this branch does not match to a geometric unfolding of the singularity due to the presence of uncancelled poles in $\alpha$.

In fact, this is an artifact of the choice of coordinates used to present the most general possible unfolding. Returning to equation (4.77), we present the general deformation in terms of coordinates $X \equiv x$ and $Y \equiv y$ and:

$$
Z \equiv z-\frac{4}{27} \alpha\left(s_{1}\right)^{3}+\frac{1}{3} \alpha s_{1} s_{2}
$$

the definining hypersurface equation for a general deformation may now be written as:

$$
\begin{aligned}
Y^{2} & =X^{3}+\alpha^{2} Z^{4}+\beta_{2} \alpha^{2} X Z^{2}+\beta_{3} \alpha^{3} Z^{3}+\beta_{5} \alpha^{3} X Z \\
& +\beta_{6} \alpha^{4} Z^{2}+\beta_{8} \alpha^{4} X+\beta_{9} \alpha^{5} Z+\beta_{12} \alpha^{6}
\end{aligned}
$$

where the subscripts of the $\beta_{i}$ denote the degree in terms of polynomials in the $t_{i}$. Using the explicit expressions for the $\varepsilon_{i}$ given in Appendix 1 of [28], we find that the 
leading order behavior of the coefficients as polynomials in the $t_{i}$ is:

$$
\begin{aligned}
& \beta_{2} \alpha^{2}=\alpha^{2}\left(-\frac{4}{3}\left(s_{1}\right)^{2}+O\left(t_{i}\right)\right) \\
& \beta_{3} \alpha^{3}=\alpha^{3}\left(\frac{16}{27}\left(s_{1}\right)^{3}+O\left(t_{i}^{2}\right)\right) \\
& \beta_{5} \alpha^{3}=\alpha^{3}\left(-\frac{4}{3} s_{1}\left(s_{2}\right)^{2}+\frac{8}{3}\left(s_{1}\right)^{2} s_{3}+O\left(t_{i}^{2}\right)\right) \\
& \beta_{6} \alpha^{4}=\alpha^{4}\left(\frac{8}{9}\left(s_{1}\right)^{2}\left(s_{2}\right)^{2}-\frac{16}{9}\left(s_{1}\right)^{3} s_{3}+O\left(t_{i}^{3}\right)\right) \\
& \beta_{8} \alpha^{4}=\alpha^{4}\left(-\frac{1}{3}\left(s_{2}\right)^{4}+\frac{4}{3} s_{1}\left(s_{2}\right)^{2} s_{3}-\frac{4}{3}\left(s_{1}\right)^{2}\left(s_{3}\right)^{2}+O\left(t_{i}^{3}\right)\right) \\
& \beta_{9} \alpha^{5}=\alpha^{5}\left(\frac{4}{9} s_{1}\left(s_{2}\right)^{4}-\frac{16}{9}\left(s_{1}\right)^{2}\left(s_{2}\right)^{2} s_{3}+\frac{16}{9}\left(s_{1}\right)^{3}\left(s_{3}\right)^{2}+O\left(t_{i}^{4}\right)\right) \\
& \beta_{12} \alpha^{6}=\alpha^{6}\left(\frac{2}{27}\left(s_{2}\right)^{6}-\frac{4}{9} s_{1}\left(s_{2}\right)^{4} s_{3}+\frac{8}{9}\left(s_{1}\right)^{2}\left(s_{2}\right)^{2}\left(s_{3}\right)^{2}\right) .
\end{aligned}
$$

When a single $t_{i}$ develops a simple pole, each $s_{i}$ also develops a simple pole along $\alpha=0$. By inspection of the above result, we thus conclude that each product $\beta_{i} \alpha^{k}$ above is still regular and has no leading order dependence on $\alpha$. As for the $A$ - and $D$ type singularities, this implies that an arbitrary unfolding of the singularity matches to some choice of eigenvalues for $\varphi$. This is a highly non-trivial match between possible deformations of the singularity and the partially twisted seven-brane theory!

In a similar fashion, we now consider unfolding an $E_{7}$ singularity of the form:

$$
y^{2}=x^{3}+\alpha x z^{3}
$$

by a vev of $\varphi$ in an arbitrary direction $\left(t_{1}, \ldots, t_{7}\right)$ of the Cartan subalgebra of $E_{7}$. Up to powers of $\alpha$ introduced to preserve homogeneity, the unfolding of $E_{7}$ in [28] is:

$$
\begin{aligned}
y^{2} & =-x^{3}+16 \alpha x z^{3}+\varepsilon_{2} \alpha x^{2} z+\varepsilon_{6} \alpha^{2} x^{2}+\varepsilon_{8} \alpha^{3} x z \\
& +\varepsilon_{10} \alpha^{4} z^{2}+\varepsilon_{12} \alpha^{4} x+\varepsilon_{14} \alpha^{5} z+\varepsilon_{18} \alpha^{6}
\end{aligned}
$$

where the $\varepsilon_{i}$ as functions of the $t_{j}$ are defined in Appendix 2 of [28]. As before, the leading order behavior of $\varepsilon_{i} \propto\left(s_{1}\right)^{i}$ so that a simple pole in one of the $t$ 's would 
appear to not admit a geometric interpretion. As in the case of the $E_{6}$ singularity, we now show that this is an artifact of the choice of coordinates.

To this end, we first shift the $x$ coordinate via the substitution:

$$
x \mapsto x+\frac{1}{3}\left(\varepsilon_{2} \alpha z+\varepsilon_{6} \alpha^{2}\right) .
$$

Setting $Y \equiv y$ and $X \equiv x$, we also define:

$$
Z \equiv z-\frac{1}{16} \alpha\left(s_{1}\right)^{4}+\frac{1}{6} \alpha\left(s_{1}\right)^{2} s_{2}-\frac{1}{6} \alpha s_{1} s_{3}
$$

so that the defining hypersurface equation for a general deformation may now be written as:

$$
\begin{aligned}
Y^{2} & =-X^{3}+16 \alpha X Z^{3}+\beta_{2} \alpha^{2} Z^{4}+\beta_{4} \alpha^{2} X Z^{2}+\beta_{6} \alpha^{3} Z^{3} \\
& +\beta_{8} \alpha^{3} X Z+\beta_{10} \alpha^{4} Z^{2}+\beta_{12} \alpha^{4} X+\beta_{14} \alpha^{5} Z+\beta_{18} \alpha^{6}
\end{aligned}
$$

where as before, the subscripts indicate the degree of each $\beta_{i}$ in terms of polynomials in the $t_{i}$. The explicit leading order behavior of each $\beta_{i}$ as a polynomial in the $t_{i}$ may be found in Appendix F.

It follows from the results in Appendix $\mathrm{F}$ that a simple pole in one of the $t_{i}$ 's exactly cancels the overall $\alpha$ dependence of each deformation parameter. In particular, this implies that the degrees of freedom of the partially twisted $E_{7}$ theory with a defect exactly matches the unfolding of the singularity.

\subsection{Chiral Matter and Yukawa Couplings from $\Sigma$}

Having determined how the partially twisted seven-brane theory couples to a sixdimensional defect, we now determine some basic properties of supersymmetric vacua in the associated four-dimensional effective theory. Consider first the trivial case where the matter curve is given by a flat $T^{2}$. In this case, $\boldsymbol{\Lambda}$ and $\boldsymbol{\Lambda}^{c}$ correspond to two four-dimensional $\mathcal{N}=1$ chiral superfields transforming in complex conjugate representations. Indeed, these chiral multiplets determine an $\mathcal{N}=2$ hypermultiplet. In the special case where $U$ is a real representation of $G_{S}$, the six-dimensional fields can also organize into a half-hypermultiplet. This reduces to a single four-dimensional 
$\mathcal{N}=1$ chiral multiplet.

In the presence of potentially non-trivial background gauge field configurations on $S$ and $S^{\prime}$, the resulting zero mode spectrum is determined by bundle valued Dolbeault cohomology groups with support on $\Sigma$. The net chirality of the spectrum is then given by a topological invariant which is uniquely fixed by the representation and gauge bundle content of the six-dimensional fields localized on $\Sigma$. Because much of this discussion parallels a similar treatment given for the pure seven-brane theory, our discussion will be brief. As opposed to the case of the pure seven-brane theory wrapping a Hirzebruch or del Pezzo surface, we find that the non-trivial coupling between bulk gauge fields propagating on a compact surface and six-dimensional fields localized along $\Sigma$ induces non-trivial Yukawa couplings among the zero modes. We conclude this Section by presenting some toy models which further explicate these results.

\subsubsection{Massless Spectrum}

In this subsection we determine the massless particle spectrum in four dimensions of fields localized along a Riemann surface $\Sigma$ in $S$ in the presence of a potentially non-trivial background gauge field configuration. We begin with an analysis of the relevant group theory. Proceeding in a parallel fashion to the case of the pure seven-brane theory, non-trivial background gauge field configurations on $S$ and $S^{\prime}$ which take values in subgroups $H_{S} \subset G_{S}$ and $H_{S^{\prime}} \subset G_{S^{\prime}}$ will break $G_{S} \times G_{S^{\prime}}$ to the commutant subgroup. In many applications, $S^{\prime}$ is non-compact so that the associated gauge group factor is non-dynamical. As before, we let $\Gamma_{S}$ denote the maximal subgroup of $G_{S}$ such that $G_{S} \supset \Gamma_{S} \times H_{S}$, with similar notation for $\Gamma_{S^{\prime}}$. Letting $\Gamma=\Gamma_{S} \times \Gamma_{S^{\prime}}$ and $H=H_{S} \times H_{S^{\prime}}$, decomposing $U \times U^{\prime}$ into irreducible representations of $\Gamma \times H$ yields:

$$
U \otimes U^{\prime}=\bigoplus_{j}\left(\nu_{j}, V_{j}\right)
$$

A similar decomposition holds for the bundle $\mathcal{U} \otimes \mathcal{U}^{\prime}$. In the obvious notation, we let

$\mathcal{V}_{j}$ denote the corresponding bundle which transforms as a representation $V_{j}$ of $H$.

Because the supercurrent is covariantly constant in the partially twisted sixdimensional theory, it is enough to specify the massless spectrum of fermions. Taking 
into account the additional twist by an ambient line bundle on $\Sigma$ so that the resulting fermions transform as zero- and one-forms on $\Sigma$, it follows that the fermions $\lambda_{\alpha \nu_{j}}$ and $\lambda_{\alpha \nu_{j}^{*}}^{c}$ are both annihilated by $\bar{\partial}_{A+A^{\prime}}$ and $\bar{\partial}_{A+A^{\prime}}^{\dagger}$ and therefore also by the Laplacian $\Delta_{\bar{\partial}}=\bar{\partial}_{A+A^{\prime}} \bar{\partial}_{A+A^{\prime}}^{\dagger}+\bar{\partial}_{A+A^{\prime}}^{\dagger} \bar{\partial}_{A+A^{\prime}}$. The chiral spectrum is therefore:

$$
\begin{aligned}
& \lambda_{\alpha \nu_{j}} \in H_{\frac{0}{\partial}}\left(\Sigma, K_{\Sigma}^{1 / 2} \otimes \mathcal{V}_{j}\right) \\
& \lambda_{\alpha \nu_{j}^{*}}^{c} \in H_{\frac{0}{\partial}}\left(\Sigma, K_{\Sigma}^{1 / 2} \otimes \mathcal{V}_{j}^{*}\right) \simeq H_{\frac{1}{\partial}}\left(\Sigma, K_{\Sigma}^{1 / 2} \otimes \mathcal{V}_{j}\right)^{*}
\end{aligned}
$$

Using the analogue on $\Sigma$ of the isomorphism in (3.43), the anti-chiral spectrum is:

$$
\begin{aligned}
& \bar{\lambda}_{\dot{\alpha} \nu_{j}^{*}} \in \overline{H_{\frac{0}{\partial}}\left(\Sigma, K_{\Sigma}^{1 / 2} \otimes \mathcal{V}_{j}\right)} \simeq H_{\frac{0}{\partial}\left(\Sigma, K_{\Sigma}^{1 / 2} \otimes \mathcal{V}_{j}\right)^{*}} \\
& {\overline{\lambda^{c}}}_{\dot{\alpha} \nu_{j}} \in \overline{H_{\bar{\partial}}^{0}\left(\Sigma, K_{\Sigma}^{1 / 2} \otimes \mathcal{V}_{j}^{*}\right)} \simeq H_{\frac{1}{\partial}\left(\Sigma, K_{\Sigma}^{1 / 2} \otimes \mathcal{V}_{j}\right)}
\end{aligned}
$$

where in the above we have used Serre duality. Comparing equations (4.98) and (4.99) with (4.100) and (4.101), we observe that the resulting spectrum is manifestly CPT-invariant. It now follows that the net number of generations minus antigenerations transforming in the representation $\nu_{j}$ is:

$$
n_{\nu_{j}}-n_{\nu_{j}^{*}}=h^{0}\left(\Sigma, K_{\Sigma}^{1 / 2} \otimes \mathcal{V}_{j}\right)-h^{1}\left(\Sigma, K_{\Sigma}^{1 / 2} \otimes \mathcal{V}_{j}\right)=\chi\left(\Sigma, K_{\Sigma}^{1 / 2} \otimes \mathcal{V}_{j}\right)
$$

The Euler character $\chi\left(\Sigma, K_{\Sigma}^{1 / 2} \otimes \mathcal{V}_{j}\right)$ is now given by the analogue of the index theorem found in equation (3.49) for the Riemann surface $\Sigma$. Returning to equation (4.102), we therefore find:

$$
n_{\nu_{j}}-n_{\nu_{j}^{*}}=(1-g) \mathrm{rk}\left(K_{\Sigma}^{1 / 2} \otimes \mathcal{V}_{j}\right)+\int_{\Sigma} c_{1}\left(K_{\Sigma}^{1 / 2} \otimes \mathcal{V}_{j}\right)
$$

where $g$ denotes the genus of the Riemann surface $\Sigma$.

\subsubsection{More Yukawa Couplings}

In this Section we demonstrate that as opposed to the pure seven-brane theory, nontrivial Yukawa couplings can originate from the coupling of the bulk gauge fields to the matter curve. As explained near equation (4.32), it is convenient to treat $\boldsymbol{\Lambda}$ and 
$\boldsymbol{\Lambda}^{c}$ as a collection of four-dimensional fields labelled by points on $\Sigma$. Labelling all zero mode solutions of the bulk and defect theory by $\alpha, \beta$ and $\gamma$, the resulting cubic superpotential term now follows from equation (4.32):

$$
d_{\alpha \beta \gamma}=\int_{\Sigma} c_{i j k}\left(\boldsymbol{\Lambda}^{c, \alpha, i}\left(\mathbf{A}^{\beta, j}+\mathbf{A}^{\prime \beta^{\prime}, j}\right) \mathbf{\Lambda}^{\gamma, k}\right)
$$

where the indices $i, j, k$ are group indices in $G_{\Sigma} \supset G_{S} \times G_{S^{\prime}}$ and $c_{i j k}$ is a structure constant associated with the decomposition to $\Gamma_{S} \times \Gamma_{S^{\prime}}$.

Before closing this subsection, we now elaborate on the geometric content of the coupling between two fields localized on $\Sigma$ in representations $\nu_{1}$ and $\nu_{2}$ and a bulk field transforming in a representation $\tau$ with associated bundles $\mathcal{V}_{1}, \mathcal{V}_{2}$ and $\mathcal{T}$ which transform in representations of the structure group of the instanton configuration. A non-trivial Yukawa coupling corresponds to a tri-linear map:

$$
H \frac{0}{\partial}\left(\Sigma, K_{\Sigma}^{1 / 2} \otimes \mathcal{V}_{1}\right) \otimes H \frac{0}{\partial}\left(\Sigma, K_{\Sigma}^{1 / 2} \otimes \mathcal{V}_{2}\right) \otimes H \frac{1}{\partial}\left(\Sigma, \mathcal{T}_{\Sigma}\right) \rightarrow \mathbb{C}
$$

where $\mathcal{T}_{\Sigma}$ denotes the restriction of $\mathcal{T}$ to $\Sigma$. This map is natural in the sense that $H_{\frac{1}{\partial}}\left(\Sigma, \mathcal{T}_{\Sigma}\right)$ canonically pairs with $H_{\frac{0}{\partial}}^{0}\left(\Sigma, K_{\Sigma}^{1 / 2} \otimes \mathcal{V}_{1}\right) \otimes H_{\frac{0}{\partial}}^{0}\left(\Sigma, K_{\Sigma}^{1 / 2} \otimes \mathcal{V}_{2}\right)$ because of Serre duality on $\Sigma$ :

$$
H \frac{1}{\partial}\left(\Sigma, \mathcal{T}_{\Sigma}\right) \simeq H \frac{0}{\partial}\left(\Sigma, K_{\Sigma} \otimes \mathcal{T}_{\Sigma}^{*}\right)^{*}=H \frac{0}{\partial}\left(\Sigma, K_{\Sigma} \otimes \mathcal{V}_{1} \otimes \mathcal{V}_{2}\right)^{*}
$$

where in the final equality we have used the fact that the corresponding F-term only transforms as a gauge invariant singlet provided $\mathcal{T}_{\Sigma}=\mathcal{V}_{1}^{*} \otimes \mathcal{V}_{2}^{*}$. In this final form for $H \frac{1}{\partial}\left(\Sigma, \mathcal{I}_{\Sigma}\right)$, we see that the two fields on $\Sigma$ indeed pair naturally with the dual cohomology group which describes a component of the bulk gauge field.

\subsubsection{A Refined Toy Model}

Although we shall defer the construction of more realistic GUTs to future work in [19], we now explain how to generate a three generation $S O(10)$ toy model with non-trivial Yukawa couplings. We find it encouraging that many of the broadest features of $S O(10)$ GUTs can be achieved simply using supersymmetric gauge field configurations corresponding to line bundles on $S$. Consider a seven-brane wrapping a del Pezzo 3 surface with $G_{S}=S O(12)$ coupled to a defect. The charged matter 
localized along the defect is given by a six-dimensional half hypermultiplet in the 32 spinor representation. We assume that the matter curve is given by an exceptional curve $\Sigma$ with homology class $E_{1}$ in $H_{2}\left(d P_{3}, \mathbb{Z}\right)$. While it is important to describe the explicit geometry of the F-theory compactification, in order to focus on the most salient features of the toy model we shall defer such issues to future investigations.

The bulk gauge group $G_{S}=S O(12)$ breaks to $S O(10) \times U(1)$ in the presence of a supersymmetric gauge field configuration which takes non-trivial values in the $U(1)$ factor. This corresponds to a supersymmetric line bundle $\mathcal{L}$ on the surface $S$. The adjoint $\mathbf{6 6}$ and spinor $\mathbf{3 2}$ decompose under this subgroup as:

$$
\begin{aligned}
S O(12) & \supset S O(10) \times U(1) \\
\mathbf{6 6} & \rightarrow \mathbf{4 5}_{0}+\mathbf{1}_{0}+\mathbf{1 0}_{2}+\mathbf{1 0}_{-2} \\
\mathbf{3 2} & \rightarrow \mathbf{1 6}_{1}+\overline{\mathbf{1 6}}_{-1}
\end{aligned}
$$

so that the resulting matter content in four dimensions is:

$$
\begin{aligned}
\mathbf{1 0}_{2} & \in H \frac{1}{\partial}\left(S, \mathcal{L}^{2}\right) \\
\mathbf{1 0}_{-2} & \in H \frac{1}{\partial}\left(S, \mathcal{L}^{-2}\right) \\
\mathbf{1 6}_{1} & \in H \frac{0}{\partial}\left(\Sigma, K_{\Sigma}^{1 / 2} \otimes \mathcal{L}_{\Sigma}\right) \\
\overline{\mathbf{1 6}}_{-1} & \in H \frac{0}{\partial}\left(\Sigma, K_{\Sigma}^{1 / 2} \otimes \mathcal{L}_{\Sigma}^{-1}\right),
\end{aligned}
$$

where $\mathcal{L}_{\Sigma}$ denotes the restriction of $L$ to the matter curve $\Sigma$.

In Appendix E we show that there exist a family of Kähler classes such that the line bundle:

$$
\mathcal{L}=\mathcal{O}_{S}\left(a_{1} E_{1}+a_{2} E_{2}+a_{3} E_{3}\right)
$$

is supersymmetric provided $a_{i} a_{j}<0$ for some $i \neq j$.

To achieve three 16's with a minimal number of net 10's in the bulk, we take $a_{1}=-3, a_{2}=2, a_{3}=2$ so that:

$$
\begin{aligned}
\mathcal{L} & =\mathcal{O}_{S}\left(-3 E_{1}+2 E_{2}+2 E_{3}\right) \\
\mathcal{L}_{\Sigma} & =\mathcal{O}_{\Sigma}(+3)
\end{aligned}
$$

Because they are bulk fields, the net number of massless chiral fields transforming in 
the $\mathbf{1 0}$ follows from equations (3.50) and (3.52):

$$
\begin{aligned}
n_{\mathbf{1 0}_{2}} & =32 \\
n_{\mathbf{1 0}_{-2}} & =34 \\
n_{\mathbf{1 0}_{-2}}-n_{\mathbf{1 0}_{2}} & =2 .
\end{aligned}
$$

Next consider the six-dimensional half hypermultiplet localized along the matter curve. The zero modes for the $\mathbf{1 6}$ 's and $\overline{\mathbf{1 6}}$ 's are respectively given by harmonic representatives in the cohomology groups (4.112) and (4.113). The total number transforming in each representation is:

$$
\begin{aligned}
& n_{\mathbf{1 6}}=\operatorname{dim} H_{\bar{\partial}}^{0}\left(\Sigma, K_{\Sigma}^{1 / 2} \otimes \mathcal{L}_{\Sigma}\right)=\operatorname{dim} H \frac{0}{\partial}\left(\Sigma, \mathcal{O}_{\Sigma}(2)\right)=3 \\
& n_{\overline{\mathbf{1 6}}}=\operatorname{dim} H_{\bar{\partial}}\left(\Sigma, K_{\Sigma}^{1 / 2} \otimes \mathcal{L}_{\Sigma}^{-1}\right)=\operatorname{dim} H_{\bar{\partial}}^{0}\left(\Sigma, \mathcal{O}_{\Sigma}(-4)\right)=0
\end{aligned}
$$

Labelling the three zero modes on $\Sigma$ as $\mathbf{1 6}^{(i)}$ for $i=1,2,3$, it follows from equation (4.104) that the contribution to the superpotential from the bulk to surface interaction term is schematically of the form:

$$
W \supset \lambda_{i j} 16^{(i)} \times 16^{(j)} \times 10^{(S)}
$$

where $\mathbf{1 0}^{(S)}$ is shorthand for all possible contributions from the large number of zero mode solutions transforming in this representation of the $S O(10)$ GUT. Note that in this particular case, the structure constants of $G_{S}$ only allow the $\mathbf{1 0}_{-2}$ 's to couple to the 16's because of the $U(1)$ charges of all fields in the decomposition of $G_{S}$.

While we defer a full discussion of Yukawa couplings to [19], we now briefly comment on some general features of the flavor structure associated with coupling two matter fields on $\Sigma$ to a bulk field in $S$ for models in which such interaction terms are the sole contribution to the four-dimensional effective superpotential. While in the example just presented we have localized all three generations on a single matter curve, it is in principle possible to localize different generations on distinct matter curves, or to allow some generations to descend from bulk fields. This can in principle be used to induce non-trivial texture zeroes in the Yukawa matrices. As an example, suppose we have three 16's localized on three distinct matter curves in $S$ with some number of bulk $\mathbf{1 0}^{(S)}$ 's. Labelling the three generations as $\mathbf{1 6}^{(i)}$ for $i=1,2,3$, the Yukawa couplings are still described by equation (4.122) where now the Yukawa 
matrix takes the schematic form:

$$
\lambda_{i j} \sim\left(\begin{array}{ccc}
\lambda_{11} & 0 & 0 \\
0 & \lambda_{22} & 0 \\
0 & 0 & \lambda_{33}
\end{array}\right)
$$

As another example, suppose that we have constructed a consistent three generation $S O(10)$ GUT model with one 16 and a 10 coming from bulk zero modes on $S$ with two generations localized along a single matter curve. Denoting the bulk $\mathbf{1 6}$ by $\mathbf{1 6}^{(1)}$, we let $\mathbf{1 6}^{(2)}$ and $\mathbf{1 6}^{(3)}$ denote the two generations localized on the matter curve. In this case, the analogue of equation (4.122) implies that the Yukawa matrix takes the form:

$$
\lambda_{i j} \sim\left(\begin{array}{ccc}
0 & 0 & 0 \\
0 & \lambda_{22} & \lambda_{23} \\
0 & \lambda_{23} & \lambda_{33}
\end{array}\right) .
$$

\section{Multiple Intersections and More Yukawa Cou- plings}

While the generic singularity type of the elliptic Calabi-Yau fourfold corresponds to $G_{S}$ over $S$ with corresponding gauge group, simple genericity arguments imply that there exist subloci of complex codimension one and two in $S$ along which the rank of $G_{S}$ increases by one and two, respectively. This follows from the dictionary established between the unfolding of the singularity of the geometry and its interpretation in terms of fields of the partially twisted theory. For example, consider the case where $G_{S}=E_{6}$. In this case, the local geometry is:

$$
y^{2}=x^{3}+\alpha^{2} z^{4}
$$

where $\alpha$ is a section of a suitable bundle over $S$. When this bundle is non-trivial, there is a codimension one locus on $S$ where $\alpha=0$. Although the singularity type appears to degenerate when $\alpha=0$, there are higher order terms in this defining equation which do not correspond to light degrees of freedom for $\alpha \neq 0$. Along this 
locus, however, the next relevant term in the singularity is:

$$
y^{2}=x^{3}+\alpha^{2} z^{4}+\beta x z^{3}
$$

so that for generic points on the curve $\alpha=0$ the geometry contains an $E_{7}$ singularity. Note that in the case where $S$ is a del Pezzo surface, $K_{S}^{-1}$ is an ample line bundle so that when $\alpha$ has a section, $\beta$ does as well. In particular, this implies that for generic points of $S, \beta \neq 0$. The singularity type can enhance to even higher type along the discrete collection of points in $S$ where $\alpha=\beta=0$. Adding in the next highest term, the model is therefore of the form:

$$
y^{2}=x^{3}+\alpha^{2} z^{4}+\beta x z^{3}+\gamma z^{5}
$$

so that when $\alpha=\beta=0$ the geometry contains an $E_{8}$ type singularity.

This example is illustrative of the generic situation. Letting $r$ denote the rank of $G_{S}$, along curves $\Sigma_{j}$ the singularity type $G_{\Sigma_{j}}$ enhances to rank $r+1$. Moreover, at $l$ points $p_{I}^{(k)}$, some collection of curves $\Sigma_{i_{1}}, \ldots$, and $\Sigma_{i_{n}}$ intersect where $k=1, \ldots, l$ and $I$ denotes a mult-index in the $n$ variables. Along such points, the singularity type $G_{p_{I}^{(k)}}$ has rank $r+2$. While arguments based on dimension counting would suggest that only two matter curves can intersect, we shall argue that for exceptional type singularities, three curves can also generically meet. The above analysis implies that the singularity types obey the containment relations:

$$
G_{p_{I}^{(k)}} \supset G_{\Sigma_{j}} \times U(1) \supset G_{S} \times U(1) \times U(1) .
$$

See figure 4 for a depiction of the enhancement in singularity type along defects of the bulk theory on $S$.

As discussed in Section 4, one potential source of four-dimensional chiral matter localized along a curve $\Sigma_{j}$ originates from the local enhancement of singularity type from $G_{S}$ to $G_{\Sigma_{j}}$. In this Section we perform a similar analysis to analyze the last stage of enhancement to a singularity of type $G_{p_{I}^{(k)}}$.

Before proceeding to a more detailed analysis of rank two enhancement in singularity type, note that when $G_{S}=E_{7}$, the resulting $G_{p_{i j}^{(k)}}$ would appear to have rank nine. Because the largest compact exceptional group has rank eight, we conclude that the resulting physics is likely to be somewhat more exotic. This special case 
has been discussed in [23] and we defer further discussion to Appendix G. For now, we will assume that all of the singularities encountered are of $A D E$ type.

To better understand the physics associated with points $p_{I}$ where the singularity type enhances twofold, we first treat the case where $G_{p_{I}}$ is an $A$-type singularity. In this case, a perturbative treatment of the geometry in terms of intersecting D7-branes is available, and it is well-known that such configurations signal the presence of additional superpotential terms in the four-dimensional effective theory. Re-interpreting these results in terms of the general philosophy outlined in [22] yields a similar result for more general geometries which contain points of twofold enhancement in the singularity type. We find that multiple intersections of matter curves are generic for certain F-theory compactifications and moreover induce additional Yukawa couplings in the four-dimensional effective theory. To better illustrate these facts, we present explicit examples of this phenomenon for E-type singularities.

\subsection{Enhancement to $A$-Type Singularities}

As a representative example, we first consider the triple intersection of D7-branes wrapping three distinct divisors in the threefold base described by the local geometry:

$$
y^{2}=x^{2}+\left(z-t_{1}\right)^{n_{1}}\left(z-t_{2}\right)^{n_{2}}\left(z-t_{3}\right)^{n_{3}}
$$

so that there are six-dimensional bifundamentals of $U\left(n_{i}\right) \times U\left(n_{j}\right)$ localized at each pairwise intersection of divisors. ${ }^{21}$ When the $t_{i}$ are all regular sections, the three stacks of D7-branes wrap compact divisors in the threefold base. On the other hand, as explained in previous Sections, we can also consider the case where some of the $t_{i}$ are meromorphic sections so that some of these divisors are non-compact. Decomposing into four-dimensional $\mathcal{N}=1$ superfields $\Lambda$ and $\boldsymbol{\Lambda}^{c}$, by abuse of notation we shall often denote possible contributions from either type of superfield as $\boldsymbol{\Lambda}_{i \bar{j}}$ when the context is clear.

To proceed further, note that this situation is well-described by perturbative type IIB superstrings. In this context, we recall that a pair of intersecting D7-branes gives bifundamental fields $\Lambda_{i \bar{j}}$ on the intersection. Further, while triple intersections of

\footnotetext{
${ }^{21}$ In order to emphasize the connection with D-branes, in this Section we include the explicit $U(1)$ factor of the worldvolume gauge group so that the full gauge group is $U(n)$.
} 
a)

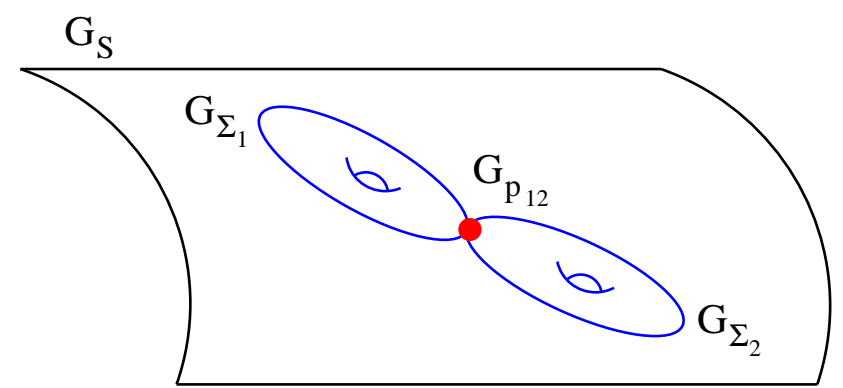

b)

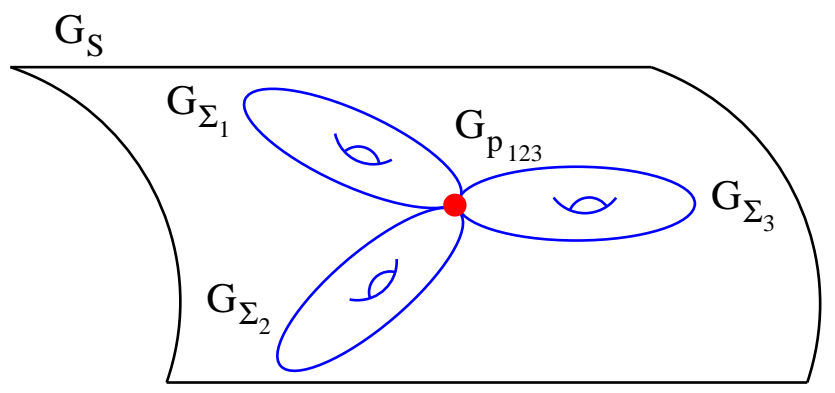

Figure 4: Depiction of the bulk eight-dimensional gauge theory defined by F-theory with singularity type $G_{S}$ at generic points of the complex surface $S$. Along a codimension one defect, the singularity type can generically enhance to the higher rank singularity $G_{\Sigma_{j}}$. At codimension two defects where distinct matter curves intersect, the geometry can enhance to the even higher rank singularity $G_{p_{i j}}$ when two curves intersect (a) and $G_{p_{i j k}}$ when three curves intersect (b). Contrary to expectations based on dimension counting, we find that case (b) is generic for geometries with exceptional type singularities. 
D7-branes at a point do not contribute additional matter fields, the disc diagram amplitude ending on the three D7-branes generates a superpotential term in the four dimensional effective theory between the $\boldsymbol{\Lambda}_{i \bar{j}}$. In fact, the superpotential computation localizes to constant maps of the disc to the point of the triple intersection. The end result is the superpotential term localized at the triple intersection point:

$$
\left.W \supset \boldsymbol{\Lambda}_{12} \boldsymbol{\Lambda}_{2 \overline{3}} \boldsymbol{\Lambda}_{3 \overline{1}}\right|_{p}
$$

We will now try to recover this well-known result in the framework of geometric singularities so that we can apply this methodology to more general examples and in particular to E-type singularities. Specializing to the case where $n_{1}=n_{2}=1$ and setting $n_{3}=n$ yields:

$$
y^{2}=x^{2}+z^{n}\left(z^{2}-\left(t_{1}+t_{2}\right) z+t_{1} t_{2}\right)
$$

where in passing from equation (5.5) to equation (5.7) we have set $t_{3}=0$ for notational simplicity. At generic points of $S$, the singularity type of the geometry at $z=0$ is an $A_{n-1}$ singularity. In this case, each $\Sigma_{i}$ corresponds to the locus $(z=0) \cap\left(z=t_{i}\right)$. Along the vanishing locus for each $\Sigma_{i}$, this appears to enhance to $A_{n}$ and at the discrete collection of points in $S$ where $t_{1}=t_{2}=0$, the singularity type enhances to $A_{n+1}$.

We now interpret this system from the perspective of the gauge theory. Recall that the $t_{i}$ denote non-zero vevs for the eigenvalues of the $\varphi$ form on $S$. Along the locus where only one $t_{i}$ vanishes, the generic $U(n) \times U(1)_{1} \times U(1)_{2}$ gauge group enhances to $U(n+1)_{i} \times U(1)_{i}$, where the subscript indicates the embedding inside $U(n+2)$. Indeed, given the partially broken gauge group $U(n) \times U(1)_{1} \times U(1)_{2}$, there are two ways in which such an embedding can occur. We note that there is one additional enhancement when the vevs $\varphi$ are proportional to the identity in the $U(2)$ direction so that $t_{1}=t_{2}$. A six-dimensional field in the bifundamental of two of the unitary group factors localizes along the defining equations for the corresponding $t^{\prime}$ 's. This additional field lies on another Riemann surface $\Sigma^{\prime}$ which is not localized in $S$. We note that this is consistent with the fact that the corresponding matter fields on $\Sigma^{\prime}$ are not charged under the gauge group $G_{S}$. Decomposing the adjoint representation of $U(n+2)$ to $U(n) \times U(1)_{1} \times U(1)_{2}$, we therefore conclude that there is a six-dimensional field in the $(\mathbf{n}, 0,+1)$ localized along the matter curve 
$(z=0) \cap\left(z=t_{1}\right)$, another six-dimensional field in the $(\overline{\mathbf{n}},-1,0)$ localized along $(z=0) \cap\left(z=t_{2}\right)$, and a third in the $(\cdot,+1,-1)$ localized along $\left(z=t_{1}\right) \cap\left(z=t_{2}\right)$.

At the common intersection point $t_{1}=t_{2}=0$, the wave functions of the three matter fields overlap. In fact, we can directly generalize the philosophy in [22] to the case at hand and study the theory from the viewpoint of the gauge theory $G_{p}=U(n+2)$. Viewing $t_{1}$ and $t_{2}$ as scalar fields in the Cartan of $U(2) \subset U(n+2)$, note that in the limit where the $t_{i}$ vanish, we have an eight-dimensional theory with gauge group $U(n+2)$. Turning on the $t_{i}$ 's has the effect of creating fields localized on each matter curve. Note that the eight-dimensional theory with gauge group $U(n+2)$ also contains a cubic Yukawa coupling between fields localized on matter curves. Evaluating the triple overlap of the three wavefunctions at the common point of intersection, we find a non-zero contribution to the superpotential in the four dimensional effective theory. We have thus recovered the perturbatively generated superpotential from those locations in the geometry where a twofold enhancement in the singularity type occurs.

Note that when $t_{1}$ and $t_{2}$ share a mutual pole, the wave function for the bifundamental of $U(1)_{1} \times U(1)_{2}$ along the corresponding Riemann surface can have non-compact support. In this case, this field appears as a coupling constant in the compact theory realized on the surface $S$. In the four dimensional effective theory, a non-zero vev for this bifundamental generates a mass term for the vector-like pair of fields in the fundamental and anti-fundamental of $U(n)$.

In the above analysis for $A$-type singularities, we have implicitly assumed that the $t_{i}$ are global sections. In general, this may not hold because only $t_{1}+t_{2}$ and $t_{1} t_{2}$ appear in the local presentation of the geometry. This more general case corresponds to geometries where the $t_{i}$ do not remain invariant under a monodromy in the fiber direction so that the singularity is not of 'split' type. As explained in [21], when the singularity is not of split type, the gauge group is reduced by some outer automorphism which in the perturbative string setup would correspond to orientifolding an $A_{n}$ singularity. Because our primary interest in this paper is GUT groups which descend from an $E$ type singularity, we shall not treat in any detail the action of outer automorphisms on the corresponding GUT groups.

A similar analysis holds when the highest singularity type of the geometry is $D_{n}$. In this case, the six-dimensional fields localized on matter curves are bifundamentals of $S O(2 n) \times U(m), S O(2 n) \times U\left(m^{\prime}\right)$ and $U(m) \times U\left(m^{\prime}\right)$. As in the case of $A$-type 
singularities, a cubic coupling corresponds to a mass term because the last type of bifundamental is non-dynamical in the four-dimensional effective theory.

\subsection{Cubic Couplings from Codimension Two}

Given the above example, we now describe how cubic couplings originate for more general geometries. As discussed above, we have a sequence of gauge groups

$$
G_{p_{I}} \supset G_{\Sigma_{j}} \times U(1) \supset G_{S} \times U(1) \times U(1)
$$

For each $\Sigma_{j}$ there exists a six-dimensional matter field $\boldsymbol{\Lambda}_{j}$ which can potentially lead to chiral matter in four dimensions. Each $\boldsymbol{\Lambda}_{j}$ is part of the adjoint representation of the bigger group $G_{p_{I}}$. The interaction term in the theory with gauge group $G_{p_{I}}$ induces an interaction between the fields $\Lambda_{j}^{a}$, where $a$ denotes a group theory index for $G_{p_{I}}$. Letting $f_{a b c}$ denote the structure constants of $G_{p_{I}}$, the resulting induced interaction term coming from the adjoint interaction for the seven-branes with gauge group $G_{p_{I}}$ is:

$$
f_{a b c} \Lambda_{j}^{a} \Lambda_{k}^{b} \Lambda_{l}^{c}
$$

We now interpret the geometric content of the above equation. Recall from the twisting of the six-dimensional defect theory that each $\boldsymbol{\Lambda}_{i}$ transforms as a section of the bundle $K_{\Sigma_{i}}^{1 / 2}$. On the other hand, we will now argue that there is a canonical identification:

$$
\left.K_{\Sigma_{1}} \otimes K_{\Sigma_{2}} \otimes K_{\Sigma_{3}}\right|_{p}=1
$$

Further, a mild generalization of the discussion in subsection 4.2.3 near equation (4.58) demonstrates that there is in fact a canonical choice of square root for each $K_{\Sigma}$ in equation (5.10). This uniquely fixes the overall sign of $\mathbf{1}^{1 / 2}$. To see equation (5.10), note that equation (4.58) implies:

$$
K_{\Sigma_{3}}=N_{\Sigma_{3} / S}^{2}
$$

and so we can write:

$$
\left.K_{\Sigma_{1}} \otimes K_{\Sigma_{2}} \otimes K_{\Sigma_{3}}\right|_{p}=\left.\left.\left(K_{\Sigma_{1}} \otimes N_{\Sigma_{3} / S}\right)\right|_{p} \otimes\left(K_{\Sigma_{2}} \otimes N_{\Sigma_{3} / S}\right)\right|_{p}=\mathbf{1}
$$


where the last equality follows from the fact that the $\Sigma_{i}$ 's intersect pairwise inside $S$.

We now describe the form of the resulting superpotential term in four dimensions. Letting $\boldsymbol{\Lambda}^{\alpha_{j}, a}$ denote one of the zero modes of $\boldsymbol{\Lambda}_{j}$ on $\Sigma_{j}, \alpha_{j}$ denotes an index which runs over the set of chiral zero modes and as before, $a$ denotes a group index. The resulting cubic superpotential term between the corresponding chiral fields is therefore given by:

$$
d_{\alpha_{j}, \beta_{k}, \gamma_{l}}=f_{a b c} \boldsymbol{\Lambda}_{j}^{\alpha_{j}, a}\left(p_{j k l}\right) \boldsymbol{\Lambda}_{k}^{\beta_{k}, b}\left(p_{j k l}\right) \boldsymbol{\Lambda}_{l}^{\gamma_{l}, c}\left(p_{j k l}\right)
$$

with similar conventions for $\beta_{k}$ and $\gamma_{l}$. We now proceed to some examples of the relevant group theory in the context of $E$-type singularities.

\subsection{Local Cubic Couplings for E-Type Singularities}

To illustrate the above formalism, we now show how cubic couplings can originate from $E$-type singularities. First consider the local geometry mentioned previously where $G_{p}=E_{8}, G_{\Sigma}=E_{7}$ and $G_{S}=E_{6}$ so that:

$$
y^{2}=x^{3}+\gamma z^{5}+\beta x z^{3}+\alpha^{2} z^{4} .
$$

Because we may locally trivialize $\gamma$ near the common vanishing locus of $\alpha$ and $\beta$, we may analyze the matter content of this theory as an $E_{8}$ gauge theory where $\varphi$ has developed a vev. The deformation in the Cartan which breaks $E_{8}$ to $E_{6} \times U(1) \times U(1)$ corresponds to the t-direction $\vec{t}=\left(t_{1}, t_{2}, 0,0,0,0,0,0\right)$. Decomposing the adjoint representation of $E_{8}$ under $E_{6} \times U(1) \times U(1)$, there are three 27's with $U(1)$ charges $(+1,0),(0,-1)$ and $(-1,+1)$ localized along the matter curves $\left(t_{1}=0\right),\left(t_{2}=0\right)$ and $\left(t_{1}=-t_{2}\right)$. Note that along each matter curve, the singularity type enhances to $E_{7}$. As discussed in Section 4, the matter curves can be read off from the geometry by writing $\alpha, \beta, \gamma$ as explicit functions of the $t_{i}$. It now follows that there are three matter curves in $S$ with the background $U(1)$ charge for each determining the total number of 27's on each curve. The overlap of the three wavefunctions at the common intersection points $t_{1}=t_{2}=0$ contributes an additional $27^{3}$ gauge invariant term to the superpotential. A similar analysis holds for more general breaking patterns. As another example, when $G_{p}=E_{7}, G_{\Sigma}=E_{6}$ and $G_{S}=S O(10)$, the resulting 
superpotential will contain the product $16 \times 16 \times 10$. This type of interaction term can originate from the unfolding:

$$
y^{2}=x^{3}+\gamma x z^{3}+\beta z^{4}+\alpha x^{2} z
$$

We wish to emphasize that even if the bulk gauge group is of classical $A$ - or $D$ type, a twofold enhancement in rank to an E-type singularity can generate Yukawa couplings which vanish identically in string perturbation theory. To illustrate this point, we note that while it is certainly possible to engineer a D-brane construction of an $S U(5)$ GUT model with three generations of $\overline{5}$ 's and 10's as in for example [5], a semi-realistic $S U(5)$ GUT must contain contributions to the superpotential of the form:

$$
W_{S U(5)} \supset \overline{\mathbf{5}}_{H} \times \overline{\mathbf{5}}_{M} \times \mathbf{1 0} \mathbf{0}_{M}
$$

and:

$$
W_{S U(5)} \supset 5_{H} \times 10_{M} \times 10_{M}
$$

where the subscripts $H$ and $M$ respectively denote Higgs and matter fields of the $S U(5)$ GUT. In perturbative type II string theory, the coupling of (5.17) is zero because the group indices only contract in the presence of a five index $\varepsilon$ tensor so that the purported coupling would violate the $U(1)$ charge associated with the $U(5)$ gauge symmetry of the D-brane. Note that even if this $U(1)$ lifts via a Green-Schwarz mechanism, in perturbative string theory it will persist as a global symmetry which can, however, be violated by non-perturbative effects.

From the perspective of geometry, we can expect to get 5's and $\overline{\mathbf{5}}$ 's from enhancement to an $A_{5}$ singularity and 10's from enhancement to a $D_{5}$ singularity. Whereas the resulting twofold enhancement to a $D$-type singularity can always be realized in perturbative D-brane constructions, for $S U(5)$ there is another possibility where the singularity type enhances to $E_{6}$. See figure 5 for a depiction of this enhancement. In such cases, the $78^{3}$ interaction of the $E_{6}$ gauge theory will contain a contribution of the form given by (5.17). Note that at other points of the geometry we can generically expect rank two enhancement to a $D$-type singularity. These points will lead to superpotential contributions of the form given by (5.16). This example illustrates our general philosophy that even for classical GUT groups, the presence of an E-type singularity in the geometry is important for phenomenology. 
i)

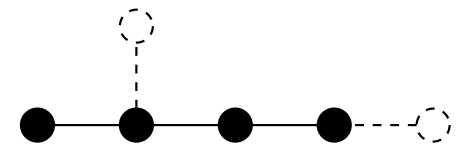

ii)

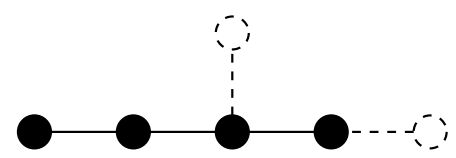

Figure 5: Dynkin diagrams which illustrate how the gauge group $S U(5)$ with corresponding singularity $A_{4}$ can enhance along matter curves to $A_{5}$ and $D_{5}$ and can undergo a further rank two enhancement at isolated points to either $D_{6}$ (i) or $E_{6}$ (ii). Whereas the first possibility can generically be realized in perturbative type II string theory constructions for any rank, the second case is exceptional and can give rise to Yukawa couplings which vanish perturbatively.

\section{A Final Toy Model}

In this Section we present a final toy model for an $S O(10)$ GUT which combines many of the ingredients described in the previous Sections. To this end, we treat the theory of a seven-brane wrapping a del Pezzo 3 surface with bulk gauge group $G_{S}=S O(12)$. In order to introduce chiral matter with non-trivial interactions, we analyze a model in which an enhancement by one rank occurs along some matter curves to both $E_{7}$ and $D_{7}$ type singularities. To allow a more varied class of interaction terms, we also require that these matter curves intersect at points such that the singularity type enhances to $E_{8}$. After introducing a supersymmetric gauge field configuration to induce a chiral matter spectrum in four dimensions, we determine the cubic coupling contributions to the superpotential in the four-dimensional effective theory.

We begin with an analysis of how deformations in the Cartan of the $E_{8}$ singularity localized at some point $p$ descend to a rank 7 singularity along matter curves and the rank 6 bulk gauge group $S O(12)$. The breaking pattern:

$$
E_{8} \supset S O(12) \times U(1)_{1} \times U(1)_{2}
$$

is achieved by noting that $S O(16)$ is a maximal subalgebra of $E_{8}$. Indeed, the adjoint representation of $E_{8}$ decomposes to the adjoint and spinor representations of 
$S O(16)$ as:

$$
\begin{aligned}
E_{8} & \supset S O(16) \\
\mathbf{2 4 8} & \rightarrow \mathbf{1 2 0}+\mathbf{1 2 8 .} .
\end{aligned}
$$

Because the adjoint of $E_{8}$ decomposes to $S O(12)$ via:

$$
\begin{aligned}
E_{8} & \supset S O(12) \times S U(2)_{1} \times S U(2)_{2} \\
\mathbf{2 4 8} & \rightarrow(\mathbf{6 6}, \mathbf{1}, \mathbf{1})+(\mathbf{1}, \mathbf{3}, \mathbf{1})+(\mathbf{1}, \mathbf{1}, \mathbf{3})+\left(\mathbf{3 2}^{\prime}, \mathbf{2}, \mathbf{1}\right)+(\mathbf{3 2}, \mathbf{1}, \mathbf{2})+(\mathbf{1 2}, \mathbf{2}, \mathbf{2})
\end{aligned}
$$

we can identify four matter curves in $S$ by examining the $U(1)$ charges of the fields. The half hypermultiplet content of each curve $\Sigma_{i}$ for $i=1, \ldots, 4$ is therefore given by a $32^{\prime}$ localized along $\Sigma_{1}$, a 32 along $\Sigma_{2}$, a 12 along $\Sigma_{3}$ and another 12 along $\Sigma_{4}$. Because the matter content from $\Sigma_{3}$ and $\Sigma_{4}$ is essentially identical, for illustrative purposes we shall assume that the gauge field configuration has been chosen so that the zero mode content on $\Sigma_{4}$ is trivial.

We now study vacua with a non-trivial supersymmetric gauge field configuration in the bulk theory which breaks $S O(12)$ to $S O(10) \times U(1)$. The relevant representations of $S O(12)$ decompose as:

$$
\begin{aligned}
\mathbf{6 6} & \rightarrow 45_{0}+\mathbf{1 0}_{2}+\mathbf{1 0}_{-2}+\mathbf{1}_{0} \\
32 & \rightarrow \mathbf{1 6}_{1}+\overline{\mathbf{1 6}}_{-1} \\
32^{\prime} & \rightarrow \mathbf{1 6}_{-1}+\overline{\mathbf{1 6}}_{+1} \\
\mathbf{1 2} & \rightarrow \mathbf{1 0}_{0}+\mathbf{1}_{2}+\mathbf{1}_{-2} .
\end{aligned}
$$

Again invoking the results of Appendix E, as an example we consider supersymmetric gauge field configurations determined by line bundles of the form:

$$
\mathcal{L}=\mathcal{O}_{S}\left(E_{1}+E_{2}+a E_{3}\right)
$$

where $a$ is a negative integer.

Labelling the the zero modes corresponding to bulk $10_{ \pm 2}$ 's by $\mathbf{1 0}_{ \pm 2}^{(S)}$, their representatives are classified by the bundle valued cohomology groups:

$$
\mathbf{1 0}_{ \pm 2}^{(S)} \in H \frac{1}{\partial}\left(S, \mathcal{L}^{ \pm 2}\right)
$$


The number of massless $10_{ \pm 2}^{(S)}$ 's in the bulk of $S$ is now given by the same index computation used in previous toy models:

$$
\begin{aligned}
& n_{\mathbf{1 0}_{+2}^{(S)}}=2 a^{2}-a+1 \\
& n_{\mathbf{1 0}_{-2}^{(S)}}=2 a^{2}+a+5 .
\end{aligned}
$$

To deduce the matter content along each matter curve, we first specify the homology class of each $\Sigma_{i}$. While it is important to specify the explicit Calabi-Yau fourfold which realizes such a configuration, for illustrative purposes we defer such issues to [19] and assume that the effective class in $H_{2}(S, \mathbb{Z})$ of each matter curve is:

$$
\begin{aligned}
& {\left[\Sigma_{1}\right]=E_{1}} \\
& {\left[\Sigma_{2}\right]=H-E_{1}-E_{2}} \\
& {\left[\Sigma_{3}\right]=3 H-E_{1}-E_{2}}
\end{aligned}
$$

so that $\Sigma_{1}$ and $\Sigma_{2}$ have genus zero and $\Sigma_{3}$ has genus one. ${ }^{22}$ Note that these classes intersect pairwise as $\left[\Sigma_{i}\right] \cdot\left[\Sigma_{j}\right]=+1$ for $i \neq j$. Restricting $\mathcal{L}$ to $\Sigma_{1}$ and $\Sigma_{2}$ yields:

$$
\begin{aligned}
\mathcal{L}_{\Sigma_{1}} & =\mathcal{O}_{\Sigma_{1}}(-1) \\
\mathcal{L}_{\Sigma_{2}} & =\mathcal{O}_{\Sigma_{2}}(+2) .
\end{aligned}
$$

For our present purposes, it is enough to note that $\mathcal{L}_{\Sigma_{3}}$ is a degree two line bundle on $\Sigma_{3}$.

We now present the zero mode content localized along each matter curve. Along $\Sigma_{1}$, the number of massless $\mathbf{1 6}$ 's and $\overline{\mathbf{1 6}}$ 's are classified by the cohomology groups:

$$
\begin{aligned}
& 1 \times \mathbf{1 6}_{-1} \in H \frac{0}{\partial}\left(\Sigma_{1}, K_{\Sigma_{1}}^{1 / 2} \otimes \mathcal{O}_{\Sigma_{1}}(+1)\right)=H \frac{0}{\partial}\left(\Sigma_{1}, \mathcal{O}_{\Sigma_{1}}(0)\right) \\
& 0 \times \overline{\mathbf{1 6}}_{+1} \in H \frac{0}{\partial}\left(\Sigma_{1}, K_{\Sigma_{1}}^{1 / 2} \otimes \mathcal{O}_{\Sigma_{1}}(-1)\right)=H \frac{0}{\partial}\left(\Sigma_{1}, \mathcal{O}_{\Sigma_{1}}(-2)\right)
\end{aligned}
$$

where we have also indicated the multiplicity of each type of massless mode by the overall prefactor. Summarizing, we find a single 16 which descends from $\Sigma_{1}$.

\footnotetext{
${ }^{22}$ The genus $g$ of a smooth curve $C$ in $S$ is given by the intersection-theoretic formula $C \cdot\left(C+K_{S}\right)=$ $2 g-2$
} 
Along $\Sigma_{2}$, the number of $\mathbf{1 6}$ 's and $\overline{\mathbf{1 6}}$ 's are classified by the cohomology groups:

$$
\begin{aligned}
& 2 \times \mathbf{1 6}_{+1} \in H \frac{0}{\partial}\left(\Sigma_{2}, K_{\Sigma_{2}}^{1 / 2} \otimes \mathcal{O}_{\Sigma_{2}}(+2)\right)=H \frac{0}{\partial}\left(\Sigma_{2}, \mathcal{O}_{\Sigma_{2}}(1)\right) \\
& 0 \times \overline{\mathbf{1 6}}_{-1} \in H \frac{0}{\partial}\left(\Sigma_{2}, K_{\Sigma_{2}}^{1 / 2} \otimes \mathcal{O}_{\Sigma_{2}}(-2)\right)=H \frac{0}{\partial}\left(\Sigma_{2}, \mathcal{O}_{\Sigma_{2}}(-3)\right)
\end{aligned}
$$

so that two generations transforming in the $\mathbf{1 6}$ descend from $\Sigma_{2}$.

To compute the dimensions of the relevant cohomology groups for powers of the degree two line bundle $\mathcal{L}_{\Sigma_{3}}$ on $\Sigma_{3}$, we first note that because $K_{\Sigma_{3}}$ is trivial, the Kodaira vanishing theorem implies:

$$
H \frac{0}{\partial}\left(\Sigma_{3}, \mathcal{L}_{\Sigma_{3}}^{-n}\right) \simeq H \frac{1}{\partial}\left(\Sigma_{3}, \mathcal{L}_{\Sigma_{3}}^{n}\right)^{*}=0
$$

when $n>0$. In this particular case, it now follows that either $h^{0}\left(\Sigma_{3}, \mathcal{L}_{\Sigma_{3}}^{m}\right)$ or $h^{1}\left(\Sigma_{3}, \mathcal{L}_{\Sigma_{3}}^{m}\right)$ is zero for $m \neq 0$ so that the index theorem of equation (4.103) in fact counts the total number of zero mode solutions. Along $\Sigma_{3}$, the number of $\mathbf{1}_{ \pm 2}$ 's are classified by the cohomology groups:

$$
\begin{aligned}
4 \times \mathbf{1}_{2} & \in H \frac{0}{\partial}\left(\Sigma_{3}, K_{\Sigma_{3}}^{1 / 2} \otimes \mathcal{L}_{\Sigma_{3}}^{2}\right)=H \frac{0}{\partial}\left(\Sigma_{1}, \mathcal{L}_{\Sigma_{3}}^{2}\right) \\
0 \times \mathbf{1}_{-2} & \in H \frac{0}{\bar{\partial}}\left(\Sigma_{1}, K_{\Sigma_{1}}^{1 / 2} \otimes \mathcal{L}_{\Sigma_{3}}^{-2}\right)=H \frac{0}{\bar{\partial}}\left(\Sigma_{1}, \mathcal{L}_{\Sigma_{3}}^{-2}\right)=0
\end{aligned}
$$

where in computing the multiplicities we have used the fact that:

$$
\int_{\Sigma_{3}} c_{1}\left(\mathcal{L}_{\Sigma_{3}}\right)=\operatorname{deg} \mathcal{L}_{\Sigma_{3}}=2
$$

Even though the $10_{0}$ 's are uncharged under the background gauge field on $\Sigma_{3}$, because $\Sigma_{3}$ is a genus one curve, we also find non-trivial zero mode solutions on $\Sigma_{3}$ which are classified by the cohomology group:

$$
1 \times 10_{0} \in H \frac{0}{\partial}\left(\Sigma_{3}, \mathcal{O}_{\Sigma_{3}}\right) \simeq H \frac{1}{\partial}\left(\Sigma_{3}, \mathcal{O}_{\Sigma_{3}}\right)^{*} \simeq \mathbb{C} .
$$

In the present context, because the $\mathbf{1 0}_{0}$ descends from a single half hypermultiplet on $\Sigma_{3}, H \frac{0}{\partial}\left(\Sigma_{3}, \mathcal{O}_{\Sigma_{3}}\right)$ and $H \frac{1}{\partial}\left(\Sigma_{3}, \mathcal{O}_{\Sigma_{3}}\right)$ classify CPT conjugate particles so that the "chiral matter" is computed by $H \frac{0}{\partial}\left(\Sigma_{3}, \mathcal{O}_{\Sigma_{3}}\right)$. To summarize, the chiral matter content on $\Sigma_{3}$ is given by four singlets and a single 10 . 
We now summarize the contributions to the superpotential of the four-dimensional effective theory:

$$
W_{t o t}=W_{\Sigma \Sigma S}+W_{\Sigma \Sigma \Sigma}
$$

where $W_{\Sigma \Sigma S}$ denotes the contribution from couplings between two fields on a Riemann surface and a bulk field in $S$, and $W_{\Sigma \Sigma \Sigma}$ denotes the contribution from the triple overlap of zero modes localized at the intersection point. The contribution to $W_{\Sigma \Sigma S}$ is:

$$
\begin{aligned}
W_{\Sigma \Sigma S} & =\lambda^{(1)} \mathbf{1 6}_{-1} \times \mathbf{1 6}_{-1} \times \mathbf{1 0}_{+2}^{(S)} \\
& +\lambda_{i j}^{(2)} \mathbf{1 6}_{+1}^{(i)} \times \mathbf{1 6}_{+1}^{(j)} \times \mathbf{1 0}_{-2}^{(S)} \\
& +\lambda_{k}^{(3)} \mathbf{1}_{+2}^{(k)} \times \mathbf{1 0}_{0} \times \mathbf{1 0} 0_{-2}^{(S)}
\end{aligned}
$$

where the superscript on the $\lambda$ 's labels the contribution from each matter curve, and $i, j=1,2$ label the two zero modes in the $\mathbf{1 6}$ localized along $\Sigma_{2}$ and $k=1, \ldots, 4$ labels the four zero modes in the singlet localized along $\Sigma_{3}$. The contribution to $W_{\Sigma \Sigma \Sigma}$ is:

$$
W_{\Sigma \Sigma \Sigma}=\alpha_{i} 16_{-1} \times 16_{+1}^{(i)} \times 10_{0}
$$

where in the above equation, all wave functions for the zero modes are evaluated at the point $p$ and $\alpha_{i}$ includes the contribution from the structure constants of $E_{8}$ restricted to $S O(10)$.

\section{Conclusions}

In this paper we have developed a framework for constructing GUT models from local compactifications of F-theory in terms of a partially twisted eight-dimensional theory with codimension one and two defects. In the presence of a non-trivial gauge bundle, both the pure eight-dimensional theory and theory with defects admit a chiral matter spectrum. When the Kähler surface of the partially twisted theory is a del Pezzo or Hirzebruch surface, non-zero interaction terms require the presence of codimension one defects. In the absence of codimension two defects, the possible interaction terms are greatly limited because two fields localized along a single matter curve must participate in each such Yukawa coupling. More general couplings are possible in the presence of codimension two defects. In this case interaction terms can arise from the 
triple overlap of matter curves at a single point of the bulk gauge theory. In addition to providing explicit examples of various model building ingredients, we have also shown that the degrees of freedom of the partially twisted eight-dimensional theory precisely match to the unfolding of the singularity type of the F-theory geometry. In particular, we have demonstrated that brane recombination for both perturbative and non-perturbatively realized gauge theories in F-theory admits both a consistent gauge theory and geometric interpretation in terms of field vevs with a given pole structure. In the remainder of this Section we discuss some possible applications and extensions of the above work.

Although our ultimate goal is the construction of semi-realistic GUT models, the present investigation has also established a beautiful connection between a partially twisted eight-dimensional theory and the unfolding of geometric singularities in Ftheory. Along these lines, the appearance of fields localized along surface operators, and the presence of point-like "defects" in the partially twisted eight-dimensional theory seem quite natural from the perspective of F-theory. It would be interesting to determine how the intersection of surface operators is described in the four-dimensional topological theory.

In the context of more phenomenological applications, it is also important to study supersymmetry breaking in the present context. While we have restricted attention to supersymmetry preserving vacua which satisfy a modified Hitchin-like system with a source term along a six-dimensional defect, non-supersymmetric instanton solutions of the internal portion of the eight-dimensional theory would correspond to metastable or stable supersymmetry breaking vacua in the four-dimensional effective theory. It would be interesting to develop additional features of such a scenario.

Finally, while for concreteness the toy models presented have all been based on cases where $S$ is a del Pezzo surface, much of the analysis we have presented is of a more general scope and directly applies to a wide class of F-theory compactifications which may not possess a heterotic dual. Anticipating potential applications to GUTs, note that when $h^{2,0}(S) \neq 0$, additional moduli corresponding to the deformation of $S$ in the compactification will in general be present. From the perspective of the GUT group, such fields are charged under the adjoint representation. In the presence of a suitable background flux, these moduli can be frozen to a non-zero vev. These fields can then play the role of more traditional GUT Higgs fields used to break the GUT group down to the Standard Model gauge group. 


\section{Acknowledgements}

We thank B. Andreas, D.R. Morrison, M. Wijnholt and E. Witten for helpful discussions. We would also like to thank the Fifth Simons Workshop in Mathematics and Physics, where this work was initiated. The work of the authors is supported in part by NSF grants PHY-0244821 and DMS-0244464. The research of JJH is also supported by an NSF Graduate Fellowship.

\section{Appendices}

\section{A Review of Hirzebruch and del Pezzo Surfaces}

We now briefly review some properties of Hirzebruch and del Pezzo surfaces. For $n \geq 0$, the middle homology of the Hirzebruch surfaces $\mathbb{F}_{n}$ is generated by the effective classes $f$ and $\sigma$ with intersection pairings:

$$
f \cdot f=0, f \cdot \sigma=1, \sigma \cdot \sigma=-n .
$$

The canonical divisor of $\mathbb{F}_{n}$ is given by:

$$
K_{S}=-c_{1}(S)=-(n+2) f-2 \sigma .
$$

The effective classes correspond to 2-cycles $a f+b \sigma$ such that $a$ and $b$ are both non-negative with at least one non-trivial.

A del Pezzo surface is defined by the condition that $-K_{S}>0$. This condition is satisfied for the surfaces $\mathbb{F}_{0}=\mathbb{P}^{1} \times \mathbb{P}^{1}$ and $d P_{0}=\mathbb{P}^{2}$. There are eight additional del Pezzo surfaces given by blowing up $\mathbb{P}^{2}$ at up to eight points in general position. For $n>0$, The middle homology of the del Pezzo $n\left(d P_{n}\right)$ surface is generated by the hyperplane class $H$ and the exceptional classes $E_{1}, \ldots, E_{n}$ with intersection pairing:

$$
H \cdot H=1, H \cdot E_{i}=0, E_{i} \cdot E_{j}=-\delta_{i j} .
$$


The canonical divisor on $d P_{n}$ is given by:

$$
K_{S}=-c_{1}\left(d P_{n}\right)=-3 H+\sum_{i=1}^{n} E_{i} .
$$

The class of any effective curve $C$ in $d P_{n}$ reduces to a sum over the generators $C_{i}$ of the Kähler cone as:

$$
C=\sum_{i} n_{i} C_{i}
$$

where each $n_{i} \geq 0$. The generators of the Kähler cone for each del Pezzo $n \geq 1$ are well-known in the mathematics literature:

\begin{tabular}{|l|l|}
\hline Surface & Generators \\
\hline$d P_{1}$ & $E_{1}, H-E_{1}$ \\
\hline$d P_{2}$ & $E_{i}, H-\sum_{j=1}^{2} E_{i_{j}}$ \\
\hline$d P_{3}$ & $E_{i}, H-\sum_{j=1}^{2} E_{i_{j}}$ \\
\hline$d P_{4}$ & $E_{i}, H-\sum_{j=1}^{2} E_{i_{j}}$ \\
\hline$d P_{5}$ & $E_{i}, H-\sum_{j=1}^{2} E_{i_{j}}, 2 H-\sum_{j=1}^{5} E_{i_{j}}$ \\
\hline$d P_{6}$ & $E_{i}, H-\sum_{j=1}^{2} E_{i_{j}}, 2 H-\sum_{j=1}^{5} E_{i_{j}}$ \\
\hline$d P_{7}$ & $E_{i}, H-\sum_{j=1}^{2} E_{i_{j}}, 2 H-\sum_{j=1}^{5} E_{i_{j}}, 3 H-2 E_{i}-\sum_{j=1}^{6} E_{i_{j}}$ \\
\hline$d P_{8}$ & $E_{i}, H-\sum_{j=1}^{2} E_{i_{j}}, 2 H-\sum_{j=1}^{5} E_{i_{j}}, 3 H-2 E_{i}-\sum_{j=1}^{6} E_{i_{j}}$, \\
& $4 H-2\left(E_{i}+E_{j}+E_{k}\right)-\sum_{j=1}^{5} E_{i_{j}}$ \\
\hline
\end{tabular}

where all indices are distinct. We note in passing that the number of generators of the Kähler cone for each del Pezzo are 1,2, 3, 6, 10, 16, 27, 56, 240.

A common formula which is used in the text to compute the chiral matter content induced in the presence of a supersymmetric line bundle is given by the Todd genus 
on a surface $S$ :

$$
\int_{S} T d(S)=\int_{S} \frac{c_{1}(S)^{2}+c_{2}(S)}{12}=1-h^{0,1}+h^{0,2}=\chi\left(S, \mathcal{O}_{S}\right)
$$

which is the holomorphic Euler characteristic. On the Hirzebruch and del Pezzo surfaces, $h^{0,1}=h^{0,2}=0$ so that the Todd genus is 1 .

\section{B On-Shell Twisted Supersymmetries of the Eight-Dimensional Theory}

In this appendix we present our conventions for the action of the supercharges $Q_{\alpha}$ and $\bar{Q}_{\dot{\alpha}}$, for $\alpha, \dot{\alpha}=1,2$, on the bosons and fermions of the eight-dimensional, partially twisted Yang-Mills theory. As standard, we denote the action of $Q_{\alpha}$ and $\bar{Q}_{\dot{\alpha}}$ by $\delta_{\alpha}(\cdot)=\left[Q_{\alpha}, \cdot\right\}$ and $\bar{\delta}_{\dot{\alpha}}(\cdot)=\left[\bar{Q}_{\dot{\alpha}}, \cdot\right\}$.

The action by $\delta_{\alpha}$ and $\bar{\delta}_{\dot{\alpha}}$ on the bosons and fermions in the twisted Yang-Mills theory on $\mathbb{R}^{3,1} \times S$ follows immediately from the reduction of the on-shell supersymmetry transformations in the maximally supersymmetric ten-dimensional Yang-Mills theory.

First, we find that the supersymmetry transformations of the eight-dimensional gauge field $A$ and the twisted scalar fields $(\varphi, \bar{\varphi})$ are given by

$$
\begin{array}{lll}
\delta_{\alpha} A_{m}=0, & \bar{\delta}_{\dot{\alpha}} A_{m}=\sqrt{2} \bar{\psi}_{\dot{\alpha} m}, \\
\delta_{\alpha} A_{\bar{m}}=\sqrt{2} \psi_{\alpha \bar{m}}, & \bar{\delta}_{\dot{\alpha}} A_{\bar{m}}=0, \\
\delta_{\alpha} A_{\mu}=i\left(\sigma_{\mu}\right)_{\alpha \dot{\alpha}} \bar{\eta}^{\dot{\alpha}}, & \bar{\delta}_{\dot{\alpha}} A_{\mu}=-i\left(\sigma_{\mu}\right)_{\alpha \dot{\alpha}} \eta^{\alpha}, \\
\delta_{\alpha} \varphi_{m n}=\sqrt{2} \chi_{\alpha m n}, & \bar{\delta}_{\dot{\alpha} \varphi_{m n}}=0, \\
\delta_{\alpha} \bar{\varphi}_{\overline{m n}}=0, & \bar{\delta}_{\dot{\alpha} \bar{\varphi}_{\overline{m n}}}=\sqrt{2} \bar{\chi}_{\dot{\alpha} \overline{m n}} .
\end{array}
$$

Here $A_{\mu}$ for $\mu=0, \ldots, 3$ are the components of the gauge field on $\mathbb{R}^{3,1}$, and $\left(A_{m}, A_{\bar{m}}\right)$ for $m=1,2$ are the components of the gauge field on $S$. Also, $\left(\sigma_{\mu}\right)_{\alpha \dot{\alpha}}$ for $\mu=0, \ldots, 3$ are the standard Pauli matrices which represent the Clifford algebra on $\mathbb{R}^{3,1}$, as defined for instance in Appendix B of [37]. Up to rescalings of the fermions, the supersymmetry transformations in (B.1) are the only possibility consistent with the 
topological twist. Finally, we have included various factors of $\sqrt{2}$ in (B.1) so that our conventions for the effective $\mathcal{N}=1$ supersymmetry algebra on $\mathbb{R}^{3,1}$ agree with the conventions in [37].

Similarly, the on-shell supersymmetry transformations of the fermions are given by

$$
\begin{aligned}
& \delta_{\alpha} \eta_{\beta}=\left(\sigma^{\mu \nu}\right)_{\alpha \beta} F_{\mu \nu}-i \epsilon_{\alpha \beta} \mathcal{D}, \quad \bar{\delta}_{\dot{\alpha}} \eta_{\beta}=0, \\
& \delta_{\alpha} \bar{\eta}_{\dot{\beta}}=0, \quad \bar{\delta}_{\dot{\alpha}} \bar{\eta}_{\dot{\beta}}=\left(\bar{\sigma}^{\mu \nu}\right)_{\dot{\alpha} \dot{\beta}} F_{\mu \nu}+i \epsilon_{\dot{\alpha} \dot{\beta}} \mathcal{D}, \\
& \delta_{\alpha} \psi_{\beta \bar{m}}=\sqrt{2} \epsilon_{\alpha \beta}\left(\bar{\partial}_{A}^{\dagger} \bar{\varphi}\right)_{\bar{m}}, \quad \bar{\delta}_{\dot{\alpha}} \psi_{\beta \bar{m}}=i \sqrt{2}\left(\sigma^{\mu}\right)_{\beta \dot{\alpha}} F_{\mu \bar{m}}, \\
& \delta_{\alpha} \bar{\psi}_{\dot{\beta} m}=i \sqrt{2}\left(\sigma^{\mu}\right)_{\alpha \dot{\beta}} F_{\mu m}, \quad \bar{\delta}_{\dot{\alpha}} \bar{\psi}_{\dot{\beta} m}=\sqrt{2} \epsilon_{\dot{\alpha} \dot{\beta}}\left(\partial_{A}^{\dagger} \varphi\right)_{m}, \\
& \delta_{\alpha} \chi_{\beta m n}=-\sqrt{2} \epsilon_{\alpha \beta} F_{m n}, \quad \bar{\delta}_{\dot{\alpha}} \chi_{\beta m n}=i \sqrt{2}\left(\sigma^{\mu}\right)_{\beta \dot{\alpha}} D_{\mu} \varphi_{m n} \text {, } \\
& \delta_{\alpha} \bar{\chi}_{\dot{\beta} \overline{m n}}=i \sqrt{2}\left(\sigma^{\mu}\right)_{\alpha \dot{\beta}} D_{\mu} \bar{\varphi}_{\overline{m n}}, \quad \bar{\delta}_{\dot{\alpha}} \bar{\chi}_{\dot{\beta} \overline{m n}}=-\sqrt{2} \epsilon_{\dot{\alpha} \dot{\beta}} F_{\overline{m n}} \text {. }
\end{aligned}
$$

Let us explain the notation in (B.2). First, $\mathcal{D}$ is shorthand for the section of $\operatorname{ad}(P)$ on $S$ given by

$$
\mathcal{D}=-\star_{S}\left(\omega \wedge F_{S}+\frac{i}{2}[\varphi, \bar{\varphi}]\right) .
$$

In the above, $\omega$ is the Kähler form on $S$, and $\star_{S}$ denotes the duality operator on $S$ defined by the given Kähler metric. Also, we let $\left.F_{S} \equiv F\right|_{S}$ denote the restriction of the curvature to $S$, so that $\omega \wedge F_{S}$ is a top-form on $S$ upon which $\star_{S}$ naturally acts. The overall sign in (B.3) is just a convention.

Throughout the paper, we use $\left(\partial_{A}, \bar{\partial}_{A}\right)$ to indicate the $(1,0)$ and $(0,1)$ components of the covariant derivative defined by the gauge field on $S$, and we let $\left(\partial_{A}^{\dagger}, \bar{\partial}_{A}^{\dagger}\right)$ be the adjoint operators defined using $\star_{S}$. Explicitly, $\bar{\partial}_{A}^{\dagger}=-\star_{S} \bar{\partial}_{A^{\star}}$, and similarly for $\partial_{A}^{\dagger}$. In local coordinates $\left(s^{m}, \bar{s}^{\bar{m}}\right)$ on $S,\left(\partial_{A}^{\dagger} \varphi\right)_{m}=g^{n \bar{n}}\left(\bar{\partial}_{A}\right)_{\bar{n}} \varphi_{m n}$, where $g_{n \bar{n}}$ is the Kähler metric.

Finally, we use $D_{\mu}$ to indicate the covariant derivative defined by the gauge field along $\mathbb{R}^{3,1}$, and we normalize the self-dual and anti-self-dual projection operators $\left(\sigma^{\mu \nu}\right)_{\alpha \beta}$ and $\left(\bar{\sigma}^{\mu \nu}\right)_{\dot{\alpha} \dot{\beta}}$ appearing in (B.2) as in [37]. Also, $\epsilon_{\alpha \beta}$ and $\epsilon_{\dot{\alpha} \dot{\beta}}$ are the usual anti-symmetric tensors on two indices. 


\section{Partially Twisted Action of the Seven-Brane Theory}

In this Appendix we determine the Lagrangian of the partially twisted Yang-Mills theory on $\mathbb{R}^{3,1} \times S$ with gauge group $G_{S}$. In the limit where the fields of the partially twisted theory are independent of the coordinates on $\mathbb{R}^{3,1}$, the reduction to $S$ is given by the twisted version of four-dimensional, $\mathcal{N}=4$ supersymmetric Yang-Mills theory studied in [24]. One purpose of the present Appendix is to explicitly demonstrate the quasi-topological nature of the partially twisted eight-dimensional theory. Other twisted eight-dimensional theories on $\operatorname{Spin}(7)$ manifolds and Calabi-Yau fourfolds have been studied for instance in $[38,39]$.

The supersymmetric Lagrangian for the twisted Yang-Mills theory on $\mathbb{R}^{3,1} \times S$ is most conveniently written once we pass to a (mostly) off-shell formulation of the supersymmetry algebra. The existence of an off-shell formulation of the supersymmetry transformations in (B.1) and (B.2) is not so surprising, since the off-shell formulation is modeled on the standard off-shell formulation of $\mathcal{N}=1$ supersymmetry in four dimensions.

\section{Auxiliary Fields}

We first introduce auxiliary bosonic fields $(\mathcal{G}, \mathcal{H}, \mathcal{D})$. Here $\mathcal{G}=\mathcal{G}_{\bar{m}} d \bar{s}^{\bar{m}}$ is a complex boson which transforms on $S$ as a section of $\bar{\Omega}_{S}^{1} \otimes \operatorname{ad}(P)$, and $\mathcal{H}=\mathcal{H}_{m n} d s^{m} \wedge d s^{n}$

is another complex boson which transforms on $S$ as a section of $\Omega_{S}^{2} \otimes \operatorname{ad}(P)$. From the perspective of the four-dimensional effective theory on $\mathbb{R}^{3,1}, \mathcal{G}$ and $\mathcal{H}$ are the auxiliary components of $\mathcal{N}=1$ chiral superfields

$$
\begin{aligned}
\mathbf{A}_{\bar{m}} & =A_{\bar{m}}+\sqrt{2} \theta \psi_{\bar{m}}+\theta \theta \mathcal{G}_{\bar{m}}+\cdots, \\
\boldsymbol{\Phi}_{m n} & =\varphi_{m n}+\sqrt{2} \theta \chi_{m n}+\theta \theta \mathcal{H}_{m n}+\cdots,
\end{aligned}
$$

where we use '.. ' to indicate the usual higher-order terms in the chiral superfields. While the presentation in (C.1) is convenient for describing certain properties of the four-dimensional effective theory, we note that $A_{\bar{m}}$ does not transform covariantly under arbitrary gauge transformations on $\mathbb{R}^{3,1} \times S$ but rather only under those which are constant along $S$. As a result, we will have to be careful about eight-dimensional gauge-invariance when we construct the action. 
Along with $\mathcal{G}$ and $\mathcal{H}$, we also introduce the conjugate bosons $\overline{\mathcal{G}}=\overline{\mathcal{G}}_{m} d s^{m}$ and $\overline{\mathcal{H}}=\overline{\mathcal{H}}_{\overline{m n}} d \bar{s}^{\bar{m}} \wedge d \bar{s}^{\bar{n}}$ which transform as sections of $\Omega_{S}^{1} \otimes \operatorname{ad}(P)$ and $\bar{\Omega}_{S}^{2} \otimes \operatorname{ad}(P)$, respectively. These bosons appear as the auxiliary components in anti-chiral superfields conjugate to those in (C.1).

We finally introduce a real scalar field $\mathcal{D}$ which transforms on $S$ as a section of $\operatorname{ad}(P)$. In the four-dimensional effective theory, $\mathcal{D}$ is the auxiliary field of the $\mathcal{N}=1$ vector multiplet, appearing in WZ gauge as

$$
V=-\theta \sigma^{\mu} \bar{\theta} A_{\mu}+i \theta \theta \bar{\theta} \bar{\eta}-i \overline{\theta \theta} \theta \eta+\frac{1}{2} \theta \theta \overline{\theta \theta} \mathcal{D} .
$$

Eventually, the auxiliary scalar field $\mathcal{D}$ in $(\mathrm{C} .2)$ will be identified on-shell with the expression in (B.3).

In terms of the auxiliary bosons $(\mathcal{G}, \mathcal{H}, \mathcal{D})$, the supersymmetry transformations (B.2) of the twisted fermions are now given by

$$
\begin{aligned}
& \delta_{\alpha} \eta_{\beta}=\left(\sigma^{\mu \nu}\right)_{\alpha \beta} F_{\mu \nu}-i \epsilon_{\alpha \beta} \mathcal{D}, \quad \bar{\delta}_{\dot{\alpha}} \eta_{\beta}=0, \\
& \delta_{\alpha} \bar{\eta}_{\dot{\beta}}=0, \quad \bar{\delta}_{\dot{\alpha}} \bar{\eta}_{\dot{\beta}}=\left(\bar{\sigma}^{\mu \nu}\right)_{\dot{\alpha} \dot{\beta}} F_{\mu \nu}+i \epsilon_{\dot{\alpha} \dot{\beta}} \mathcal{D}, \\
& \delta_{\alpha} \psi_{\beta \bar{m}}=-\sqrt{2} \epsilon_{\alpha \beta} \mathcal{G}_{\bar{m}}, \quad \bar{\delta}_{\dot{\alpha}} \psi_{\beta \bar{m}}=i \sqrt{2}\left(\sigma^{\mu}\right)_{\beta \dot{\alpha}} F_{\mu \bar{m}}, \\
& \delta_{\alpha} \bar{\psi}_{\dot{\beta} m}=i \sqrt{2}\left(\sigma^{\mu}\right)_{\alpha \dot{\beta}} F_{\mu m}, \quad \bar{\delta}_{\dot{\alpha}} \bar{\psi}_{\dot{\beta} m}=-\sqrt{2} \epsilon_{\dot{\alpha} \dot{\beta}} \overline{\mathcal{G}}_{m}, \\
& \delta_{\alpha} \chi_{\beta m n}=-\sqrt{2} \epsilon_{\alpha \beta} \mathcal{H}_{m n}, \quad \bar{\delta}_{\dot{\alpha}} \chi_{\beta m n}=i \sqrt{2}\left(\sigma^{\mu}\right)_{\beta \dot{\alpha}} D_{\mu} \varphi_{m n}, \\
& \delta_{\alpha} \bar{\chi}_{\dot{\beta} \overline{m n}}=i \sqrt{2}\left(\sigma^{\mu}\right)_{\alpha \dot{\beta}} D_{\mu} \bar{\varphi}_{\overline{m n}}, \quad \bar{\delta}_{\dot{\alpha}} \bar{\chi}_{\dot{\beta} \overline{m n}}=-\sqrt{2} \epsilon_{\dot{\alpha} \dot{\beta}} \overline{\mathcal{H}}_{\overline{m n}} .
\end{aligned}
$$

Ideally, the supersymmetry transformations of the auxiliary bosons $(\mathcal{G}, \mathcal{H}, \mathcal{D})$ are determined by the condition that $\delta_{\alpha}$ and $\bar{\delta}_{\dot{\alpha}}$ satisfy the $\mathcal{N}=1$ supersymmetry algebra

$$
\left\{\delta_{\alpha}, \delta_{\beta}\right\}=\left\{\bar{\delta}_{\dot{\alpha}}, \bar{\delta}_{\dot{\beta}}\right\}=0, \quad\left\{\delta_{\alpha}, \bar{\delta}_{\dot{\alpha}}\right\}=2 i\left(\sigma^{\mu}\right)_{\alpha \dot{\alpha}} D_{\mu} .
$$

However, as is well-known, the algebra in (C.4) cannot be realized off-shell on the $\mathcal{N}=1$ vector multiplet in WZ gauge. We know of two ways to work around this problem, such that we either preserve manifest supersymmetry or manifest gaugeinvariance at all stages of the computation.

One strategy suggested in $[40,41,42]$ is to treat the eight-dimensional fields of the 
partially twisted theory as a collection of four-dimensional fields on $\mathbb{R}^{3,1}$ labelled by coordinates on $S$. Organizing these four-dimensional fields into $\mathcal{N}=1$ superfields, the superspace action for the partially twisted Yang-Mills theory on $\mathbb{R}^{3,1} \times S$ manifestly preserves supersymmetry. However, the superspace description of the twisted Yang-Mills action on $\mathbb{R}^{3,1} \times S$ also obscures the higher-dimensional gauge invariance of the theory.

Because we prefer to maintain manifest gauge-invariance over manifest supersymmetry, in this Appendix we pursue an alternative strategy. Rather than ask for an off-shell formulation of the full $\mathcal{N}=1$ supersymmetry algebra in (C.4), we consider only the simpler algebra

$$
\left\{\bar{\delta}_{\dot{\alpha}}, \bar{\delta}_{\dot{\beta}}\right\}=0
$$

The simple algebra in (C.5) does close off-shell when the auxiliary bosons $(\mathcal{G}, \mathcal{H}, \mathcal{D})$ transform as

$$
\begin{aligned}
& \bar{\delta}_{\dot{\alpha}} \mathcal{G}_{\bar{m}}=i \sqrt{2}\left(\sigma^{\mu}\right)_{\alpha \dot{\alpha}} D_{\mu} \psi_{\frac{\alpha}{m}}+2\left(\bar{\partial}_{A} \bar{\eta}_{\dot{\alpha}}\right)_{\bar{m}}, \\
& \bar{\delta}_{\dot{\alpha}} \overline{\mathcal{G}}_{m}=0, \\
& \bar{\delta}_{\dot{\alpha}} \mathcal{H}_{m n}=i \sqrt{2}\left(\sigma^{\mu}\right)_{\alpha \dot{\alpha}} D_{\mu} \chi_{m n}^{\alpha}+2\left[\varphi_{m n}, \bar{\eta}_{\dot{\alpha}}\right], \\
& \bar{\delta}_{\dot{\alpha}} \overline{\mathcal{H}}_{\overline{m n}}=0 \\
& \bar{\delta}_{\dot{\alpha}} \mathcal{D}=-\left(\sigma^{\mu}\right)_{\alpha \dot{\alpha}} D_{\mu} \eta^{\alpha} .
\end{aligned}
$$

The supersymmetry transformations in (C.6) are merely covariant versions of the usual transformations for the auxiliary bosons in the $\mathcal{N}=1$ chiral and vector multiplets.

For future reference, we also recall the conjugate supersymmetry transformations under $\delta_{\alpha}$

$$
\begin{aligned}
& \delta_{\alpha} \mathcal{G}_{\bar{m}}=0, \\
& \delta_{\alpha} \overline{\mathcal{G}}_{m}=i \sqrt{2}\left(\sigma^{\mu}\right)_{\alpha \dot{\alpha}} D_{\mu} \bar{\psi}_{m}^{\dot{\alpha}}+2\left(\partial_{A} \eta_{\alpha}\right)_{m}, \\
& \delta_{\alpha} \mathcal{H}_{m n}=0 \\
& \delta_{\alpha} \overline{\mathcal{H}}_{\overline{m n}}=i \sqrt{2}\left(\sigma^{\mu}\right)_{\alpha \dot{\alpha}} D_{\mu} \bar{\chi}_{\overline{m n}}^{\dot{\alpha}}+2\left[\bar{\varphi}_{\overline{m n}}, \eta_{\alpha}\right], \\
& \delta_{\alpha} \mathcal{D}=-\left(\sigma^{\mu}\right)_{\alpha \dot{\alpha}} D_{\mu} \bar{\eta}^{\dot{\alpha}} .
\end{aligned}
$$


Constructing the Supersymmetric Action

Despite the fact that (C.3) and (C.6) only provide an off-shell realization for the simple algebra involving $\bar{\delta}_{\dot{\alpha}}$ in (C.5), the partially twisted action on $\mathbb{R}^{3,1} \times S$ must be invariant under both $\delta_{\alpha}$ and $\bar{\delta}_{\dot{\alpha}}$. To construct an action invariant under both types of transformations, we use the fact that $\delta_{\alpha}$ is conjugate to $\bar{\delta}_{\dot{\alpha}}$. As a result, any action $I_{S}$ which is both real and annihilated by $\delta_{\alpha}$ is automatically annihilated by $\bar{\delta}_{\dot{\alpha}}$.

At weak coupling, the partially twisted action serves to enforce the BPS equations of motion described in subsection 3.3.1. In order to make the quasi-topological nature of the partially twisted theory manifest, we organize the action into three types of terms. The first two types of terms correspond to $\bar{\delta}^{2}$ or $\bar{\delta}_{\dot{\alpha}}$ exact operators so that by construction such terms are annihilated by $\bar{\delta}_{\dot{\alpha}}$. In terms of the effective theory in four dimensions, the final class of terms corresponds to integrating the superpotential over chiral superspace. These terms are $\bar{\delta}_{\dot{\alpha}}$ closed but not exact.

We now present the $\bar{\delta}^{2}$ trivial terms of $I_{S}$. Many of these terms contribute to terms in the action serve to enforce the BPS equations motion. To this end, we first introduce the operator

$$
\mathcal{O}^{(1)}=\frac{1}{4} \int_{\mathbb{R}^{3,1} \times S} d^{4} x \operatorname{Tr}(\mathcal{H} \wedge \bar{\varphi}) .
$$

We now compute

$$
\begin{aligned}
& I_{1}=\bar{\delta}^{2} \mathcal{O}^{(1)}, \\
& =\int_{\mathbb{R}^{3,1} \times S} d^{4} x \operatorname{Tr}\left(\mathcal{H} \wedge \overline{\mathcal{H}}-D^{\mu} \bar{\varphi} \wedge D_{\mu} \varphi+i[\varphi, \bar{\varphi}] \mathcal{D}+\right. \\
& \left.+i D_{\mu} \chi^{\alpha}\left(\sigma^{\mu}\right)_{\alpha \dot{\alpha}} \bar{\chi}^{\dot{\alpha}}-\sqrt{2} \eta^{\alpha}\left[\bar{\varphi}, \chi_{\alpha}\right]-\sqrt{2} \bar{\eta}_{\dot{\alpha}}\left[\varphi, \bar{\chi}^{\dot{\alpha}}\right]\right) .
\end{aligned}
$$

As expected, the integrands of (C.8) and (C.9) are differential forms of top-degree on $S$.

The role of $I_{1}$ is to produce a Gaussian action for $\mathcal{H}$ and $\overline{\mathcal{H}}$, along with certain kinetic terms for $\varphi$ and the fermions. In order to reproduce the relations $F_{S}^{(2,0)}=0$ and $F_{S}^{(0,2)}=0$, we next introduce source terms for $\mathcal{H}$ and $\overline{\mathcal{H}}$ which enforce these conditions when $\mathcal{H}$ and $\overline{\mathcal{H}}$ are integrated out. One of these source terms is also 
$\bar{\delta}^{2}$-trivial and descends from the operator

$$
\mathcal{O}^{(2)}=-\frac{1}{4} \int_{\mathbb{R}^{3,1} \times S} d^{4} x \operatorname{Tr}\left(F_{S}^{(2,0)} \wedge \bar{\varphi}\right),
$$

so that its contribution to the action is

$$
\begin{aligned}
I_{2}= & \bar{\delta}^{2} \mathcal{O}^{(2)}, \\
=-\int_{\mathbb{R}^{3,1} \times S} d^{4} x \operatorname{Tr}( & F_{S}^{(2,0)} \wedge \overline{\mathcal{H}}+\overline{\mathcal{G}} \wedge \partial_{A} \bar{\varphi}+ \\
& \left.\quad+\bar{\chi}_{\dot{\alpha}} \wedge \partial_{A} \bar{\psi}^{\dot{\alpha}}-\frac{1}{2} \bar{\psi}_{\dot{\alpha}}\left[\bar{\varphi}, \bar{\psi}^{\dot{\alpha}}\right]\right) .
\end{aligned}
$$

The final $\bar{\delta}^{2}$-trivial term which contributes to $I_{S}$ serves to reproduce the standard action for the four-dimensional $\mathcal{N}=1$ vector multiplet in $\mathbb{R}^{3,1}$. Explicitly, this term descends from the operator

$$
\mathcal{O}^{(3)}=\frac{1}{8} \int_{\mathbb{R}^{3,1} \times S} d^{4} x \omega \wedge \omega \operatorname{Tr}\left(\bar{\eta}_{\dot{\alpha}} \bar{\eta}^{\dot{\alpha}}\right) .
$$

We note that because $\bar{\eta}_{\dot{\alpha}}$ is a zero-form on $S$, we must introduce two powers of the Kähler form $\omega$ on $S$ to obtain an appropriate measure for the integral in (C.12). As completely standard for the $\mathcal{N}=1$ vector multiplet in four dimensions, we obtain

$$
\begin{aligned}
I_{3}=\bar{\delta}^{2} \mathcal{O}^{(3)}, & \\
=\int_{\mathbb{R}^{3,1} \times S} d^{4} x \omega \wedge \omega & \operatorname{Tr}\left(\frac{1}{2} \mathcal{D}^{2}-\frac{1}{4} F^{\mu \nu} F_{\mu \nu}-\frac{i}{8} \epsilon^{\mu \nu \rho \sigma} F_{\mu \nu} F_{\rho \sigma}+\right. \\
& \left.+i\left(\sigma^{\mu}\right)_{\alpha \dot{\alpha}} D_{\mu} \eta^{\alpha} \bar{\eta}^{\dot{\alpha}}\right) .
\end{aligned}
$$

In (C.13), $\epsilon^{\mu \nu \rho \sigma}$ is the anti-symmetric tensor associated to the Pontryagin density for the curvature on $\mathbb{R}^{3,1}$. While this naively appears to fix a specific value of the $\theta_{Y M}$ angle in the four-dimensional effective theory, we note that because the Pontryagin density is a topological term, it is automatically supersymmetric. Therefore, we may add an additional topological term of this type to the quasi-topological action in 
order to achieve a four-dimensional effective theory with an arbitrary $\theta_{Y M}$ angle.

Besides terms which are $\bar{\delta}^{2}$-trivial, the action $I_{S}$ also contains a set of terms of the form

$$
I_{4}=\epsilon^{\dot{\alpha} \dot{\beta}} \bar{\delta}_{\dot{\alpha}} \mathcal{O}_{\dot{\beta}}^{(4)}
$$

where $\mathcal{O}_{\dot{\beta}}^{(4)}$ for $\dot{\beta}=1,2$ is again a gauge-invariant functional of the fields. In order that $\bar{\delta}_{\dot{\alpha}}$ annihilate $I_{4}$, the functional $\mathcal{O}_{\dot{\beta}}^{(4)}$ cannot be arbitrary but must satisfy

$$
\bar{\delta}_{\dot{\alpha}} \mathcal{O}_{\dot{\beta}}^{(4)}=-\bar{\delta}_{\dot{\beta}} \mathcal{O}_{\dot{\alpha}}^{(4)}
$$

With a little bit of calculation, one can check that the following choice for $\mathcal{O}_{\dot{\beta}}^{(4)}$ satisfies (C.15),

$$
\mathcal{O}_{\dot{\beta}}^{(4)}=-\frac{i}{\sqrt{2}} \int_{\mathbb{R}^{3,1} \times S} d^{4} x \omega \wedge \operatorname{Tr}\left(\bar{\psi}_{\dot{\beta}} \wedge \mathcal{G}-i F_{S \mu}^{(1,0)}\left(\sigma^{\mu}\right)_{\alpha \dot{\beta}} \wedge \psi^{\alpha}-\sqrt{2} F_{S}^{(1,1)} \bar{\eta}_{\dot{\beta}}\right)
$$

where $F_{S \mu}^{(1,0)}=F_{\mu m} d s^{m}$ is determined by the mixed components of the curvature on $\mathbb{R}^{3,1} \times S$. Given the first term appearing in $\mathcal{O}_{\dot{\beta}}^{(4)}$, the coefficients of the remaining two terms are fixed uniquely by the anti-symmetry condition in (C.15).

Of course, one might ask how we arrived at (C.16). The significance of (C.16) is that the corresponding expression for $I_{4}$ contains a term which is quadratic in the auxiliary fields $\mathcal{G}$ and $\overline{\mathcal{G}}$,

$$
\begin{array}{rl}
I_{4}=\int_{\mathbb{R}^{3,1} \times S} d^{4} x & 2 \omega \wedge \operatorname{Tr}\left(i \mathcal{G} \wedge \overline{\mathcal{G}}-i F_{S}^{(1,0) \mu} \wedge F_{S \mu}^{(0,1)}+F_{S}^{(1,1)} \mathcal{D}+\right. \\
& \left.+D_{\mu} \bar{\psi}^{\dot{\alpha}}\left(\sigma^{\mu}\right)_{\alpha \dot{\alpha}} \wedge \psi^{\alpha}-i \sqrt{2} \bar{\psi}_{\dot{\alpha}} \wedge \bar{\partial}_{A} \bar{\eta}^{\dot{\alpha}}-i \sqrt{2} \partial_{A} \eta^{\alpha} \wedge \psi_{\alpha}\right) .
\end{array}
$$

From the perspective of the $\mathcal{N}=1$ supersymmetric theory on $\mathbb{R}^{3,1}$, the terms in $I_{4}$ give rise to D-terms in the low-energy effective action. However, we do not know of any way to write $I_{4}$ in the form $I_{4}=\bar{\delta}^{2} \mathcal{O}^{\prime}$ for any gauge-invariant expression $\mathcal{O}^{\prime}$. This feature is presumably related to the difficulties first encountered in [40] in providing a fully local gauge-invariant description in $\mathcal{N}=1$ superspace for the ten-dimensional super-Yang-Mills action. 
The final term which appears in $I_{S}$ is simply the conjugate to $I_{2}$ in (C.11), which we denote by

$$
W_{S}=-\int_{\mathbb{R}^{3,1} \times S} d^{4} x \operatorname{Tr}\left(F_{S}^{(0,2)} \wedge \mathcal{H}+\mathcal{G} \wedge \bar{\partial}_{A} \varphi+\chi^{\alpha} \wedge \bar{\partial}_{A} \psi_{\alpha}+\frac{1}{2} \psi^{\alpha}\left[\varphi, \psi_{\alpha}\right]\right) .
$$

As one can check, though $W_{S}$ is not $\bar{\delta}_{\dot{\alpha}}$-exact, $W_{S}$ is nonetheless annihilated by $\bar{\delta}_{\dot{\alpha}}$. Our notation for $W_{S}$ is no accident, since $W_{S}$ can be written as an integral over superspace of the superpotential for the twisted Yang-Mills theory on $\mathbb{R}^{3,1} \times S$,

$$
W_{S}=-\int_{\mathbb{R}^{3,1} \times S} d^{4} x d^{2} \theta \operatorname{Tr}\left(\mathbf{F}_{S}^{(0,2)} \wedge \boldsymbol{\Phi}\right)
$$

Here $\boldsymbol{\Phi}$ is the chiral superfield with lowest bosonic component $\varphi$ as introduced in (C.1), and $\mathbf{F}_{S}^{(0,2)}$ is the chiral superfield with lowest bosonic component $F_{S}^{(0,2)}$. Explicitly, in terms of the chiral superfield $\mathbf{A}_{\bar{m}}$ in (C.1),

$$
\begin{aligned}
\mathbf{F}_{S}^{(0,2)} & =\bar{\partial} \mathbf{A}+\mathbf{A} \wedge \mathbf{A} \\
& =F_{S}^{(0,2)}+\sqrt{2} \theta \bar{\partial}_{A} \psi+\theta \theta\left(\bar{\partial}_{A} \mathcal{G}-\frac{1}{2}\left[\psi^{\alpha}, \psi_{\alpha}\right]\right)+\cdots
\end{aligned}
$$

Of all the terms that will appear in $I_{S}$, the terms in (C.18) are most important, since these terms determine the effective superpotential on $\mathbb{R}^{3,1}$ which describes F-theory compactified on $X$.

The supersymmetric action $I_{S}$ for the partially twisted Yang-Mills theory on $\mathbb{R}^{3,1} \times S$ is now given by

$$
\begin{aligned}
I_{S} & =I_{1}+I_{2}+I_{3}+I_{4}+W_{S} \\
& =\int_{\mathbb{R}^{3,1} \times S} d^{4} x \operatorname{Tr}\left[\omega \wedge \omega\left(\frac{1}{2} \mathcal{D}^{2}-\frac{1}{4} F^{\mu \nu} F_{\mu \nu}\right)-2 i \omega \wedge F_{S \mu}^{(1,0)} \wedge F_{S}^{(0,1) \mu}-D^{\mu} \bar{\varphi} \wedge D_{\mu} \varphi+\right. \\
& +2 i \omega \wedge \mathcal{G} \wedge \overline{\mathcal{G}}+\mathcal{H} \wedge \overline{\mathcal{H}}-F_{A}^{(2,0)} \wedge \overline{\mathcal{H}}-F_{S}^{(0,2)} \wedge \mathcal{H}-\overline{\mathcal{G}} \wedge \partial_{A} \bar{\varphi}-\mathcal{G} \wedge \bar{\partial}_{A} \varphi+ \\
& \left.+2\left(\omega \wedge F_{S}^{(1,1)}+\frac{i}{2}[\varphi, \bar{\varphi}]\right) \mathcal{D}\right]+\cdots .
\end{aligned}
$$


This action deserves a number of comments. As indicated by the '...', we omit from (C.21) terms involving fermions as well as the Pontryagin density on $\mathbb{R}^{3,1}$ which appears in (C.13). Modulo the Pontryagin term, $I_{S}$ is purely real. Since $\delta_{\alpha}$ and $\bar{\delta}_{\dot{\alpha}}$ annihilate this term, it now follows that the action $I_{S}$ is annihilated by $\delta_{\alpha}$ and $\bar{\delta}_{\dot{\alpha}}$ as claimed.

By the same token, we are free to add to $I_{S}$ any other topological terms involving characteristic classes of the surface $S$ and the $G_{S^{-}}$-bundle $P$. Along with the effective $\theta_{Y M}$-angle on $\mathbb{R}^{3,1}$, the coefficients of such terms are fixed by closed string moduli which appear as parameters in the local F-theory backgrounds under consideration. Such topological terms will not play a role in the present discussion.

Finally, if we integrate out the auxiliary bosons appearing in (C.21), the offshell supersymmetry algebra in (C.3) reduces to the on-shell algebra in (B.2). By construction, the on-shell action then enforces the F- and D-term supersymmetry conditions at weak-coupling. Finally, as is more or less apparent and dictated by supersymmetry, the on-shell action derived from (C.21) provides a twisted version of the maximally supersymmetric Yang-Mills action on $\mathbb{R}^{3,1} \times S$.

\section{Partially Twisted Action of the Six-Dimensional Defect Theory}

In this Appendix we present the action for a six-dimensional hypermultiplet charged under a gauge group $G_{S} \times G_{S^{\prime}}$ associated with the partially twisted six-dimensional defect theory which originates from the intersection of seven-branes along $\mathbb{R}^{3,1} \times \Sigma$. As opposed to most topological field theories, the on-shell supersymmetry transformations of the fields do not descend directly from a reduction of super Yang-Mills theory in ten dimensions. For this reason, it is convenient to treat the six-dimensional defect theory in a superspace formalism which preserves four-dimensional $\mathcal{N}=1$ offshell supersymmetry. Indeed, in this Appendix we will effectively reverse the usual order of discussion by instead using the off-shell supersymmetry transformations to determine the on-shell transformations of fields in the six- and eight-dimensional theories.

In the spirit of $[40,41,42]$, we now treat the six-dimensional fields of the defect theory as a collection of four-dimensional fields labelled by points of $\Sigma$. In terms of the 
four-dimensional effective theory, the six-dimensional hypermultiplet organizes into two collections of $\mathcal{N}=1$ chiral multiplets $(\sigma, \lambda, \mathcal{K})$ and $\left(\sigma^{c}, \lambda^{c}, \mathcal{K}^{c}\right)$ which transform in dual representations of $G_{S} \times G_{S^{\prime}}$. The off-shell supersymmetry transformations of the bosonic degrees of freedom are given by the usual expressions for an $\mathcal{N}=1$ chiral multiplet. As for the eight-dimensional theory, we follow the conventions of [37]:

$$
\begin{array}{ll}
\delta_{\alpha} \sigma=\sqrt{2} \lambda_{\alpha}, & \bar{\delta}_{\dot{\alpha}} \sigma=0, \\
\delta_{\alpha} \bar{\sigma}=0, & \bar{\delta}_{\dot{\alpha}} \bar{\sigma}=\sqrt{2} \bar{\lambda}_{\dot{\alpha}}, \\
\delta_{\alpha} \sigma^{c}=\sqrt{2} \lambda_{\alpha}^{c}, & \bar{\delta}_{\dot{\alpha}} \sigma^{c}=0, \\
\delta_{\alpha} \bar{\sigma}^{c}=0, & \bar{\delta}_{\dot{\alpha}} \bar{\sigma}^{c}=\sqrt{2} \bar{\lambda}_{\dot{\alpha}}^{c} .
\end{array}
$$

Similarly, the fermions transform as:

$$
\begin{aligned}
& \delta_{\alpha} \lambda_{\beta}=-\sqrt{2} \epsilon_{\alpha \beta} \mathcal{K}, \\
& \bar{\delta}_{\dot{\alpha}} \lambda_{\beta}=i \sqrt{2}\left(\sigma^{\mu}\right)_{\beta \dot{\alpha}} D_{\mu} \sigma, \\
& \delta_{\alpha} \bar{\lambda}_{\dot{\beta}}=i \sqrt{2}\left(\sigma^{\mu}\right)_{\alpha \dot{\beta}} D_{\mu} \bar{\sigma}, \\
& \bar{\delta}_{\dot{\alpha}} \bar{\lambda}_{\dot{\beta}}=-\sqrt{2} \epsilon_{\dot{\alpha} \dot{\beta}} \overline{\mathcal{K}}, \\
& \delta_{\alpha} \lambda_{\beta}^{c}=-\sqrt{2} \epsilon_{\alpha \beta} \mathcal{K}^{c}, \\
& \bar{\delta}_{\dot{\alpha}} \lambda_{\beta}^{c}=i \sqrt{2}\left(\sigma^{\mu}\right)_{\beta \dot{\alpha}} D_{\mu} \sigma^{c}, \\
& \delta_{\alpha} \bar{\lambda}_{\dot{\beta}}=i \sqrt{2}\left(\sigma^{\mu}\right)_{\alpha \dot{\beta}} D_{\mu} \overline{\sigma^{c}} \text {, } \\
& \bar{\delta}_{\dot{\alpha}} \bar{\lambda}_{\dot{\beta}}=-\sqrt{2} \epsilon_{\dot{\alpha} \dot{\beta}} \overline{\mathcal{K}^{c}} \text {. }
\end{aligned}
$$

Finally, the auxiliary fields transform as:

$$
\begin{array}{ll}
\delta_{\alpha} \mathcal{K}=0, & \bar{\delta}_{\dot{\alpha}} \mathcal{K}=i \sqrt{2}\left(\sigma^{\mu}\right)_{\alpha \dot{\alpha}} D_{\mu} \lambda^{\alpha}+2\left[\sigma, \bar{\eta}_{\dot{\alpha}}\right], \\
\delta_{\alpha} \overline{\mathcal{K}}=i \sqrt{2}\left(\sigma^{\mu}\right)_{\alpha \dot{\alpha}} D_{\mu} \bar{\lambda}^{\dot{\alpha}}+2\left[\bar{\sigma}, \eta_{\alpha}\right], & \bar{\delta}_{\dot{\alpha}} \overline{\mathcal{K}}=0, \\
\delta_{\alpha} \mathcal{K}^{c}=0, & \bar{\delta}_{\dot{\alpha}} \mathcal{K}^{c}=i \sqrt{2}\left(\sigma^{\mu}\right)_{\alpha \dot{\alpha}} D_{\mu} \lambda^{c \alpha}+2\left[\sigma^{c}, \bar{\eta}_{\dot{\alpha}}\right], \\
\delta_{\alpha} \overline{\mathcal{K}^{c}}=i \sqrt{2}\left(\sigma^{\mu}\right)_{\alpha \dot{\alpha}} D_{\mu} \bar{\lambda}^{\dot{\alpha}}+2\left[\bar{\sigma}^{c}, \eta_{\alpha}\right], & \bar{\delta}_{\dot{\alpha}} \overline{\mathcal{K}^{c}}=0 .
\end{array}
$$

Organizing these fields into $\mathcal{N}=1$ chiral superfields and a background vector superfield which descends from the bulk eight-dimensional theory on $S$, we have:

$$
\begin{aligned}
\boldsymbol{\Lambda} & =\sigma+\sqrt{2} \theta \lambda+\theta \theta \mathcal{K}+\cdots \\
\boldsymbol{\Lambda}^{c} & =\sigma^{c}+\sqrt{2} \theta \lambda^{c}+\theta \theta \mathcal{K}+\cdots \\
V & =-\theta \sigma^{\mu} \bar{\theta} A_{\mu}+i \theta \theta \bar{\theta} \bar{\eta}-i \overline{\theta \theta} \theta \eta+\frac{1}{2} \theta \theta \overline{\theta \theta} \mathcal{D}
\end{aligned}
$$


where for notational simplicity we have presented the background vector multiplet in WZ gauge. There is a similar background vector superfield $V^{\prime}$ which descends from the bulk theory on $\mathbb{R}^{3,1} \times S^{\prime}$. Finally, to present gauge covariant interactions in the internal directions, we also introduce the gauge covariant derivative superfield defined in equation (4.36) which we reproduce here for the convenience of the reader:

$$
\begin{aligned}
\bar{\partial}_{\mathbf{A}+\mathbf{A}^{\prime}} & =\bar{\partial}+\mathbf{A}+\mathbf{A}^{\prime} \\
& =\bar{\partial}_{A+A^{\prime}}+\sqrt{2} \theta\left(\psi+\psi^{\prime}\right)+\theta \theta\left(\mathcal{G}+\mathcal{G}^{\prime}\right)+\cdots
\end{aligned}
$$

The partially twisted action now follows from the known result for coupling a six-dimensional hypermultiplet to a background gauge field in flat space presented in [41]. Indeed, because manifest off-shell supersymmetry is preserved at all stages, for our purposes it is enough to repackage this result in the partially twisted theory. In $\mathcal{N}=1$ superspace the action is

$$
\begin{aligned}
I_{\Sigma} & =\int_{\mathbb{R}^{3,1} \times \Sigma} d^{4} x d^{4} \theta \omega \wedge\left[\left(\boldsymbol{\Lambda}, e^{-2 V-2 V^{\prime}} \boldsymbol{\Lambda}\right)+\left(\boldsymbol{\Lambda}^{c}, e^{2 V+2 V^{\prime}} \boldsymbol{\Lambda}^{c}\right)\right] \\
& +\int_{\mathbb{R}^{3,1} \times \Sigma} d^{4} x d^{2} \theta\left\langle\boldsymbol{\Lambda}^{c}, \bar{\partial}_{\mathbf{A}+\mathbf{A}^{\prime}} \boldsymbol{\Lambda}\right\rangle+\text { h.c. }
\end{aligned}
$$

In order to define the D-terms appearing in (D.8), we introduce a hermitian metric $(\cdot, \cdot)$ on the bundle $K_{\Sigma}^{1 / 2} \otimes \mathscr{U} \otimes \mathscr{U}^{\prime}$ in which the chiral superfield $\boldsymbol{\Lambda}$ is valued, and similarly for the bundle $K_{\Sigma}^{1 / 2} \otimes \mathscr{U}^{*} \otimes\left(\mathscr{U}^{\prime}\right)^{*}$ in which $\Lambda^{c}$ is valued. If $h_{\bar{a} a}$ represents the hermitian metric on $K_{\Sigma}^{1 / 2} \otimes \mathscr{U} \otimes \mathscr{U}^{\prime}$ in a local basis, then

$$
\left(\boldsymbol{\Lambda}, e^{-2 V-2 V^{\prime}} \boldsymbol{\Lambda}\right)=h_{\bar{a} a} \overline{\Lambda^{a}} e^{-2 V-2 V^{\prime}} \Lambda^{a}
$$

We emphasize that the pairing $(\cdot, \cdot)$ includes an implicit complex conjugation on one argument, as apparent above. Because the quantity in (D.10) transforms as a scalar on $\Sigma$, we use the pullback of the Kähler form $\omega$ on $S$ to define an integration measure over $\Sigma$ in (D.8).

In contrast to (D.8), the expression in (D.9) is defined without reference to a metric on either the bundles $\mathscr{U}$ and $\mathscr{U}^{\prime}$ or on $\Sigma$. Thus $\langle\cdot, \cdot\rangle$ indicates the canonical dual pairing between $\mathscr{U} \otimes \mathscr{U}^{\prime}$ and $\mathscr{U}^{*} \otimes\left(\mathscr{U}^{\prime}\right)^{*}$, so that $\left\langle\boldsymbol{\Lambda}^{c}, \bar{\partial}_{\mathbf{A}+\mathbf{A}^{\prime}} \boldsymbol{\Lambda}\right\rangle$ transforms as 
a differential form of type $(1,1)$ appropriate to integrate over $\Sigma$. Indeed, this is an expected consequence of twisting the field content of the six-dimensional theory.

Whereas the bulk gauge fields $\mathbf{A}$ and $\mathbf{A}^{\prime}$ naturally couple to the six-dimensional fields via the superpotential in (D.9), a natural superpotential coupling involving $\boldsymbol{\Phi}$ does not appear to exist, since $\boldsymbol{\Phi}$ transforms as a $(2,0)$-form on $S$.

To proceed further, we next expand $I_{\Sigma}$ in terms of component fields. This yields

$$
\begin{gathered}
I_{\Sigma}=\int_{\mathbb{R}^{3,1} \times \Sigma} d^{4} x \omega \wedge\left[(\mathcal{K}, \mathcal{K})+\left(\mathcal{K}^{c}, \mathcal{K}^{c}\right)-\left(D_{\mu} \sigma, D^{\mu} \sigma\right)-\left(D_{\mu} \sigma^{c}, D^{\mu} \sigma^{c}\right)\right. \\
\left.-\left(\sigma,\left(\mathcal{D}+\mathcal{D}^{\prime}\right) \cdot \sigma\right)+\left(\sigma^{c},\left(\mathcal{D}+\mathcal{D}^{\prime}\right) \cdot \sigma^{c}\right)\right]+ \\
+\int_{\mathbb{R}^{3,1} \times \Sigma} d^{4} x\left[\left\langle\mathcal{K}^{c}, \bar{\partial}_{A+A^{\prime}} \sigma\right\rangle+\left\langle\sigma^{c}, \bar{\partial}_{A+A^{\prime}} \mathcal{K}\right\rangle+\left\langle\sigma^{c},\left(\mathcal{G}+\mathcal{G}^{\prime}\right) \cdot \sigma\right\rangle\right. \\
\left.+\left\langle\overline{\mathcal{K}}^{c}, \partial_{A+A^{\prime}} \bar{\sigma}\right\rangle+\left\langle\bar{\sigma}^{c}, \partial_{A+A^{\prime}} \overline{\mathcal{K}}\right\rangle+\left\langle\bar{\sigma}^{c},\left(\overline{\mathcal{G}}+\overline{\mathcal{G}}^{\prime}\right) \cdot \bar{\sigma}\right\rangle\right]+\cdots
\end{gathered}
$$

where the "..." indicate additional fermionic terms in $I_{\Sigma}$. Also, in expressions such as $\mathcal{D} \cdot \sigma$, we indicate the action of elements in the Lie algebra of the group $G_{S}$ on the representation $U$, and similarly for $G_{S^{\prime}}$ and $U^{\prime}$.

We now determine both the on-shell supersymmetry transformations for the sixand eight-dimensional fields as well as the resulting BPS equations. Integrating out the six-dimensional auxiliary fields yields the conditions

$$
\begin{array}{ll}
\overline{\mathcal{K}}=\star_{\Sigma} \partial_{A+A^{\prime}} \bar{\sigma}^{c}, & \overline{\mathcal{K}^{c}}=-\star_{\Sigma} \partial_{A+A^{\prime}} \bar{\sigma}, \\
\mathcal{K}=\star_{\Sigma} \bar{\partial}_{A+A^{\prime}} \sigma^{c}, & \mathcal{K}^{c}=-\star_{\Sigma} \bar{\partial}_{A+A^{\prime}} \sigma .
\end{array}
$$

Here $\star_{\Sigma}$ denotes the duality operator acting on sections of $K_{\Sigma}^{1 / 2} \otimes \mathscr{U} \otimes \mathscr{U}^{\prime}$ and $K_{\Sigma}^{1 / 2} \otimes \mathscr{U}^{*} \otimes\left(\mathscr{U}^{\prime}\right)^{*}$ as determined by the given metrics on $\Sigma$ and $\mathscr{U} \otimes \mathscr{U}^{\prime}$.

Whereas the supersymmetry transformations for the six-dimensional bosons such as $\sigma$ are the same both on and off-shell, the on-shell supersymmetry transformations of the fermions follow by substituting in the values of the auxiliary fields in the 
off-shell transformations of (D.2) so that:

$$
\begin{array}{rlrl}
\delta_{\alpha} \lambda_{\beta} & =-\sqrt{2} \epsilon_{\alpha \beta} \star_{\Sigma} \bar{\partial}_{A+A^{\prime}} \sigma^{c}, & & \bar{\delta}_{\dot{\alpha}} \lambda_{\beta}=i \sqrt{2}\left(\sigma^{\mu}\right)_{\beta \dot{\alpha}} D_{\mu} \sigma, \\
\delta_{\alpha} \bar{\lambda}_{\dot{\beta}}=i \sqrt{2}\left(\sigma^{\mu}\right)_{\alpha \dot{\beta}} D_{\mu} \bar{\sigma}, & \bar{\delta}_{\dot{\alpha}} \bar{\lambda}_{\dot{\beta}}=-\sqrt{2} \epsilon_{\dot{\alpha} \dot{\beta}} \star_{\Sigma} \partial_{A+A^{\prime}} \bar{\sigma}^{c}, \\
\delta_{\alpha} \lambda_{\beta}^{c}=\sqrt{2} \epsilon_{\alpha \beta} \star_{\Sigma} \bar{\partial}_{A+A^{\prime}} \sigma, & & \bar{\delta}_{\dot{\alpha}} \lambda_{\beta}^{c}=i \sqrt{2}\left(\sigma^{\mu}\right)_{\beta \dot{\alpha}} D_{\mu} \sigma^{c}, \\
\delta_{\alpha} \bar{\lambda}_{\dot{\beta}}=i \sqrt{2}\left(\sigma^{\mu}\right)_{\alpha \dot{\beta}} D_{\mu} \overline{\sigma^{c}}, & \bar{\delta}_{\dot{\alpha}} \bar{\lambda}_{\dot{\beta}}^{c}=\sqrt{2} \epsilon_{\dot{\alpha} \dot{\beta}} \star_{\Sigma} \partial_{A+A^{\prime}} \bar{\sigma} .
\end{array}
$$

Some of the on-shell supersymmetry transformations of the eight-dimensional fields also change in the presence of the six-dimensional defect theory. Integrating out the auxiliary fields $\mathcal{G}$ and $\overline{\mathcal{G}}$ from the total action $I=I_{S}+I_{\Sigma}$ implies

$$
\begin{aligned}
2 i \omega \wedge \overline{\mathcal{G}} & =\bar{\partial}_{A} \varphi-\delta_{\Sigma}\left\langle\left\langle\sigma^{c}, \sigma\right\rangle\right\rangle_{\operatorname{ad}(P)}, \\
-2 i \omega \wedge \mathcal{G} & =\partial_{A} \bar{\varphi}-\delta_{\Sigma}\left\langle\left\langle\bar{\sigma}^{c}, \bar{\sigma}\right\rangle\right\rangle_{\operatorname{ad}(P)} .
\end{aligned}
$$

Here we use $\langle\langle\cdot, \cdot\rangle\rangle_{\mathrm{ad}(P)}$ to denote the canonical 'outer-product' determined by the action of $G$ on the representation $U$,

$$
\langle\langle\cdot, \cdot\rangle\rangle_{\operatorname{ad}(P)}:\left.\left[\mathscr{U} \otimes \mathscr{U}^{\prime}\right] \otimes\left[\mathscr{U}^{*} \otimes\left(\mathscr{U}^{\prime}\right)^{*}\right] \longrightarrow \operatorname{ad}(P)\right|_{\Sigma} .
$$

Explicitly, if $\left(T^{I}\right)_{a^{\prime}}^{a}$ for $I=1, \ldots, \operatorname{dim}\left(G_{S}\right)$ represent the generators of $G_{S}$ acting on $U$ in a given basis, then $\left\langle\left\langle\sigma^{c}, \sigma\right\rangle\right\rangle_{\mathrm{ad}(P)}=\sigma_{a}^{c}\left(T^{I}\right)_{a^{\prime}}^{a} \sigma^{a^{\prime}}$. Also, $\delta_{\Sigma}$ is a two-form with delta-function support which represents the Poincaré dual of the holomorphic curve $\Sigma$ inside $S$.

Based upon (D.18), the on-shell variations of the eight-dimensional fermions $\psi_{\alpha} \bar{m}$ and $\bar{\psi}_{\dot{\alpha} m}$ are now

$$
\begin{aligned}
& \delta_{\alpha} \psi_{\beta}=\sqrt{2} \epsilon_{\alpha \beta}\left(\bar{\partial}_{A}^{\dagger} \bar{\varphi}+\star_{S} \delta_{\Sigma}\left\langle\left\langle\sigma^{c}, \sigma\right\rangle\right\rangle_{\mathrm{ad}(P)}\right), \quad \bar{\delta}_{\dot{\alpha}} \psi_{\beta}=i \sqrt{2}\left(\sigma^{\mu}\right)_{\beta \dot{\alpha}} F_{\mu S}^{(0,1)} \\
& \bar{\delta}_{\dot{\alpha}} \bar{\psi}_{\dot{\beta}}=\sqrt{2} \epsilon_{\dot{\alpha} \dot{\beta}}\left(\partial_{A}^{\dagger} \varphi+\star_{S} \delta_{\Sigma}\left\langle\left\langle\bar{\sigma}^{c}, \bar{\sigma}\right\rangle\right\rangle_{\mathrm{ad}(P)}\right), \quad \delta_{\alpha} \bar{\psi}_{\dot{\beta} m}=i \sqrt{2}\left(\sigma^{\mu}\right)_{\alpha \dot{\beta}} F_{\mu S}^{(1,0)}
\end{aligned}
$$

Similarly, by integrating out the eight-dimensional auxiliary field $\mathcal{D}$ from the total action $I=I_{S}+I_{\Sigma}$, we arrive at the modified relation

$$
\mathcal{D}=-\star_{S}\left(\omega \wedge\left[F_{S}^{(1,1)}-\frac{1}{2} \delta_{\Sigma} \mu(\bar{\sigma}, \sigma)+\frac{1}{2} \delta_{\Sigma} \mu\left(\bar{\sigma}^{c}, \sigma^{c}\right)\right]+\frac{i}{2}[\varphi, \bar{\varphi}]\right) .
$$


Here $\mu(\bar{\sigma}, \sigma)$ denotes the moment map associated to the action of $G_{S}$ on the representation $U$, and similarly for $\mu\left(\bar{\sigma}^{c}, \sigma^{c}\right)$. In terms of the local generators $\left(T^{I}\right)_{a^{\prime}}^{a}$ which we introduced to describe the outer-product $\langle\langle\cdot, \cdot\rangle\rangle_{\mathrm{ad}(P)}$, the moment map is given as usual by $\mu(\bar{\sigma}, \sigma)=\bar{\sigma}^{\bar{a}}\left(T^{I}\right)_{\bar{a} a} \sigma^{a}$. The on-shell supersymmetry transformations for the eight-dimensional zero-form fermions are then given by (B.2) but now with $\mathcal{D}$ as in equation (D.21),

$$
\begin{array}{rlrl}
\delta_{\alpha} \eta_{\beta} & =\left(\sigma^{\mu \nu}\right)_{\alpha \beta} F_{\mu \nu}-i \epsilon_{\alpha \beta} \mathcal{D}, & & \bar{\delta}_{\dot{\alpha}} \eta_{\beta}=0, \\
\delta_{\alpha} \bar{\eta}_{\dot{\beta}}=0, & \bar{\delta}_{\dot{\alpha} \bar{\eta}_{\dot{\beta}}}=\left(\bar{\sigma}^{\mu \nu}\right)_{\dot{\alpha} \dot{\beta}} F_{\mu \nu}+i \epsilon_{\dot{\alpha} \dot{\beta}} \mathcal{D} .
\end{array}
$$

Finally, by inspection of equation (D.11), we note that the auxiliary two-form fields $\mathcal{H}$ and $\overline{\mathcal{H}}$ do not couple to the fields of the six-dimensional theory. In particular, this implies that the on-shell supersymmetry transformations of the two-form fermions $\chi$ and $\bar{\chi}$ are unchanged in the presence of the six-dimensional defect.

The BPS equations of motion now follow from the requirement that the on-shell variation of all fermions must vanish in a supersymmetric vacuum. At a practical level, these are simply the D- and F-flat conditions which arise when all auxiliary fields have been set to zero. The BPS equations of motion for the six-dimensional fields $\sigma$ and $\sigma^{c}$ are therefore:

$$
\bar{\partial}_{A+A^{\prime}} \sigma=\bar{\partial}_{A+A^{\prime}} \sigma^{c}=0
$$

Because this derives from an F-term, it is protected from quantum corrections. The other BPS equation of motion which derives from an F-term corresponds to the equation of motion for $\varphi$ in the presence of the six-dimensional defect theory:

$$
\bar{\partial}_{A} \varphi=\delta_{\Sigma}\left\langle\left\langle\sigma^{c}, \sigma\right\rangle\right\rangle_{\mathrm{ad}(P)}, \quad \partial_{A} \bar{\varphi}=\left\langle\left\langle\bar{\sigma}^{c}, \bar{\sigma}\right\rangle\right\rangle_{\mathrm{ad}(P)} .
$$

Note that the above expressions are independent of any metric data. The final BPS equation of motion on $S$ which derives from an F-term is uncorrected by the presence of the six-dimensional defect because the auxiliary fields $\mathcal{H}$ and $\overline{\mathcal{H}}$ do not couple to the defect:

$$
F_{S}^{(2,0)}=0, \quad F_{S}^{(0,2)}=0 .
$$

Finally, the BPS equation of motion on $S$ which derives from a D-term is given 
by

$$
\omega \wedge F_{S}^{(1,1)}+\frac{i}{2}[\varphi, \bar{\varphi}]=\frac{1}{2} \omega \wedge \delta_{\Sigma}\left[\mu(\bar{\sigma}, \sigma)-\mu\left(\bar{\sigma}^{c}, \sigma^{c}\right)\right] .
$$

Due to the explicit dependence on the Kähler forms of $S$ and $\Sigma$, away from the regime of large volume for both $S$ and $\Sigma$, we expect that this equation will generally receive quantum corrections. Also, though for simplicity we have focused attention solely on the Yang-Mills theory which lives on the surface $S$, the parallel BPS equations hold for the eight-dimensional Yang-Mills theory on $S^{\prime}$.

\section{E A Vanishing Theorem}

In this appendix, we establish the vanishing theorem used in Section 3.3.2 to constrain the zero mode content of the bulk fields in the partially twisted Yang-Mills theory on $\mathbb{R}^{3,1} \times S$. See for instance the proof of Theorem 6.1 in [43] for another appearance of this vanishing theorem.

As throughout, we take $S$ to be a smooth, compact Kähler surface with Kähler form $\omega$ and canonical divisor $K_{S}$. In order to prove our vanishing theorem, we assume that $-K_{S} \geq 0$, in the sense that the anti-canonical divisor $-K_{S}$ is effective. We also assume that $h^{2,0}(S)=0$, as follows automatically if $-K_{S}$ happens to be ample. Examples of such surfaces include both the del Pezzo surfaces $d P_{n}$ and the Hirzebruch surfaces $\mathbb{F}_{n}$.

We now let $E$ be a complex vector bundle over $S$ which is endowed with a hermitian metric and a compatible unitary connection $A$ solving the hermitian YangMills equations on $S$,

$$
F_{A}^{(2,0)}=F_{A}^{(0,2)}=\omega \cdot F_{A}=0 .
$$

Here $\omega \cdot F_{A}$ is shorthand for the contraction of $\omega$ with $F_{A}$ as defined using the Kähler metric on $S$. In local coordinates $\left(s^{n}, \bar{s}^{\bar{n}}\right)$ on $S$,

$$
\omega \cdot F_{A}=\omega^{n \bar{n}}\left[F_{A}\right]_{n \bar{n}}
$$

where we sum over repeated indices $n, \bar{n}=1,2$ in (E.2).

Because $\omega$ is self-dual, the vanishing of $\omega \cdot F_{A}$ is equivalent to the vanishing of $\omega \wedge F_{A}$. Hence the equations in (E.1) correspond to the bulk BPS equations (3.32), (3.33), (3.34) on $S$ in a background with $\varphi=0$, where $E$ is any vector bundle asso- 
ciated to the principal $G$-bundle $P$ on $S$. In fact, if $S$ is a del Pezzo or Hirzebruch surface, $\varphi$ necessarily vanishes for any solution of the bulk BPS equations. Otherwise, if $\varphi$ were nontrivial as an element in $H \frac{0}{\partial}\left(S, K_{S} \otimes \operatorname{ad}(P)\right)$, then at least one Casimir of $\varphi$ would be non-vanishing. Hence some positive power of $K_{S}$ would admit a non-trivial holomorphic section. But on del Pezzo and Hirzebruch surfaces, no positive powers of $K_{S}$ admit holomorphic sections, so $\varphi=0$.

We now state the vanishing theorem. First, we recall that because the $(2,0)$ and $(0,2)$ components of $F_{A}$ vanish according to (E.1), E admits a holomorphic structure. That is, if we let $d_{A}$ be the covariant derivative defined by the unitary connection on $E$, then the $(0,1)$ component $\bar{\partial}_{A}=d_{A}^{(0,1)}$ of the covariant derivative satisfies $\bar{\partial}_{A}^{2}=0$ and thereby defines a holomorphic structure on $E$.

Under the assumptions on $S$ above, we now claim that $E$ satisfies

$$
H \frac{2}{\partial}(S, E)=0 .
$$

Further, if $E$ is irreducible and non-trivial (meaning $E \neq \mathcal{O}_{S}$ ), then also

$$
H \frac{0}{\partial}(S, E)=0 .
$$

Proof of the Vanishing Theorem

To prove the vanishing theorem in (E.3), we first assume without loss that $E$ is irreducible. Otherwise, $E$ splits holomorphically as a direct sum of irreducible bundles $E_{j}$ for $j$ running in some index set $J$,

$$
E=\bigoplus_{j \in J} E_{j}
$$

Each summand $E_{j}$ also carries a unitary connection satisfying the hermitian YangMills equations in (E.1), and trivially

$$
H \frac{2}{\partial}(S, E)=\bigoplus_{j \in J} H \frac{2}{\partial}\left(S, E_{j}\right)
$$

Thus to demonstrate (E.3), we restrict attention to the irreducible summands of $E$.

The proof of the vanishing theorem now proceeds in two steps. In the first step, 
we show that if the irreducible bundle $E$ is non-trivial, then $H \frac{0}{\partial}(S, E)=0$. Alternatively, if $E$ is irreducible but trivial, so that $E=\mathcal{O}_{S}$, the vanishing theorem follows immediately by our assumption that $h^{2,0}(S)=0$. In the second step, assuming $E \neq \mathcal{O}_{S}$, we apply Serre duality to deduce the vanishing of $H_{\bar{\partial}}(S, E)$ from the vanishing of $H \frac{0}{\partial}(S, E)$.

To show that $H_{\bar{\partial}}(S, E)=0$ if $E$ is irreducible and non-trivial, let us assume otherwise. Therefore $E$ admits a non-trivial holomorphic section $s$.

We now recall a basic fact from Hodge theory. First, we let $d_{A}^{\dagger}$ and $\bar{\partial}_{A}^{\dagger}$ denote the adjoints of $d_{A}$ and $\bar{\partial}_{A}$ defined using the Kähler metric on $S$ and the hermitian metric on $E$. Then as shown for instance in Ch. 4 of [44], we have the following Hodge identity of linear operators acting on sections of $E$,

$$
2 \bar{\partial}_{A}^{\dagger} \bar{\partial}_{A}=d_{A}^{\dagger} d_{A}-i \omega \cdot F_{A}
$$

For completeness, we include a proof of (E.7) towards the end of this appendix. Because $\bar{\partial}_{A} s=\omega \cdot F_{A}=0$, we deduce from (E.7) that

$$
0=2 \int_{S}\left\|\bar{\partial}_{A} s\right\|^{2}=\int_{S}\left\|d_{A} s\right\|^{2} .
$$

Therefore $d_{A} s=0$, and $s$ is a covariantly constant section of $E$.

Because $s$ is covariantly constant, $s$ is a nowhere-vanishing holomorphic section of $E$. Hence $s$ defines an inclusion of the trivial line bundle $\mathcal{O}_{S}$ as a rank one holomorphic subbundle of $E$,

$$
0 \longrightarrow \mathcal{O}_{S} \stackrel{s}{\longrightarrow} E
$$

We let $\mathcal{O} \frac{\perp}{S}$ denote the orthocomplement to $s$ in $E$, defined using the hermitian metric on $E$. Because $d_{A}$ respects that metric, $\mathcal{O}_{S}^{\perp}$ in turn carries an induced unitary connection $^{23}$ satisfying the hermitian Yang-Mills equations (E.1), and in particular $\mathcal{O} \frac{\perp}{S}$ is holomorphic. Consequently $E$ splits as a sum of holomorphic bundles

$$
E=\mathcal{O}_{S} \oplus \mathcal{O}_{S}^{\perp}
$$

\footnotetext{
${ }^{23}$ Note that if $s^{\perp}$ is any section of $\mathcal{O} \frac{\perp}{S}$, then $0=d\left(s, s^{\perp}\right)=\left(d_{A} s, s^{\perp}\right)+\left(s, d_{A} s^{\perp}\right)=\left(s, d_{A} s^{\perp}\right)$, so $d_{A}$ restricts immediately to a unitary connection on $\mathcal{O} \frac{\perp}{S}$.
} 
Since $E \neq \mathcal{O}_{S}$, the bundle $\mathcal{O}_{S}^{\perp}$ must have non-zero rank, but then (E.10) contradicts our assumption that $E$ be irreducible. Hence

$$
H \frac{0}{\partial}(S, E)=0 .
$$

We are left to consider (E.11) in light of Serre duality. Under duality,

$$
H \frac{2}{\partial}(S, E) \cong H \frac{0}{\partial}\left(S, E^{*} \otimes \mathcal{O}_{S}\left(K_{S}\right)\right)^{*} .
$$

However, because $-K_{S} \geq 0$ is effective, we also have an inclusion

$$
H \frac{0}{\partial}\left(S, E^{*} \otimes \mathcal{O}_{S}\left(K_{S}\right)\right) \subseteq H \frac{0}{\partial}\left(S, E^{*}\right) .
$$

The inclusion in (E.13) follows once we recall that sections of $\mathcal{O}_{S}\left(K_{S}\right)$ for $K_{S} \leq 0$ can be interpreted as holomorphic functions on $S$ which vanish along the effective divisor $-K_{S}$. As a result, holomorphic sections of the product $E^{*} \otimes \mathcal{O}_{S}\left(K_{S}\right)$ are the same as holomorphic sections of $E^{*}$ which similarly vanish along $-K_{S}$.

Now, if $E$ admits a non-trivial irreducible unitary connection satisfying the hermitian Yang-Mills equations (E.1), then so does the dual $E^{*}$. Thus the vanishing result in (E.11) applies equally well to $E^{*}$,

$$
H \frac{0}{\partial}\left(S, E^{*}\right)=0 .
$$

Together, (E.12), (E.13), and (E.14) finally imply the basic vanishing theorem

$$
H \frac{2}{\partial}(S, E)=0 .
$$

\section{Corollaries}

The vanishing theorem in (E.15) has a few immediate corollaries which strongly constrain the low-energy spectrum of the partially twisted Yang-Mills theory on $\mathbb{R}^{3,1} \times S$. First, as we observed in relation to (E.14), if $E$ admits a hermitian Yang-

Mills connection satisfying (E.1), then so does $E^{*}$. Thus the vanishing theorem for $E$ immediately applies to $E^{*}$ as well, so that

$$
H \frac{2}{\partial}\left(S, E^{*}\right)=0 .
$$


Equivalently by Serre duality,

$$
H \frac{0}{\partial}\left(S, E \otimes \mathcal{O}\left(K_{S}\right)\right)=0
$$

More generally, since $E$ is any vector bundle associated to the principal $G$-bundle $P$ on $S$, the vanishing theorem trivially applies to any complex vector bundle $\rho(E)$ constructed by taking tensor products of $E$ and $E^{*}$, such as $\wedge^{2} E$ or $\operatorname{End}_{0}(E)$. Equivalently, the hermitian Yang-Mills connection on $E$ induces a corresponding connection on $\rho(E)$. Thus the vanishing theorem in (E.15) can be recast more broadly as

$$
H \frac{2}{\partial}(S, \rho(E))=0
$$

Finally, the bulk Yukawa couplings (3.54) on $S$ always involve the appearance of $H \frac{2}{\partial}(S, E)^{*}$ for some vector bundle $E$ associated to the principal $G$-bundle $P$ on $S$. Hence besides constraining the massless spectrum, the vanishing theorem (E.15) implies that all bulk Yukawa couplings vanish when the Kähler surface $S$ satisfies $-K_{S} \geq 0$ and $h^{2,0}(S)=0$.

\section{A Hodge Identity}

For the sake of completeness, we include here a proof of the Hodge identity in (E.7). This identity holds for sections of an arbitrary holomorphic vector bundle $E$ over a compact Kähler manifold $S$.

To setup notation, let $\Lambda$ be the operator which acts on sections of $\Omega_{S}^{*} \otimes E$ by contraction with the Kähler form $\omega$. According to standard Hodge theory (see $\S 0.7$ and $\S 1.2$ of $[45]), \Lambda$ satisfies

$$
\bar{\partial}_{A}^{\dagger}=i\left[\partial_{A}, \Lambda\right], \quad \partial_{A}^{\dagger}=-i\left[\bar{\partial}_{A}, \Lambda\right]
$$

Here $\partial_{A}$ and $\bar{\partial}_{A}$ are the $(1,0)$ and $(0,1)$ components of the covariant derivative $d_{A}$ acting on sections of $\Omega_{S}^{*} \otimes E$,

According to (E.19), when $\bar{\partial}^{\dagger}$ and $\partial_{A}^{\dagger}$ act on bundle-valued forms of type $(0,1)$ or $(1,0)$ (which are trivially annihilated by $\Lambda$ ), we can write

$$
\bar{\partial}_{A}^{\dagger}=-i \Lambda \partial_{A}, \quad \partial_{A}^{\dagger}=i \Lambda \bar{\partial}_{A}
$$


Thus, when acting on sections of $E$,

$$
\begin{aligned}
d_{A}^{\dagger} d_{A} & =\left(\partial_{A}^{\dagger}+\bar{\partial}_{A}^{\dagger}\right)\left(\partial_{A}+\bar{\partial}_{A}\right) \\
& =\left(i \Lambda \bar{\partial}_{A}-i \Lambda \partial_{A}\right)\left(\partial_{A}+\bar{\partial}_{A}\right) \\
& =-i \Lambda\left(\partial_{A} \bar{\partial}_{A}-\bar{\partial}_{A} \partial_{A}\right)
\end{aligned}
$$

In passing to the final line of (E.21), we use that $\partial_{A}^{2}=\bar{\partial}_{A}^{2}=0$.

For the same reason, $F_{A}=d_{A}^{2}=\partial_{A} \bar{\partial}_{A}+\bar{\partial}_{A} \partial_{A}$. So again when acting on sections of $E$,

$$
\omega \cdot F_{A}=\Lambda d_{A}^{2}=\Lambda\left(\partial_{A} \bar{\partial}_{A}+\bar{\partial}_{A} \partial_{A}\right) .
$$

Comparing (E.21) to (E.22) and using the identity in (E.20), we conclude

$$
\begin{aligned}
d_{A}^{\dagger} d_{A} & =i \omega \cdot F_{A}-2 i \Lambda \partial_{A} \bar{\partial}_{A}, \\
& =i \omega \cdot F_{A}+2 \bar{\partial}_{A}^{\dagger} \bar{\partial}_{A} .
\end{aligned}
$$

Examples for Line Bundles on $S$

We now describe some examples of line bundles on $S$ which admit an anti-self-dual connection satisfying the Hermitian Yang-Mills equations in (E.1). Although we do not present an exhaustive list, there are a large number of candidate line bundles which allow us to tune the chiral matter spectrum of the resulting theory. As a warmup, we first characterize all candidate line bundles for the Hirzebruch surfaces $\mathbb{F}_{n}$ for $n \geq 0$. A candidate Kähler class $\omega=a f+b \sigma$ satisfies:

$$
\omega \cdot f, \omega \cdot \sigma>0
$$

or:

$$
b>0, a>b n .
$$

Given a line bundle $L$ on $\mathbb{F}_{n}$ such that $c_{1}(L)=A f+B \sigma$, the condition that $c_{1}(L)$ be anti-self-dual implies

$$
\omega \cdot c_{1}(L)=A b+B(a-b n)=0
$$

which admits a non-trivial solution for $a$ and $b$ satisfying the inequalities of (E.25) 
if and only if $A B<0$.

We now consider line bundles $L$ on a del Pezzo surface $d P_{n}$ for which $c_{1}(L)$ is given by

$$
c_{1}(L)=\sum_{i=1}^{n} a_{i} E_{i} .
$$

We claim that if $a_{i} a_{j}<0$ for some $i \neq j$, then there exists a parametric family of Kähler classes $\omega$ such that the condition $\omega \cdot c_{1}(L)=0$ holds.

Clearly, the condition on the integers $a_{i}$ implies that there exist positive integers $b_{i}>0$ such that

$$
\sum_{i=1}^{n} b_{i} a_{i}=0
$$

We next observe that the following choice for $\omega$ defines a Kähler class on $d P_{n}$ if the parameter $A$ appearing below is sufficiently large,

$$
\omega=A H-\sum_{i=1}^{n} b_{i} E_{i} .
$$

Indeed, by inspection of the generators of the Kähler cone given in Appendix A, we note that when $A$ is sufficiently large,

$$
\omega \cdot G_{i}>0
$$

where $G_{i}$ is any effective divisor on $d P_{n}$. Finally, by construction, $\omega \cdot c_{1}(L)=0$.

We note that in the limit that $A$ is large, the volume of $S$ is also large. Although the D-term condition $\omega \cdot c_{1}(L)=0$ might receive quantum corrections away from the large-volume limit, we expect that the line bundles $L$ determined by (E.27) are still associated to supersymmetric solutions on $S$ when the volume of $S$ is large enough.

\section{F Explicit Deformations of an $E_{7}$ Singularity}

In this Appendix we collect the explicit expressions for the unfolding of the $E_{7}$ singularity used in subsection 4.3.2. The leading order behavior of each $\beta_{i}$ as a 
polynomial in the $t_{i}$ is:

$$
\begin{aligned}
& \beta_{2} \alpha^{2}=\alpha^{2}\left(16\left(s_{1}\right)^{2}+O\left(t_{i}\right)\right) \\
& \beta_{4} \alpha^{2}=\alpha^{2}\left(\frac{16}{3}\left(s_{2}\right)^{2}-8 s_{1} s_{3}+O\left(t_{i}\right)\right) \\
& \beta_{6} \alpha^{3}=\alpha^{3}\left(-\frac{128}{27}\left(s_{2}\right)^{3}+\frac{32}{3} s_{1} s_{2} s_{3}-\frac{32}{3}\left(s_{1}\right)^{2} s_{4}+O\left(t_{i}^{2}\right)\right) \\
& \beta_{8} \alpha^{3}=\alpha^{3}\left(\begin{array}{l}
-\frac{8}{3} s_{2}\left(s_{3}\right)^{2}+\frac{32}{9}\left(s_{2}\right)^{2} s_{4}+\frac{8}{3} s_{1} s_{3} s_{4}-\frac{16}{3} s_{1} s_{2} s_{5} \\
+16\left(s_{1}\right)^{2} s_{6}+O\left(t_{i}^{2}\right)
\end{array}\right) \\
& \beta_{10} \alpha^{4}=\alpha^{4}\left(\begin{array}{l}
\frac{32}{9}\left(s_{2}\right)^{2}\left(s_{3}\right)^{2}-\frac{8}{3} s_{1}\left(s_{3}\right)^{3}-\frac{128}{27}\left(s_{2}\right)^{3} s_{4}-\frac{16}{3}\left(s_{1}\right)^{2}\left(s_{4}\right)^{2} \\
+\frac{64}{9} s_{1}\left(s_{2}\right)^{2} s_{5}+\frac{32}{3}\left(s_{1}\right)^{2} s_{3} s_{5}+\frac{32}{3}\left(s_{1}\right)^{2} s_{2} s_{6} \\
-64\left(s_{1}\right)^{3} s_{7}+O\left(t_{i}^{3}\right)
\end{array}\right) \\
& \beta_{12} \alpha^{4}=\alpha^{4}\left(\begin{array}{l}
\frac{1}{3}\left(s_{3}\right)^{4}-\frac{8}{9} s_{2}\left(s_{3}\right)^{2} s_{4}+\frac{16}{27}\left(s_{2}\right)^{2}\left(s_{4}\right)^{2}+\frac{16}{9} s_{1} s_{3}\left(s_{4}\right)^{2} \\
-\frac{8}{3} s_{1}\left(s_{3}\right)^{2} s_{5}-\frac{16}{9} s_{1} s_{2} s_{4} s_{5}+\frac{16}{3}\left(s_{1}\right)^{2}\left(s_{5}\right)^{2}+8 s_{1} s_{2} s_{3} s_{6} \\
-\frac{32}{3}\left(s_{1}\right)^{2} s_{4} s_{6}-16 s_{1}\left(s_{2}\right)^{2} s_{7}+O\left(t_{i}^{3}\right)
\end{array}\right) \\
& \beta_{14} \alpha^{5}=\alpha^{5}\left(\begin{array}{l}
-\frac{8}{9} s_{2}\left(s_{3}\right)^{4}+\frac{64}{27}\left(s_{2}\right)^{2}\left(s_{3}\right)^{2} s_{4}+\frac{8}{9} s_{1}\left(s_{3}\right)^{3} s_{4} \\
-\frac{128}{81}\left(s_{2}\right)^{3}\left(s_{4}\right)^{2}-\frac{128}{81}\left(s_{2}\right)^{3}\left(s_{4}\right)^{2}-\frac{32}{9} s_{1} s_{2} s_{3}\left(s_{4}\right)^{2} \\
+\frac{64}{27}\left(s_{1}\right)^{2}\left(s_{4}\right)^{3}+\frac{16}{9} s_{1} s_{2}\left(s_{3}\right)^{2} s_{5}+\frac{128}{27} s_{1}\left(s_{2}\right)^{2} s_{4} s_{5} \\
-\frac{32}{9}\left(s_{1}\right)^{2} s_{3} s_{4} s_{5}+\frac{64}{9}\left(s_{1}\right)^{2} s_{2}\left(s_{5}\right)^{2}-\frac{32}{3} s_{1}\left(s_{2}\right)^{2} s_{3} s_{6} \\
+\frac{16}{3}\left(s_{1}\right)^{2}\left(s_{3}\right)^{2} s_{6}-\frac{32}{9}\left(s_{1}\right)^{2} s_{2} s_{4} s_{6}-\frac{64}{3}\left(s_{1}\right)^{3} s_{5} s_{6} \\
+\frac{64}{3} s_{1}\left(s_{2}\right)^{3} s_{7}-64\left(s_{1}\right)^{2} s_{2} s_{3} s_{7}+\frac{256}{3}\left(s_{1}\right)^{3} s_{4} s_{7}+O\left(t_{i}^{4}\right)
\end{array}\right)
\end{aligned}
$$




$$
\beta_{18} \alpha^{6}=\alpha^{6}\left(\begin{array}{l}
\frac{2}{27}\left(s_{3}\right)^{6}-\frac{8}{27} s_{2}\left(s_{3}\right)^{4} s_{4}+\frac{32}{81}\left(s_{2}\right)^{2}\left(s_{3}\right)^{2}\left(s_{4}\right)^{2} \\
+\frac{16}{27} s_{1}\left(s_{3}\right)^{3}\left(s_{4}\right)^{2}-\frac{128}{729}\left(s_{2}\right)^{3}\left(s_{4}\right)^{3}-\frac{64}{81} s_{1} s_{2} s_{3}\left(s_{4}\right)^{3} \\
+\frac{64}{81}\left(s_{1}\right)^{2}\left(s_{4}\right)^{4}-\frac{8}{9} s_{1}\left(s_{3}\right)^{4} s_{5}+\frac{16}{27} s_{1} s_{2}\left(s_{3}\right)^{2} s_{4} s_{5} \\
+\frac{64}{81} s_{1}\left(s_{2}\right)^{2}\left(s_{4}\right)^{2} s_{5}-\frac{64}{27}\left(s_{1}\right)^{2} s_{3}\left(s_{4}\right)^{2} s_{5}+\frac{32}{9}\left(s_{1}\right)^{2}\left(s_{3}\right)^{2}\left(s_{5}\right)^{2} \\
+\frac{64}{27}\left(s_{1}\right)^{2} s_{2} s_{4}\left(s_{5}\right)^{2}-\frac{128}{27}\left(s_{1}\right)^{3}\left(s_{5}\right)^{3}+\frac{8}{3} s_{1} s_{2}\left(s_{3}\right)^{3} s_{6} \\
-\frac{32}{9} s_{1}\left(s_{2}\right)^{2} s_{3} s_{4} s_{6}-\frac{32}{9}\left(s_{1}\right)^{2}\left(s_{3}\right)^{2} s_{4} s_{6}-\frac{64}{27}\left(s_{1}\right)^{2} s_{2}\left(s_{4}\right)^{2} s_{6} \\
+\frac{32}{3}\left(s_{1}\right)^{2} s_{2} s_{3} s_{5} s_{6}+\frac{128}{9}\left(s_{1}\right)^{3} s_{4} s_{5} s_{6}+16\left(s_{1}\right)^{2}\left(s_{2}\right)^{2}\left(s_{6}\right)^{2} \\
-\frac{16}{3} s_{1}\left(s_{2}\right)^{2}\left(s_{3}\right)^{2} s_{7}+\frac{64}{9} s_{1}\left(s_{2}\right)^{3} s_{4} s_{7}+\frac{128}{3}\left(s_{1}\right)^{2} s_{2} s_{3} s_{4} s_{7} \\
-\frac{256}{9}\left(s_{1}\right)^{3}\left(s_{4}\right)^{2} s_{7}-\frac{128}{3}\left(s_{1}\right)^{2}\left(s_{2}\right)^{2} s_{5} s_{7}+O\left(t_{i}^{5}\right)
\end{array}\right) .
$$

By inspection, when a single $t_{i}$ develops a pole, we find that each coefficient is regular in $\alpha$ and does not vanish when $\alpha$ equals zero.

\section{G Higher Exotic Singularities}

The results of Section 5 provide a gauge theory interpretation of models where the singularity type $G_{S}$ of rank $r$ present at generic points of the surface enhances to a gauge group of rank $r+2$ at a discrete collection of points in $S$. Our expectation is that this analysis remains valid provided the singularity at such a point is of $A D E$ type. In this Appendix we discuss the more exotic possibility where the singularity type degenerates further. An example of this phenomenon is the minimal Weierstrass model:

$$
y^{2}=x^{3}+\alpha x z^{3}+\beta z^{5}+\gamma z^{6}+\gamma^{\prime} x z^{4} .
$$

so that $G_{\Sigma}=E_{8}$ and $G_{S}=E_{7}$. In a consistent compact model, the coefficients $\gamma$ and $\gamma^{\prime}$ control the separation between the singularity located at $z=0$ and other singularities at infinity in the $z$ coordinate.

At the intersection point of the curves $(\alpha=0)$ and $(\beta=0)$, the Weierstrass model degenerates further. This has been studied in detail in [23] where this singular behavior was matched to a codimension three degeneration of an instanton bundle in the corresponding heterotic dual. We first summarize the findings of [23] 
and also add a few points to its physical interpretation. As explained in [23], in the limit where the Kähler class of the elliptic fibration is of zero size, the resulting singularity cannot be resolved. Because a consistent F-theory compactification has vanishing Kähler class for the elliptic curve, we physically interpret this to mean that in a four-dimensional theory we can expect exotic physical phenomena from such enhancements in singularity type. However, as explained in [23], by performing a partial blowup of the $E_{7}$ singularity, the exotic singularity disappears. In F-theory this corresponds to the fact that if we compactify the theory on a circle to three dimensions and turn on a Wilson line around the circle, the exotic singularity disappears. In other words, no extra blowup is necessary to resolve the singularity. This suggests that whatever the degrees of freedom responsible for the exotic singularity may correspond to, these degrees of freedom are charged under the $E_{7}$ gauge group so that when an $E_{7}$ Wilson line develops a vev, the resulting degrees of freedom develop a mass.

Perhaps one of the most interesting aspects of the blowup considered in [23] is that the minimal resolution of the singularity breaks the $E_{7}$ group to a rank seven group which is given by $S U(3) \times S U(2) \times U(1) \times G$ for some rank three group $G$. This is remarkable because this breaking pattern is consistent with preserving the Standard Model gauge group! We find this quite intriguing and worthy of further investigation. Here we explain the relevant geometric facts about the blowup and explain how the Standard Model gauge group remains intact after the blowup.

To better understand the singular degeneration locus, we first study a blowup of the geometry near the singular locus $x=y=z=0$. Following [23], we introduce an additional projective coordinate $\lambda$ of weight -1 and perform a weighted blowup by assigning weights $1,2,3$ to $z, x$ and $y$. Performing the change of coordinates:

$$
x \mapsto \lambda^{2} x, y \mapsto \lambda^{3} y, z \mapsto \lambda z
$$

we note that this assignment of weights preserves the scaling of the holomorphic four form. The geometry is now given by:

$$
\lambda y^{2}=\lambda x^{3}+\alpha x z^{3}+\beta z^{5}+\lambda^{2} \gamma z^{6}+\lambda^{2} \gamma^{\prime} x z^{4}
$$

which reduces to the original Weierstrass model when $\lambda=1$. Note, however, that 
in the patch $\lambda=0$, the hypersurface becomes:

$$
0=\alpha x z^{3}+\beta z^{5}
$$

which is trivially satisfied when $\alpha=\beta=0$. Precisely at the point of degeneration, the parameters $x, y$ and $z$ are unconstrained and parameterize an entire weighted projective space $\mathbb{P}_{[1,2,3]}^{2}$. Note that the presence of the higher order terms $\gamma$ and $\gamma^{\prime}$ does not alter this conclusion so that the resulting physics remains insensitive to global details of the compactification. Away from points in $S$ where $\alpha$ and $\beta$ both vanish, the blowup corresponds to a curve inside of $\mathbb{P}_{[1,2,3]}^{2}$ which collapses to a point in the blowdown.

Although the blowup does not change the rank of the gauge group, the end result now contains additional simple group factors and a $U(1)$ factor at the location of the blown up node of the Dynkin diagram. We now show that the geometry still contains a $A_{2} \times A_{1}$ type singularity so that the non-abelian gauge group factors of the Standard Model remain intact. Restricting to the patch $x=1$ of equation (G.3) and dropping the irrelevant contribution from $\gamma$ and $\gamma^{\prime}$, note that rescaling the remaining coordinates by $\zeta$ yields:

$$
\zeta^{5} \lambda y^{2}=\zeta^{-1} \lambda+\zeta^{3} \alpha z^{3}+\zeta^{5} \beta z^{5}
$$

so that the above equation remains invariant when $\zeta^{2}=1$. Indeed, note that the condition $x=1$ is preserved under the rescaling $x \mapsto \zeta^{2} x$. This establishes the presence of an $A_{1}$ type singularity. Next restrict to the patch $y=1$. In this case, rescaling by $\eta$ yields:

$$
\eta^{-1} \lambda=\eta^{5} \lambda x^{3}+\eta^{5} \alpha x z^{3}+\eta^{5} \beta z^{5}
$$

so that the above equation remains invariant when $\eta^{6}=1$. On the other hand, the condition $y=1$ is preserved under the rescaling $y \mapsto \eta^{3} y$ provided $\eta^{3}$. This establishes the presence of a $\mathbb{Z}_{3} \times \mathbb{Z}_{2}=A_{2} \times A_{1}$ orbifold singularity in the geometry. 


\section{References}

[1] R. Blumenhagen, M. Cvetič, P. Langacker, and G. Shiu, "Toward Realistic Intersecting D-Brane Models," Ann. Rev. Nucl. Part. Sci. 55 (2005) 71-139, hep-th/0502005.

[2] R. Blumenhagen, B. Körs, D. Lüst, and S. Stieberger, "Four-dimensional String Compactifications with D-Branes, Orientifolds and Fluxes," Phys. Rept. 445 (2007) 1-193, hep-th/0610327.

[3] F. Marchesano, "Progress in D-brane model building," Fortsch. Phys. 55 (2007) 491-518, hep-th/0702094.

[4] D. Malyshev and H. Verlinde, "D-branes at Singularities and String Phenomenology," Nucl. Phys. B Proc. Suppl. 171 (2007) 139-163, arXiv:0711.2451 [hep-th].

[5] J. D. Lykken, E. Poppitz, and S. P. Trivedi, "Branes with GUTs and Supersymmetry Breaking," Nucl. Phys. B 543 (1999) 105-121, hep-th/9806080.

[6] Y. Achiman and B. Stech, "Quark Lepton Symmetry and Mass Scales in an E6 Unified Gauge Model," Phys. Lett. B 77 (1978) 389.

[7] F. Gursey and M. Serdaroglu, "E6 Gauge Field Theory Model Revisited," Nuovo Cim. A 65 (1981) 337.

[8] R. W. Robinett and J. L. Rosner, "Mass scales in grand unified theories," Phys. Rev. D 26 (1982) 2396-2419.

[9] D. Berenstein, "Branes vs GUTs: challenges for string inspired phenomenology," hep-th/0603103.

[10] J. J. Heckman, C. Vafa, H. Verlinde, and M. Wijnholt, "Cascading to the MSSM," arXiv:0711.0387 [hep-ph].

[11] D. J. Gross, J. A. Harvey, E. J. Martinec, and R. Rohm, "The Heterotic String," Phys. Rev. Lett. 54 (1985) 502-505. 
[12] E. Witten, "Strong coupling expansion of Calabi-Yau compactification," Nucl. Phys. B 471 (1996) 135-158, hep-th/9602070.

[13] P. Hořava and E. Witten, "Heterotic and Type I string dynamics from eleven dimensions," Nucl. Phys. B 460 (1996) 506-524, hep-th/9510209.

[14] F. Cachazo and C. Vafa, "Type I' and Real Algebraic Geometry," hep-th/0001029.

[15] B. Acharya and E. Witten, "Chiral Fermions from Manifolds Of $G_{2}$ Holonomy," hep-th/0109152.

[16] C. Vafa, "Evidence for F-theory," Nucl. Phys. B 469 (1996) 403-415, hep-th/9602022.

[17] M. Bershadsky, A. Johansen, T. Pantev, and V. Sadov, "On four-dimensional compactifications of F-theory," Nucl. Phys. B 505 (1997) 165-201, hep-th/9701165.

[18] B. Andreas and G. Curio, "On discrete twist and four-flux in $\mathrm{N}=1$ heterotic/F- theory compactifications," Adv. Theor. Math. Phys. 3 (1999) 1325-1413, hep-th/9908193.

[19] C. Beasley, J. J. Heckman, and C. Vafa work in progress.

[20] R. Donagi and M. Wijnholt, "Model Building with F-theory," arXiv:0802.2969 [hep-th].

[21] M. Bershadsky, K. Intriligator, S. Kachru, D. R. Morrison, V. Sadov, and C. Vafa, "Geometric singularities and enhanced gauge symmetries," Nucl. Phys. B 481 (1996) 215-252, hep-th/9605200.

[22] S. Katz and C. Vafa, "Matter from geometry," Nucl. Phys. B 497 (1997) 146-154, hep-th/9606086.

[23] P. Candelas, D. E. Diaconescu, B. Florea, D. R. Morrison, and G. Rajesh, "Codimension-three bundle singularities in F-theory," JHEP 06 (2002) 014, hep-th/0009228. 
[24] C. Vafa and E. Witten, "A Strong coupling test of S-duality," Nucl. Phys. B 431 (1994) 3-77, hep-th/9408074.

[25] E. Witten, "Non-Perturbative Superpotentials In String Theory," Nucl. Phys. B 474 (1996) 343-360, hep-th/9604030.

[26] D. R. Morrison and C. Vafa, "Compactifications of F-theory on Calabi-Yau threefolds (I)," Nucl. Phys. B 473 (1996) 74-92, hep-th/9602114.

[27] D. R. Morrison and C. Vafa, "Compactifications of F-theory on Calabi-Yau threefolds (II)," Nucl. Phys. B 476 (1996) 437-469, hep-th/9603161.

[28] S. Katz and D. R. Morrison, "Gorenstein Threefold Singularities with Small Resolutions via Invariant Theory for Weyl Groups," J. Alg. Geom. 1 (1992) 449-530, alg-geom/9202002.

[29] M. Bershadsky, C. Vafa, and V. Sadov, "D-branes and topological field theories," Nucl. Phys. B 463 (1996) 420-434, hep-th/9511222.

[30] R. Dijkgraaf and G. W. Moore, "Balanced Topological Field Theories," Commun. Math. Phys. 185 (1997) 411-440, hep-th/9608169.

[31] M. Bershadsky, K. Intriligator, S. Kachru, D. Morrison, V. Sadov, and C. Vafa, "Geometric Singularities and Enhanced Gauge Symmetries," Nucl. Phys. B481 (1996) 215-252, hep-th/9605200.

[32] N. R. Constable, J. Erdmenger, Z. Guralnik, and I. Kirsch, "Intersecting D3-branes and holography," Phys. Rev. D 68 (2003) 106007, hep-th/0211222.

[33] A. Kapustin and S. Sethi, "The Higgs Branch of Impurity Theories," Adv. Theor. Math. Phys. 2 (1998) 571-591, hep-th/9804027.

[34] O. DeWolfe, D. Z. Freedman, and H. Ooguri, "Holography and defect conformal field theories," Phys. Rev. D 66 (2002) 025009, hep-th/0111135.

[35] E. Witten, "Supersymmetric Yang-Mills Theory on a Four Manifold," J. Math. Phys. 35 (1994) 5101-5135, hep-th/9403195.

[36] S. Gukov and E. Witten, "Gauge Theory, Ramification, And The Geometric Langlands Program," hep-th/0612073. 
[37] J. Wess and J. Bagger, Supersymmetry and Supergravity. Princeton University Press, Princeton, New Jersey, 1992.

[38] L. Baulieu, H. Kanno, and I. M. Singer, "Special Quantum Field Theories in Eight and Other Dimensions," Commun. Math. Phys. 194 (1998) 149-175, hep-th/9704167.

[39] B. S. Acharya, J. M. Figueroa-O'Farrill, B. J. Spence, and M. O'Loughlin, "Euclidean D-branes and Higher-Dimensional Gauge Theory," Nucl. Phys. B514 (1998) 583-602, hep-th/9707118.

[40] N. Marcus, A. Sagnotti, and W. Siegel, "Ten-dimensional supersymmetric Yang-Mills theory in terms of four-dimensional superfields," Nucl. Phys. B 224 (1983) 159-179.

[41] N. Arkani-Hamed, T. Gregoire, and J. Wacker, "Higher dimensional supersymmetry in 4D superspace," JHEP 03 (2002) 055, hep-th/0101233.

[42] R. Dijkgraaf and C. Vafa, "N $\mathcal{N}=1$ Supersymmetry, Deconstruction and Bosonic Gauge Theories," hep-th/0302011.

[43] M. F. Atiyah, N. J. Hitchin, and I. M. Singer, "Self-Duality in Four-Dimensional Riemannian Geometry," Proc. R. Soc. Lond. A 362 (1978) 425-461.

[44] R. Friedman, Algebraic Surfaces and Holomorphic Vector Bundles. Springer-Verlag, New York, 1998.

[45] P. Griffiths and J. Harris, Principles of Algebraic Geometry. John Wiley \& Sons, Inc., New York, 1978. 